\title{
Low-Flow Characteristics of Streams in the Lower Wisconsin River Basin
}

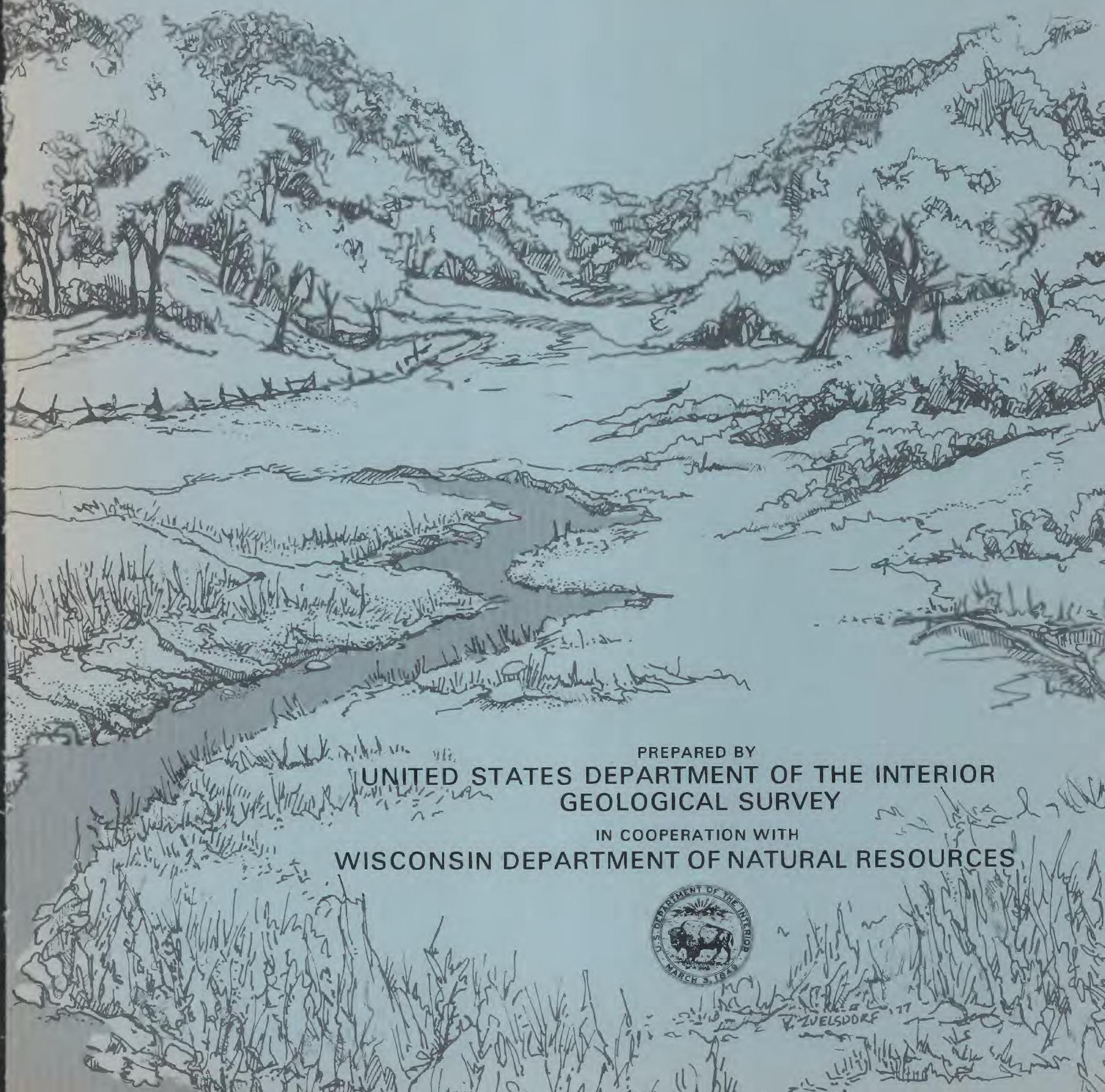




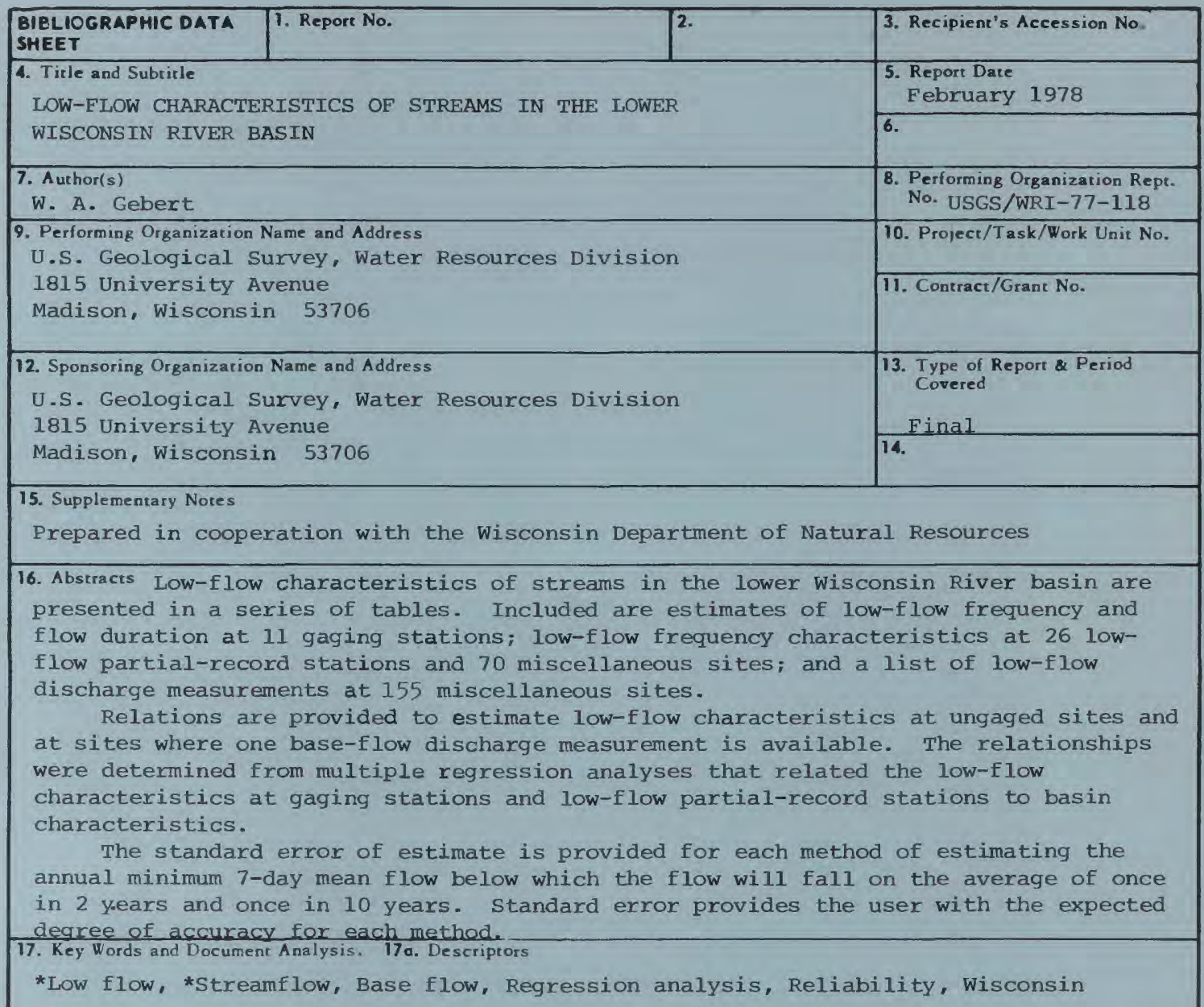

17b. Identifiers/Open-Ended Terms

7-day 10-year low flow, Flow duration, Low-flow frequency, Standard error of estimate, wisconsin River

17e. COSATI Field Group

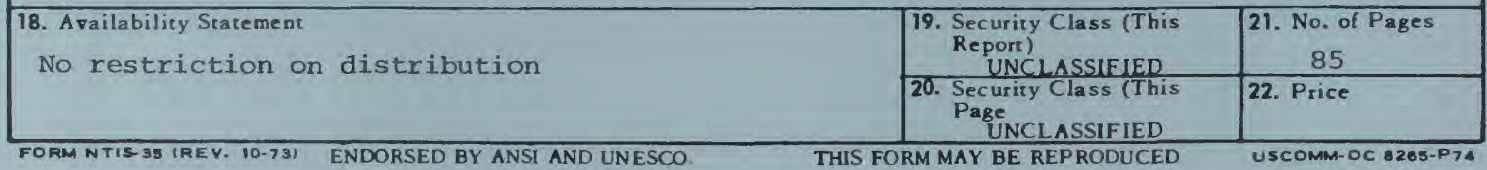




\title{
Low-flow Characteristics of Streams in the Lower Wisconsin River Basin
}

\author{
W. A. GEBERT
}

\section{U. S. GEOLOGICAL SURVEY}

Water Resources Investigations 77-118

Prepared in cooperation with the

Wisconsin Department of Natural Resources

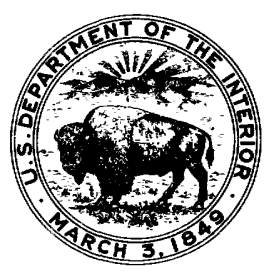

March 1978 


\title{
UNITED STATES DEPARTMENT OF THE INTERIOR
}

CECIL D. ANDRUS, SECRETARY

\section{GEOLOGICAL SURVEY}

\author{
W. A. Radlinski, Acting Director
}

For additional information write to:

U.S. Geological Survey

1815 University Avenue

Madison, Wisconsin 53706 


\section{CONTENTS}

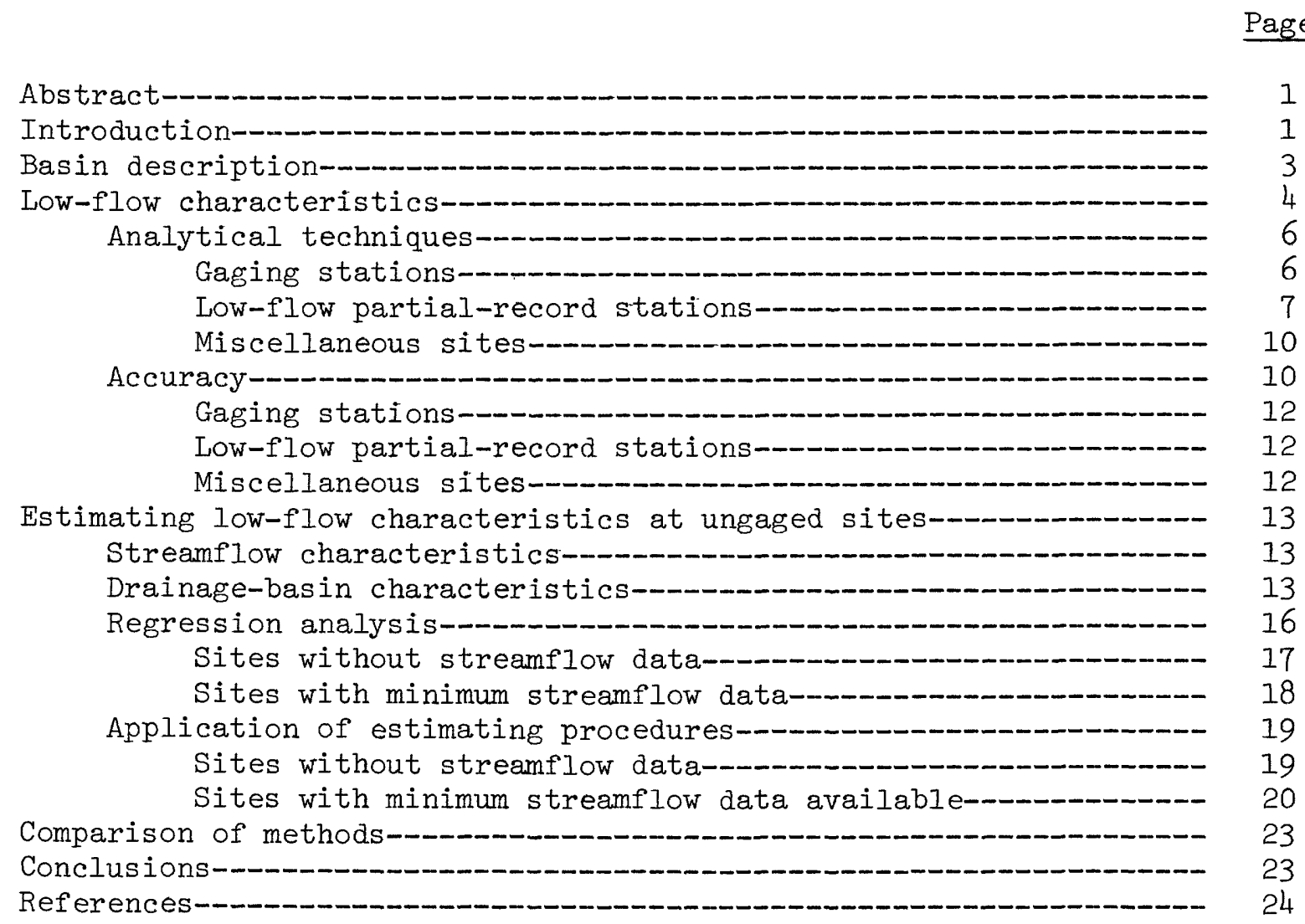

\section{ILLUSTRATIONS}

Plate 1. Location of gaging stations, low-flow partial-record stations, and miscellaneous sites

2. Base-flow index values for selected stations and sites

$\underline{\text { Page }}$

Figure 1. Map showing location of the lower Wisconsin River basin in Wisconsin-_-__-_-_-_ 2

2. Hydrograph of daily discharge of Black Earth Creek for 1966 climatic year, showing annual low-flow periods for various numbers of days-_._-_._-

3. Low-flow frequency curves showing magnitude and frequency of the annual minimum mean discharge for indicated number of consecutive days at Black Earth Creek 
Figure 4. Flow-duration curve showing percentage of time a given discharge was exceeded for Black Earth Creek-...-..-

5. Graph showing method of estimating Q7,2 and Q7,10 at low-flow partial-record stations from correlation of base-flow discharge measurements at Otter Creek with concurrent daily mean discharge of Black Earth Creek---

6. Graph showing method of estimating $Q 7,2$ and $Q 7,10$ at miscellaneous sites from correlation of base-flow discharge measurements at West Branch Honey Creek with concurrent daily mean discharge of Black Earth Creek

\section{TABLES}

Table 1. Low-flow characteristics for sites in the lower Wisconsin River basin-_-

2. Drainage-basin characteristics for low-flow partialrecord stations and selected gaging stations in the

3. Comparison of methods available to estimate low-flow characteristics in the lower Wisconsin River basin----- 


\title{
Low-flow Characteristics of Streams in the Lower Wisconsin River Basin
}

\author{
W. A. GEBERT
}

\begin{abstract}
Low-flow characteristics of streams in the lower Wisconsin River basin are presented. Included are estimates of low-flow frequency and flow duration at 11 gaging stations; low-flow frequency characteristics at 26 low-flow partial-record stations and 70 miscellaneous sites; and a list of low-flow discharge measurements at 155 miscellaneous sites where insufficient data were available to estimate low-flow characteristics.

Relations are provided to estimate low-flow characteristics at ungaged sites and at sites where one base-flow discharge measurement is available. The relationships were determined from multiple regression analyses that related the low-flow characteristics at gaging stations and low-flow partialrecord stations to basin characteristics.

The standard error of estimate is provided for each method of estimating the annual minimum 7-day mean flow below which the flow will fall on the average of once in 2 years and once in 10 years. Standard error provides the user with the expected degree of accuracy for each method.

Low-flow characteristics estimated for the lower Wisconsin River basin have a high degree of reliability when compared with other basins in Wisconsin. Reliable estimates appear to be related to the relatively uniform geologic features in the basin.
\end{abstract}

\section{INTRODUCTION}

The purpose of this report is to describe low-flow characteristics of streams in the lower Wisconsin River basin where streamflow data have been 
collected and to present equations for estimating low-flow characteristics at ungaged sites.

This study was done in cooperation with the Wisconsin Department of Natural Resources. This report is part of a series of twelve planned reports to describe low-flow characteristics of the major basins in Wisconsin (fig. 1).

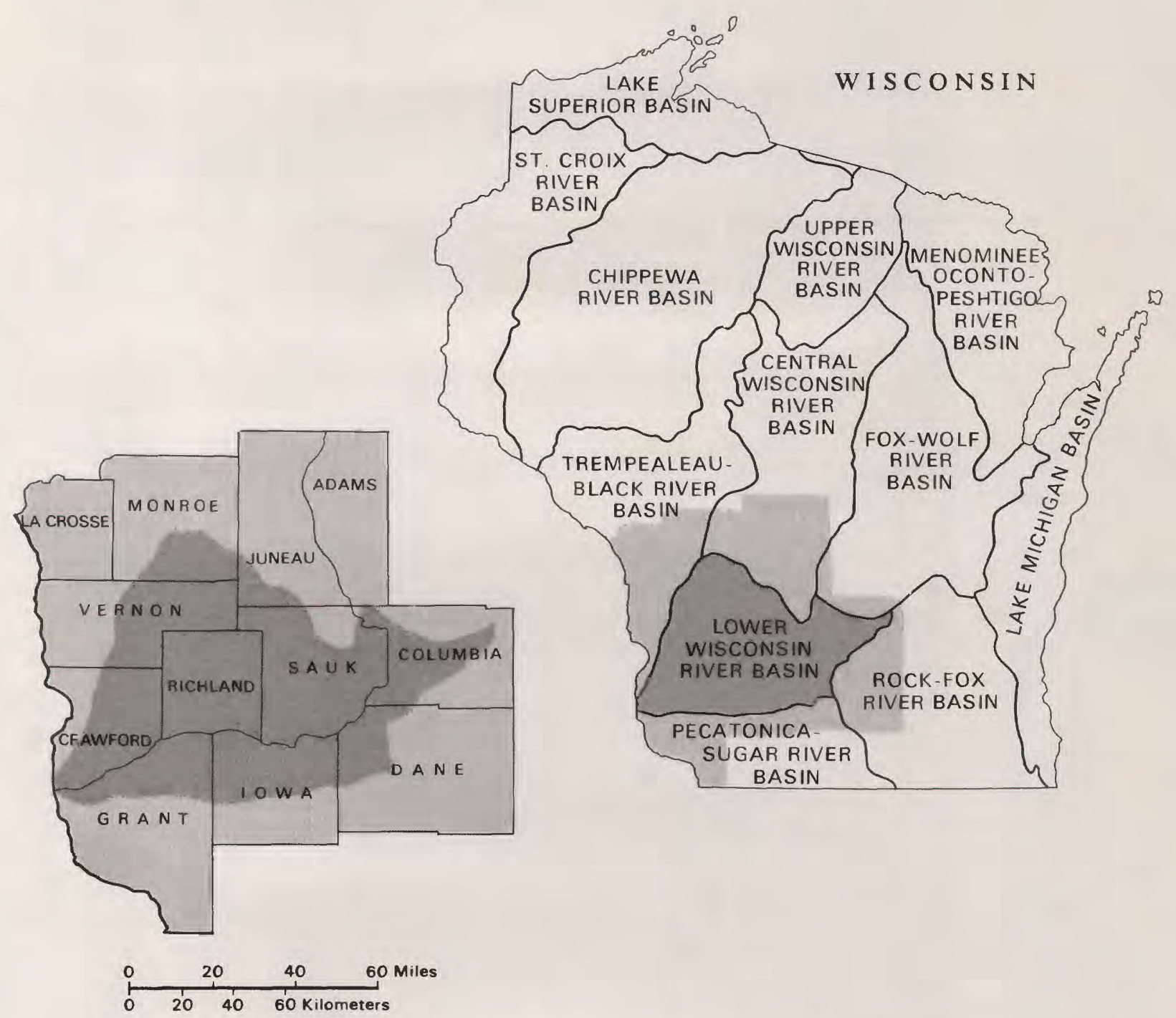

Figure 1. Location of the lower Wisconsin River basin in Wisconsin. 
The report includes: estimates of the magnitude and frequency of recurrence of low flow for various sites where systematic streamflow information has been collected, low-flow discharge measurements that have been obtained at numerous sites throughout the basin, and a method to estimate low-flow characteristics at ungaged sites.

In recent years, a great demand has been placed on water resources in Wisconsin by increased multiple uses such as: maintenance of fish and wildlife habitat, irrigation of crops, dilution and assimilation of wastes, production of hydropower, construction of impoundments for real-estate developments, and maintenance of adequate flow for canoeing. This increased demand requires an accurate determination of water resources during lowflow periods to ensure proper consideration of all users.

Low-flow frequency analyses and flow-duration analyses are presented for all current and discontinued gaging stations in the lower Wisconsin River basin. These analyses have been completed for 11 gaging stations through water year 1975. Low-flow frequency data are included in the report for 26 low-flow partial-record stations and for 70 miscellaneous sites where sufficient data are available.

Previous reports by Gebert and Holmstrom (1974) and Gebert (1971) contain preliminary information on low-flow characteristics of this basin.

For the convenience of readers who may want to use metric units, the data may be converted by using the following factors:

Multiply

miles $(\mathrm{mi})$

feet $(f t)$

square miles $\left(\mathrm{mi}^{2}\right)$

cubic feet per second $(\mathrm{ft} / \mathrm{s})$

feet per mile ( $\mathrm{ft} / \mathrm{mi}$ )

inches (in)

cubic feet per second per

square mile $\left\{(\mathrm{ft} 3 / \mathrm{s}) / \mathrm{mi}^{2}\right\}$

\section{By}

1.609

.3048

2.59

.02832

.1894

2.54

.01094
To obtain

kilometers $(\mathrm{km})$

meters (m)

square kilometers $\left(\mathrm{km}^{2}\right)$

cubic meters per second

$$
(\mathrm{m} 3 / \mathrm{s})
$$

meters per kilometer $(\mathrm{m} / \mathrm{km})$

centimeters $(\mathrm{cm})$

cubic meters per second per square kilometer $\left\{\left(\mathrm{m}^{3} / \mathrm{s}\right) / \mathrm{km}^{2}\right\}$

\section{BASIN DESCRIPTION}

The lower Wisconsin River basin is in southwestern Wisconsin. It includes the entire drainage area of the Wisconsin River from $2 \mathrm{mi}$ south of Wisconsin Dells to its confluence with the Mississippi River. The basin has a drainage area of approximately $3,780 \mathrm{mi}^{2}$, which is about 6.7 percent of the State. 
The basin is mainly a rural agricultural area with a population of about 133,000 people in 1970. The largest cities are Baraboo, Portage, and Richland Center with 1976 populations of $7,931,7,821$, and 5,086, respectively. The economy is primarily agricultural with dairy farming and beef production being the major sources. Crops also are important with vegetables, tobacco, and fruits being primary. Industrial development is generally light in the basin with the major industry being production of dairy products.

The mean annual precipitation for the basin is 32.1 in (U.S. Dept. Commerce, 1931-69), with about 67 percent of this occurring from April through July. Snowfall is about 14 percent of the mean annual precipitation. Hindall and Borman (1974) also found that the mean annual runoff from the basin is about 8.9 in and that the mean annual evapotranspiration is 23.2 in.

The topography differs from the eastern one-fourth of the basin to the western three-fourths owing to the difference in geologic formations. The eastern part of the basin consists of rolling hills and wide valley floors that are formed by unconsolidated glacial and alluvial deposits that overlie the bedrock. In contrast, the western part has rugged, steep-walled. valleys and high relief caused by streams cutting through the sedimentary bedrock. This area is called the "Driftless Area" of Wisconsin because it was not covered by the most recent glaciers. Therefore, it has no drift except reworked sand and gravel deposits in the Wisconsin River valley and thin lake deposits in most of the tributary valleys. Types and locations of glacial deposits are described by Hindall and Borman (1974).

\section{LOW-FLOW CHARACTERISTICS}

Low flow generally refers to the low range of stream discharge. A probability of occurrence and a time period can be specified for a more precise definition. Low flow is usually ground-water runoff or base flow, although a 30-, 60-, or 90-day low flow could contain some direct or storm runoff.

A typical low-flow period is illustrated by the discharge hydrograph for Black Earth Creek at.Black Earth gaging station (fig. 2). The annual 90-day low flow occurs from June 1 to August 29. Although this was the lowest flow for 90 consecutive days during the year, periods of substantial direct runoff occurred on at least three occasions during this period. Except for these rises in stream discharge, the remainder of streamflow for the period was predominantly base flow or ground-water munoff.

Base flow is very important for many low-flow studies because during this period stream discharge is the most stable. Thus, low-flow characteristics can be transferred from a stream where systematic streamflow records have been collected for a period of years to a nearby stream where only a minimum amount of base-flow discharge measurements are available.

Table 1 contains low-flow characteristics for 262 sites in the lower Wisconsin River basin. Each site is identified by station number and 


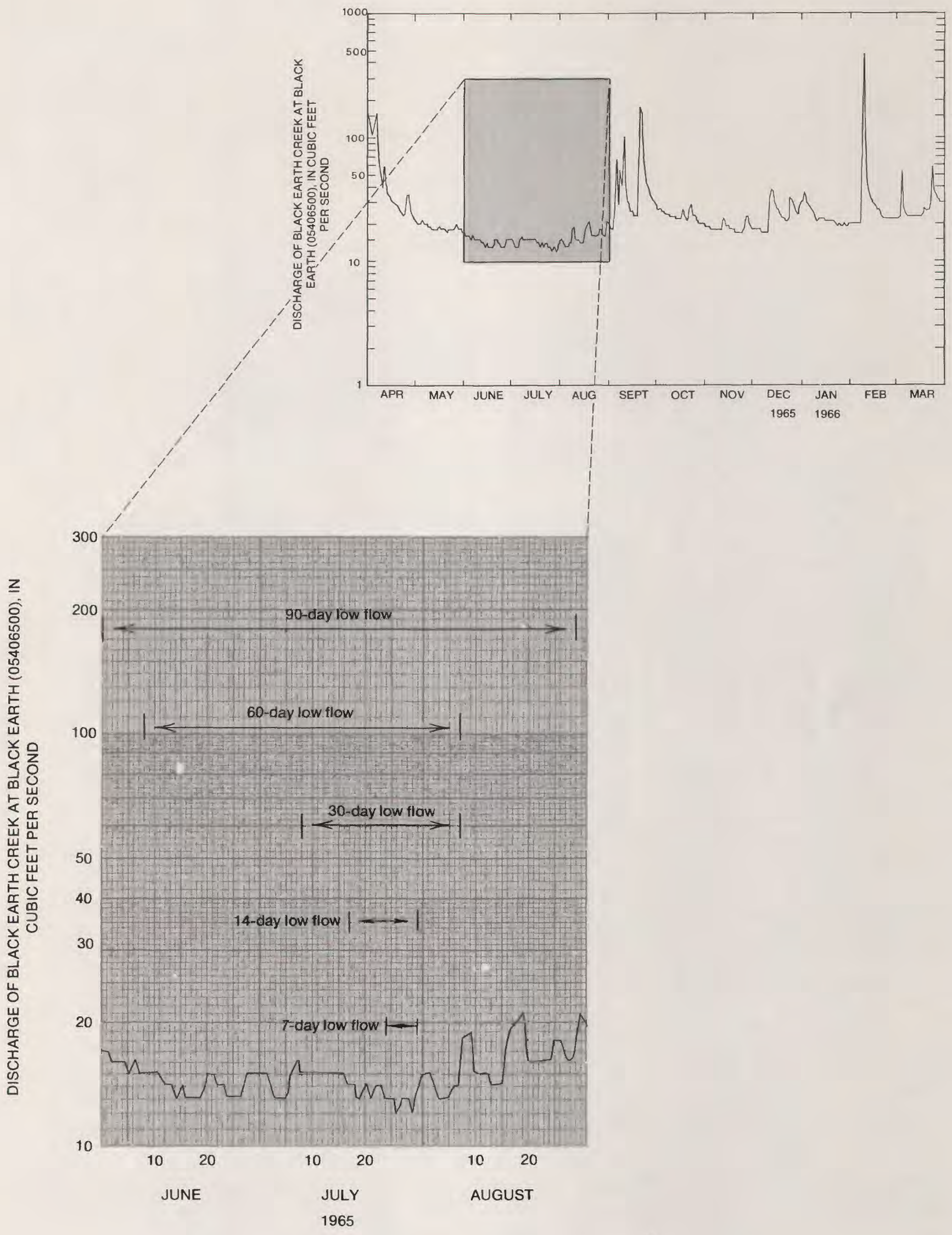

Figure 2. Hydrograph of daily discharge of Black Earth Creek at Black Earth for 1966 climatic year showing annual low-flow periods for various number of days. 
station name. The site location, drainage area, type of site, and other pertinent data are included. Characteristics included for each site depend upon the type of site: gaging station, low-flow partial-record station, or miscellaneous measurement sites. The location of the sites are shown on plate 1.

\section{ANALYTICAL TECHNIQUES}

Low-flow characteristics in table 1 were determined by three methods of analysis. These methods depended on the three basic types of data available: ( 1 ) continuous record of daily streamtlows (continuous-record gaging stations); (2) 8 to 12 base-flow discharge measurements (low-flow partial-record stations); (3) 3 or 4 base-flow discharge measurements (miscellaneous sites).

\section{GAGING STATIONS}

Low-flow characteristics of a stream where systematic streamflow records have been collected can be determined by flow-duration analysis or frequency analysis. The two types of analyses serve different purposes. The flow-duration curve indicates the percent of time that a daily mean flow exceeds a given discharge while the low-flow frequency curve indicates the probability that a 7-day, 14-day, 30-day, 60-day, and 90-day consecutive mean flow will be exceeded in any given year. The recommended and more generally used analysis for most low-flow applications is the low-flow frequency analysis. In the lower Wisconsin River basin the 27,2 discharge is approximately equal to 92 percent flow duration and the 87,10 discharge is about equal to 99 percent flow duration.

Low-flow frequency and flow-duration analyses were completed for all continuous-record gaging stations that have sufficient data: 10 years of record for low-flow frequency analysis and 5 years of record for flowduration analysis. Low-flow frequency values are listed in table 1 showing the magnitude and frequency of annual low flows for $7,14,30,60$, and 90 consecutive days. Table 1 also contains a list of flow-duration values showing the percentage of time that specified discharges were exceeded.

The low-flow frequency characteristics were determined trom the daily discharge records using a log Pearson Type III probability distribution. The results of these analyses were compared to graphs of the annual minimum flows for the various consecutive days (Riggs, 1972). If the two curves did not agree, a graphical interpretation was made to determine the various low-flow characteristics. Figure 3 is an example of a low-flow frequency curve for the Black Earth Creek near Black Earth gaging station, and figure 4 is a flow-duration curve for the same site.

The entire period of recorded discharge was used for the analyses without adjustment for upstream regulation or change in regulation during the period of record. A statement explaining the type of upstream regulation is included in table 1 for sites where substantial regulation exists. 


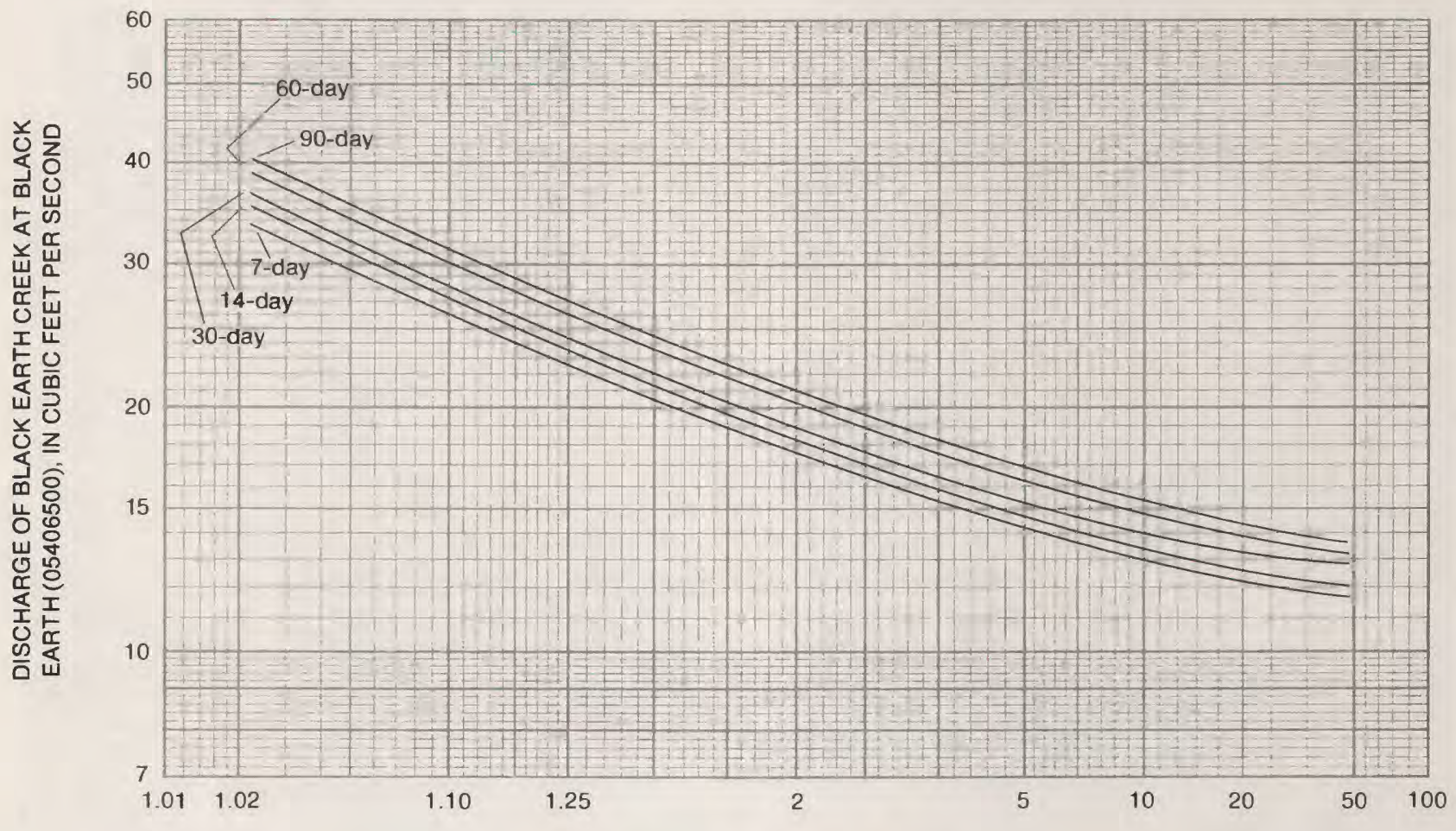

RECURRENCE INTERVAL, IN YEARS

Figure 3. Low-flow frequency curves showing the magnitude and frequency of the annual mi nimum mean discharge for the indicated number of consecutive days at Black Earth Creek.

For gaging stations that have insufficient data for low-flow frequency analysis or flow duration, the low-flow characteristics were determined by a procedure similar to that outlined in the following section for low-flow partial-record stations.

\section{LOW-FLOW PARTIAL-RECORD STATIONS}

Low-flow characteristics determined for low-flow partial-record stations are the annual minimum 7-day mean flow below which the flow will fall on the average of once in 2 years $(27,2)$ and once in 10 years $(27,10)$. Estimates of 27,2 and 87,10 are presented in table 1 for 26 low-flow partialrecord stations. Characteristics were determined fror a relation line established by correlating 8 to 12 base-flow discharge measurements at lowflow partial-record stations with concurrent discharges at continuousrecord gaging stations in the area (Gebert, 1971). The Q7,2 and 87,10 at the continuous-record gaging station then were transferred through the relation line to estimate 27,2 and 87,10 for the partial-record station. Figure 5 is an example of this type of analysis for Otter Creek near Clyde. 


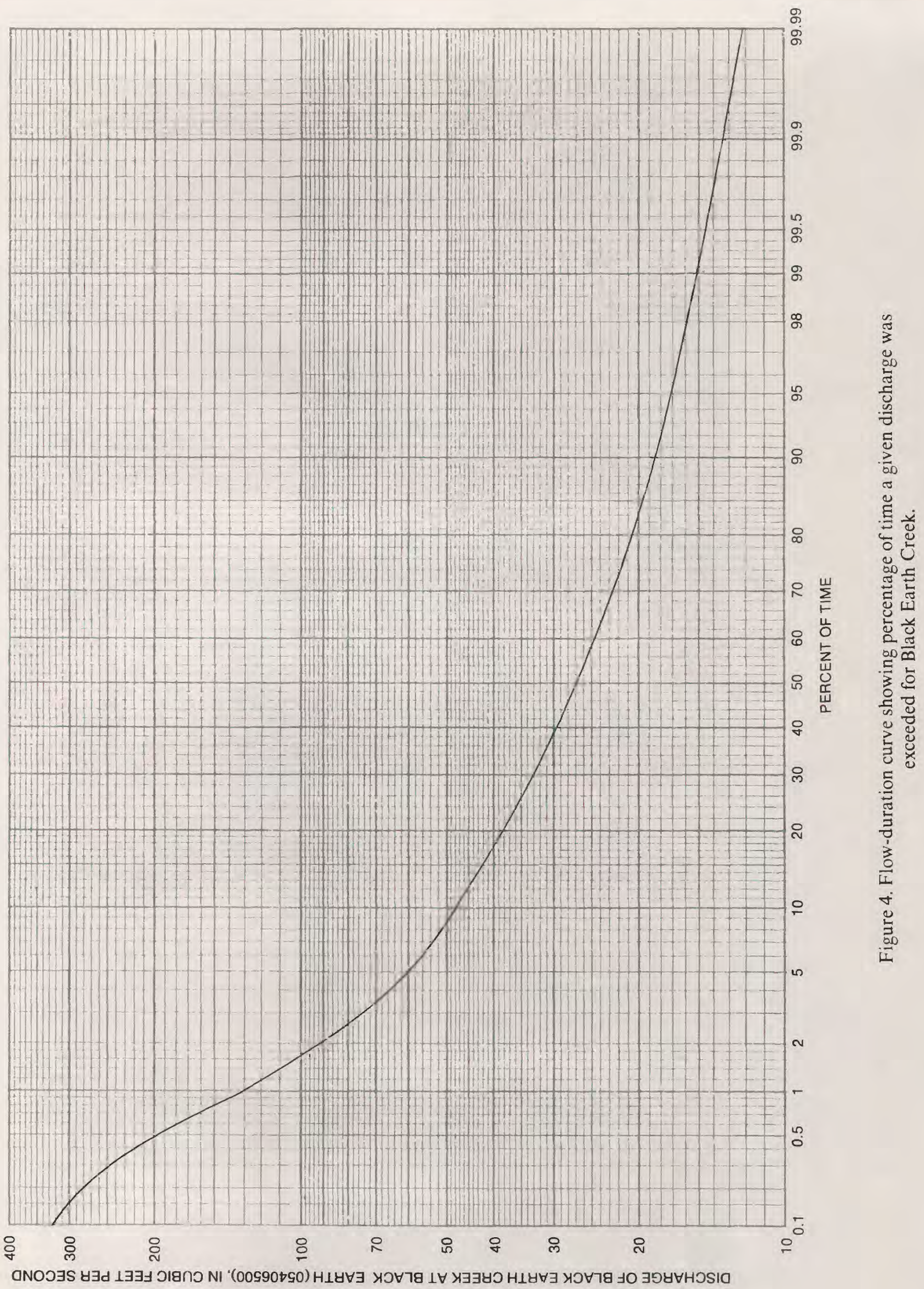




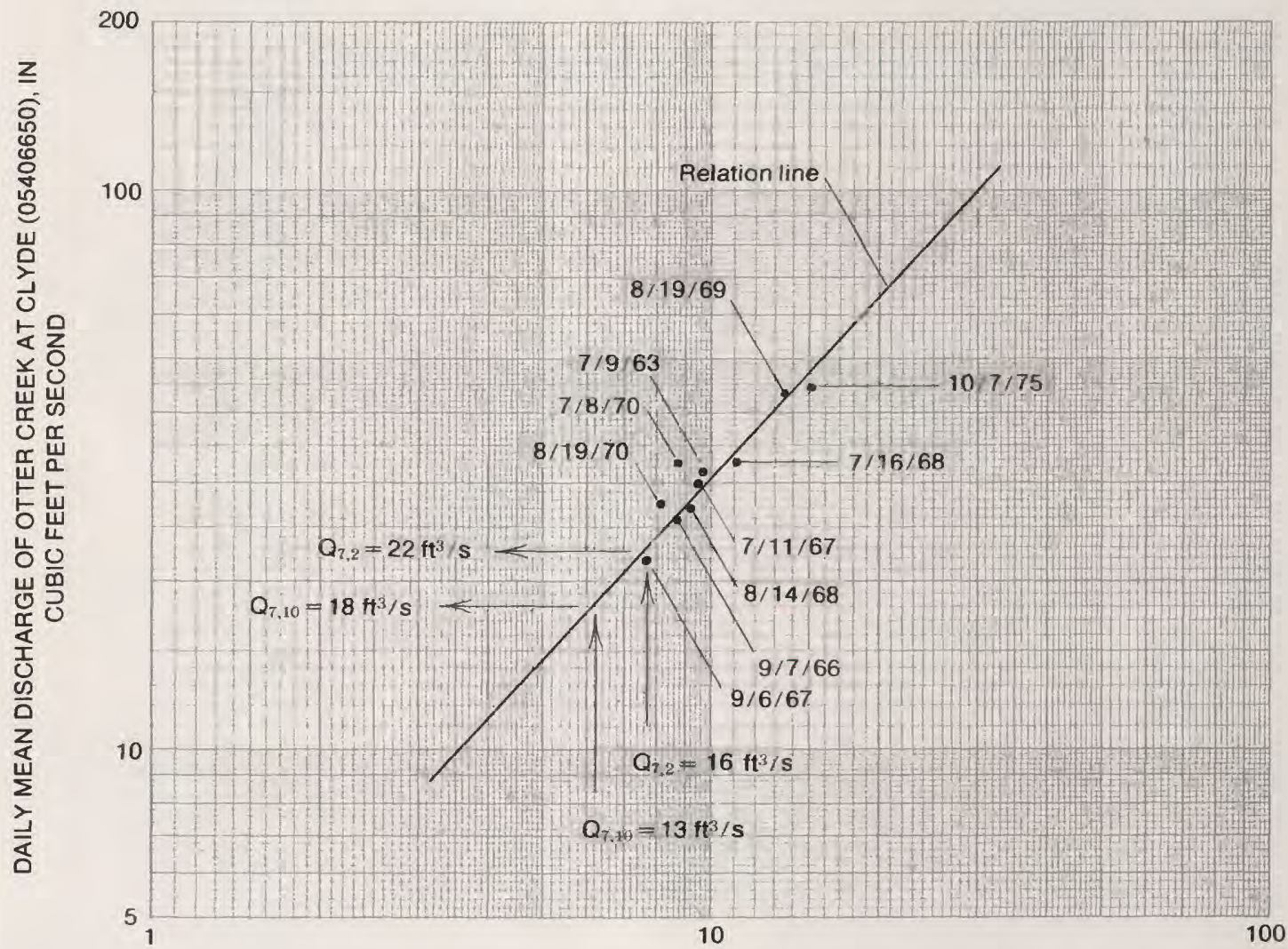

DISCHARGE OF BLACK EARTH CREEK AT BLACK EARTH (05406500), IN CUBIC FEET PER SECOND

Figure 5. Method of estimating $\mathrm{Q}_{7,2}$ and $\mathrm{Q}_{7,10}$ at low-flow partial-record stations from correlation of base-flow discharge measurements at Otter Creek with concurrent daily mean discharge of Black Earth Creek. 


\section{MISCELLANEOUS SITES}

Base-flow measurements have been obtained at 225 miscellaneous sites in the lower Wisconsin River basin as part of other water-resource investigations. Low-flow characteristics were estimated for these sites (table l) if at least three base-flow discharge measurements were available and a well-defined relationship existed between the measured discharge and the concurrent daily mean discharge at a nearby gaging station. Estimates of Q7,2 and Q7,10 were made by the same type of analysis that was used for partial-record stations (Gebert and Holmstrom, 1974). Figure 6 illustrates this type of analysis for West Branch Honey Creek near Plain. The slope of the relation line for miscellaneous sites was compared to established relation lines of nearby low-flow partial-record stations and other miscellaneous sites for uniformity. Generally the relation line should have approximately the same slope if the factors that influence low flow are uniform for the area. If the relation line at the site being studied was defined by three discharge measurements that had significant scatter, the line was adjusted to agree more closely with the better established relation line at a low-flow partial-record station.

At some sites, the low-flow characteristics were not determined because the relationship between measurements at the miscellaneous site and concurrent daily mean discharge at a gaging station was poorly defined. An inadequate relationship generally was due to poor base-flow measurement conditions or an insufficient number of discharge measurements.

For those sites where low-flow characteristics were not determined, the measured base-flow discharges are listed in table 1 to provide a range of observed base flows.

\section{ACCURACY}

The low-flow characteristics in table 1 are estimates of flow expected in the future. Low-flow characteristics like other streamflow characteristics are only estimates, with their true value being difficult or impossible to determine. The estimates are based on data collected at each site and analyzed by several methods. Each estimate has an error associated with it, dependent on the amount and kind of data, and the analytical method. Two major sources of error are the time-sampling error in streamflow records and the error in the analytical method.

The expected degree of accuracy for the 47,2 and 27,10 estimates are presented in table 1 for each site. The accuracy is determined by the standard error of estimate for the 7-day, 2-year low flow $\left(\mathrm{SE}_{7}, 2\right)$ and for the 7-day, 10-year low flow $\left(\mathrm{SE}_{7}, 10\right)$. The standard error of estimate is a range such that the values estimated by the method are within this range at about 67 percent of the sites, and are within twice this range at about 95 percent of the sites. An explanation of how the accuracy was determined for each method follows. 


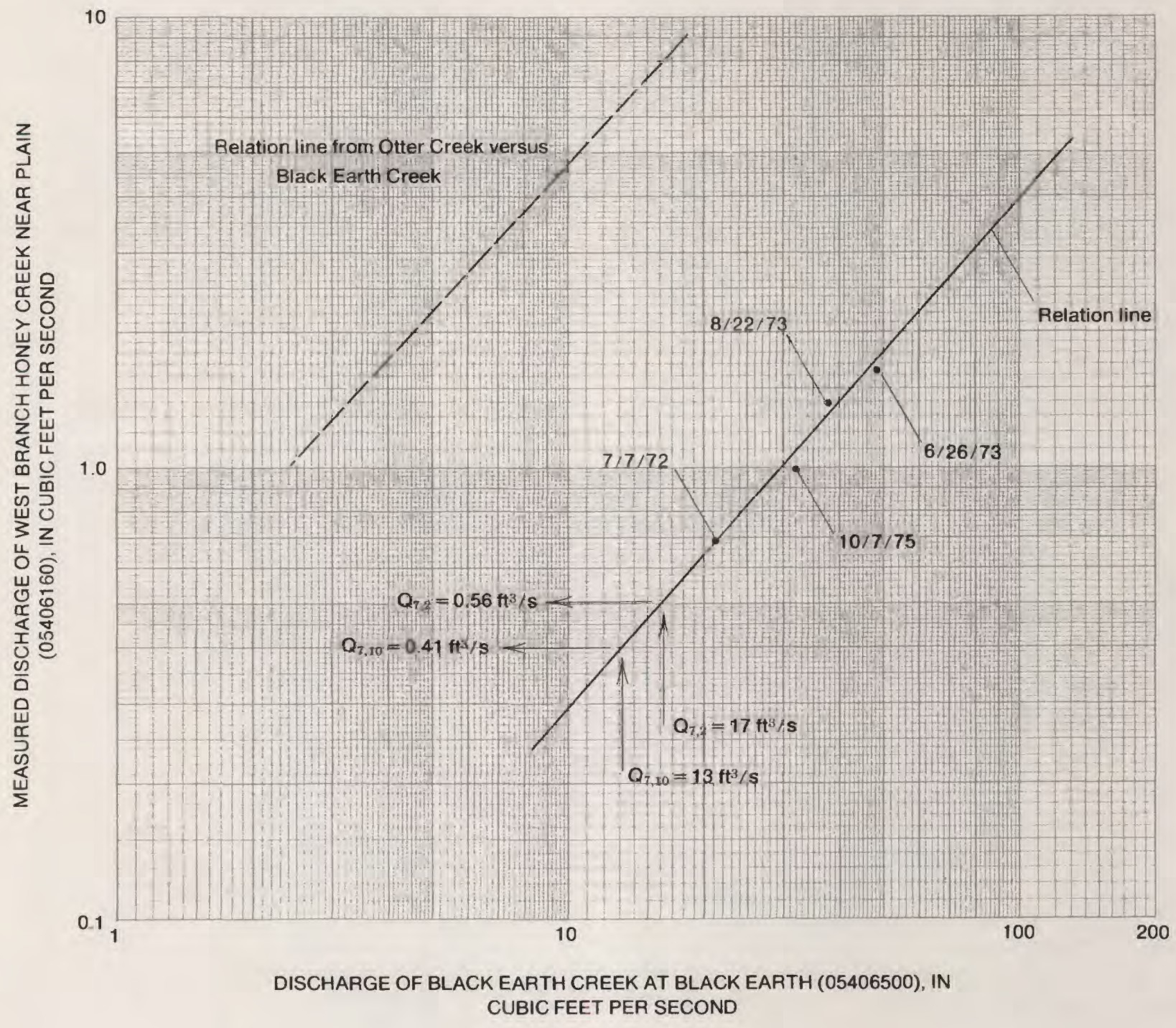

Figure 6. Method of estimating $\mathrm{Q}_{7,2}$ and $\mathrm{Q}_{7,10}$ at miscellaneous sites from correlation of baseflow discharge measurements at West Branch Honey Creek with concurrent daily mean discharge of Black Earth Creek. 


\section{GAGING STATIONS}

Accuracy of low-flow characteristics at gaging stations was determined according to Hardison (1969). An average $\mathrm{SE}_{7,2}$ of 5 percent and $\mathrm{SE}_{7}, 10$ of 7 percent was determined for the six gaging stations in the lower wisconsin River basin that had greater than 10 years of unregulated-streamflow record.

A common length of record was used to compare the accuracy of low-flow characteristics determined from recorded discharge at gaging stations in the lower Wisconsin River basin with that of gaging stations throughout the State. This analysis assumed that 10 years of record was available at each gaging station to determine the $\mathrm{Q}_{7,10}$ discharge. An $\mathrm{SE}_{7,10}$ of 11 percent was determined for the lower Wisconsin River basin as compared to an $\mathrm{SE}_{7,10}$ of 16 percent for gaging stations throughout the State. This significant difference indicates that low-flow characteristics generally can be determined much more accurately in the lower Wisconsin River basin.

\section{LOW-FLOW PARTIAL-RECORD STATIONS}

The accuracy of low-flow characteristics at low-flow partial-record stations was determined by a method developed by Hardison and Moss (1972). Using this method, an average $\mathrm{SE}_{7,10}$ of 11 percent was found for the 26 low-flow partial-record stations in the lower Wisconsin River basin. This compares to an average $\mathrm{SE}_{7,10}$ of 29 percent for 265 low-flow partialrecord stations throughout the State.

Hardison and Moss also developed a method for evaluating the accuracy at low-flow partial-record stations in terms of equivalent years of continuous streamflow record. The method izcludes the number of discharge measurements available at a low-flow partial-record station, length of record at the gaged site, and accuracy of the regression that relates the discharge measurements to the recorded discharges. A greater number of discharge measurements generally will give a more accurate estimate of the low-flow characteristics. The goal for the low-flow study is to provide estimates of low-flow characteristics at low-flow partial-record stations which are equivalent to 10 years of continuous streamflow record.

The average equivalent years of record for the 26 low-flow partialrecord stations in the lower Wisconsin River basin was determined to be about 10 years, which meets the desired accuracy goal. This determination was based on an average of 8 to 12 base-flow measurements at each low-flow partial-record station. Additional measurements would be required at 9 of the 26 low-flow partial-record stations for the accuracy goal to be met at every site.

\section{MISCELLANEOUS SITES}

The accuracy of low-flow characteristics at miscellaneous sites was determined as an average value for the entire basin by analyzing data collected at low-flow partial-record stations. 
Three random base-flow measurements were selected from the 8 to 12 measurements available at the 26 low-flow partial-record stations. Lowflow characteristics were determined from these three measurements using the same procedure used for miscellaneous sites. Then low-flow characteristics determined by this method were plotted against the low-flow characteristics based on 8 to 12 measurements. The SE between the two methods was determined from this plotted relationship. The overall SE includes the SE determined by the plotted relationship and the SE associated with the low-flow estimates based on 8 to 12 measurements. Assuming the two errors are independent, the overall SE can be approximated by taking the square root of the sum cf the squares of the two different SE's. For the lower Wisconsin River basin this resulted in an $\mathrm{SE}_{7,10}$ of 19 percent which is listed in table 1 as tro average basin accuracy. The average $\mathrm{SE}_{7,10}$ value should be used cautiously for any particular site since the actual value for a subbasin could be significantly different from the mean for the basin. If the low-flow characteristics are based on more than three discharge measurements, the accuracy probably will be improved and should approach the accuracy at Icrflow partial-record stations as additional measurements are obtained.

\section{ESTIMATING LOW-FLOW CHARACTERISTICS AT UNGAGED SITES}

A method is required to transfer low-flow characteristics from gage sites to ungaged sites because it is impossible to obtain actual streamflow data for all sites where the information is needed. The most practical transfer method relates low-flow characteristics to topographic and climatic characteristics of the drainage basin by multiple-regression analysis. Characteristics used in the multiple-regression analysis and the equatiors determined are discussed in the following paragraphs. The method is outlined in detail by Thomas and Benson (1970).

\section{STREAMFLOW CHARACTERISTICS}

Streamflow characteristics that were studied are the $Q_{7,2}$ and $0_{7,10}$ which are widely used to describe low flow. The multiple-regression analysis included low-flow characteristics for 2 gaging stations and 26 low-flow partial-record stations in the lower Wisconsin River basin. The streamflow characteristics are the dependent variable in the multipleregression analysis.

\section{DRAINAGE-BASIN CHARACTERISTICS}

The variation of streamflow for different locations and with time is caused by the differences in precipitation patterns and the differences in runoff characteristics. Climatic and topographic characteristics are drainage-basin characteristics and are quantified to explain the variation in low flow. These indices or drainage-basin characteristics are the independent variables in the multiple-regression analysis.

Drainage-basin characteristics were selected for the analyses because of their known influence on the rainfall-runoff process. The following 
list of the drainage-basin characteristics contains a brief discussion of their effect on low flow and how the indices were developed.

Values for these drainage-basin characteristics for low-flow partialrecord stations and selected gaging stations in the lower Wisconsin River basin are listed in table 2 .

Drainage area (A).--Size of the drainage area is the most significant characteristic in explaining differing streamflow between sites. Because low flow is ground-water runoff, the contributing area is defined by the ground-water divide of a basin which can be determined from potentiometric maps. Because potentiometric maps are not available for most areas in the lower Wisconsin River basin, the surface-water divide was used to define the contributing drainage area. Due to the relatively steep relief in the basin, the difference between the area of ground-water drainage and surfacewater drainage is probably slight.

Drainage areas, in square miles, were computed from U.S. Geological Survey topographic maps or similar topographic maps of comparable size and detail. Drainage-area data for this study were obtained from Holmstrom (1972).

Main-channel slope (S).--Main-channel slope (Benson, 1962 and 1964) is a characteristic that relates to the change in streamflow for different basins. The index of slope used in this analysis is the average slope in feet per mile between points 10 percent and 85 percent of the distance upstream from the gaged site to the drainage-basin divide.

Main-channel length (L).--Main-channel length is another landform characteristic that indicates basin shape in conjunction with drainage area of the basin. In estimating ground-water runoff to the stream, $I$ can be viewed as describing the length of the vertical cross-sectional area of the porous aquifer material through which the flow occurs. Channel length was obtained from the U.S. Geological Survey topographic maps by measuring the total indicated blue-line length by a digitizer, divider, or other means.

Basin storage (BS).--Basin storage is that part of total drainage area occupied by lakes and marshes. Variations in streamflow can be caused by retention and release of water from basin storage. For some streams, runoff is delayed by storage, but total runoff may not be reduced; whereas on other streams prolonged retention allows increased evapotranspiration that results in decreased runoff. Essentially, the basin storage index is used in the analysis to reflect the effect of evapotranspiration on low flow.

The basin storage area was obtained from U.S. Geological Survey topographic maps. A value of 1.00 percent was added to all values of basin storage to avoid problems of using zero in the regression analysis. 
Forest cover (F).--Forests affect streamflow in several ways. Their major influences on low flow are intercepting precipitation before it reaches the ground and using water through transpiration. In the "sand plain" area of Wisconsin, Weeks and Stangland (1971) found that converting 10 percent of the headwater drainage area from grassland to forest would reduce the late summer streamflow by about 5 percent.

The forest cover index used in this analysis is the percentage of drainage area covered by forests as shown on U.S. Geological Survey topographic maps. A value of 1.00 percent was added to all values of forest cover to avoid problems of using zero in the regression analysis.

Mean annual precipitation (P).--Mean annual precipitation of a basin expresses the amount of water available for potential runoff. The precipitation that infiltrates the soil and passes through the unsaturated zone to the ground-water supply is the source of base flow for a stream. The mean annual precipitation, in inches, for each basin was computed from an isohyetal map determined from precipitation recorded at U.S. Weather Bureau stations.

A constant of 20 in was subtracted from each value for use in the regression analysis. This reduction provides constants and exponents in the regression equation that are more manageable.

Soil-infiltration rate (I).--Soil permeability influences the amount of direct runoff from a storm and the amount of water that infiltrates the soil. The permeability used is an average rate for the basin under average soil and moisture conditions.

Soil types and average permeability, in inches per hour, for each basin were determined from maps by Hindall and Borman (1974).

Mean annual snowfall (Sn).--Mean annual snowfall, like mean annual precipitation, is an indicator of water available for runoff. For each basin an average mean annual snowfall, in inches, was determined from a map of Wisconsin weather (Wisconsin Statistical Reporting Service, 1967). A constant of 20 in was subtracted from each value to provide more manageable constants and exponents in the equations.

Base-flow index (Bf).--A good indicator of a stream's low-flow potential is a discharge measurement made during base-flow conditions. Base-flow measurements provide considerable information about the characteristics of the aquifers supplying outflow to the stream. Because a large number of base-flow measurements have been made in the basin, it was desirable to use these data.

To use base-flow measurements, it is necessary to convert them to a uniform base because measurements generally are obtained at various points on the base-flow recession curves. Therefore, the measurements were used to determine a base-flow index value to represent a common base flow. 
Discharge at the 90 percent flow duration was selected to represent the base-flow index value. To evaluate the technique and develop the necessary relationships for this study, sites were selected that had discharge measurements obtained for a low-flow investigation during the period August $17-19,1970$.

Measured discharges $\left(Q_{m}\right)$ were converted to a unit discharge by dividing the values by their respective drainage area (A). These values then were adjusted by a basin ratio to determine the base-flow index for each site. Basin ratios were determined for gaging stations on unregulated streams within the lower Wisconsin River basin by dividing the discharge at 90 percent flow duration (Q90) by the observed average daily discharge during August, 1719, $1970\left(Q_{r}\right)$. Thus, base-flow index values were determined by the equation:

$$
B f=\frac{Q_{m} Q_{90}}{A Q_{r}}
$$

Plate 2 shows the locations of 91 sites with base-flow index values, their respective drainage-area outlines, and their computed base-flow index values. Values were obtained for 26 low-flow partial-record stations and 2 gaging stations used in the multiple-regression analysis. In most cases, discharges had been obtained at the low-flow partial-record stations during August 17-19, 1970, therefore, only a minimum amount of interpolation was needed to compute the Bf values.

\section{REGRESSION ANALYSIS}

Multiple-regression analysis was used to determine the relationship between the streamflow characteristics (dependent variables) and the basin characteristics (independent variables). The analysis provides an equat: 0 , or series of equations, relating the dependent to the independent variables. This analysis defined mathematical equations of the form:

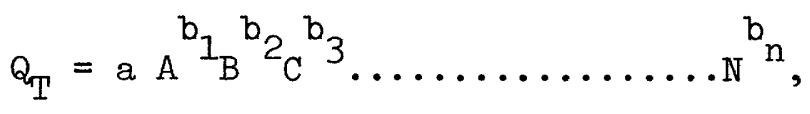

where: $Q_{T}$ is a 7 -day low-flow characteristic having a T-yeaj
recurrence interval, in cubic feet per second;

a is a regression constant defined by the regression analysis;

$A B C \ldots . N$ are drainage-basin characteristics; and

$b_{1} b_{2} b_{3} \cdots b_{n}$ are regression coefficients defined by regression

The analysis also defined the standard error of estimate (SE) of the analytical method and the statistical significance of each variable in the equation. 
The standard error of estimate is a measure of the accuracy of the regression relationships. It describes a range in error between the defined relationship and the data included in the analysis. Values estirated by the regression equations are within the range of one standard error of estimate at about 67 percent of the sites and within twice this range for 95 percent of the sites.

The statistical significance of each variable is an indication of the probability that the effect of the variable is real. Those variables the.t were significant at the 95 percent confidence level were considered significant to the equation and those at the 99 percent confidence level were considered to be highly significant.

Step-forward regression analyses were performed by digital computer using procedures outlined by Thomas and Benson (1970). The equations with the lowest standard error of estimate with all variables significant at the 99 percent or the 95 percent confidence level were selected as the best equations for prediction.

Drainage area, forest cover, soil-infiltration rate, and base-flow index were found the most significant characteristics in explaining the variations in low flow. Although other characteristics may logically explain or quantify the precipitation-runoff process, they do not vary across the area studied and therefore cannot explain the difference that occurs in low flow. Mean annual precipitation is an example of this type of characteristic that does not vary enough across the basin. Also absent from the final results are two of the principal characteristics that influence low flow: geology and evapotranspiration. It was not possible to describe these characteristics adequately for the analysis.

Two sets of analyses were performed to develop equations for sites with no streamflow data available and for sites with minimum streamflow data available. One analysis included all the drainage-basin characteristics except for base-flow index, and the other analysis contained all the drainage-basin characteristics including the base-flow index.

\section{SITES WITHOUT STREAMFLOW DATA}

The two equations selected from the analyses for these sites are:

$$
\begin{aligned}
& Q_{7,2}=0.218 \mathrm{~A}^{1.05} \\
& Q_{7,10}=0.165 \mathrm{~A}^{1.04} \\
& Q_{7,2} \text { is the 7-day, 2-year low flow in cubic feet per second; } \\
& Q_{7,10} \text { is the 7-day, 10-year low flow in cubic feet per second; and } \\
& \text { A is drainage area in square miles. }
\end{aligned}
$$


The standard error of estimate is 44 percent for equation (1) and 52 percent for equation (2). These SE reflect the accuracy or ability of the equation to fit the observed data. To compare the accuracy of these equations with the accuracy listed for gaging stations, low-flow partialrecord stations, and miscellaneous sites listed in table 1 , the values were adjusted to account for the errors associated with the 27,2 and $Q_{7,10}$ values used to develop the equations. The average $\mathrm{SE}_{7,2}$ for the $\mathrm{Q}_{7,2}$ values is 9 percent and the average $\mathrm{SE}_{7,10}$ for the $\mathrm{Q}_{7,10}$ values is 11 percent for the gaging stations and low-flow partial-record stations used in the analysis. The overall SE's for the equations are approximated by taking the square root of the sum of the squares of the two different SE's. This results in an $\mathrm{SE}_{7,2}$ of 45 percent for equation (1) and an $\mathrm{SE}_{7,10}$ of 54 percent for equation (2).

\section{SITES WITH MINIMUM STREAMFLOW DATA}

Two sets of equations were selected from the analysis for use. The equations and their respective standard error of estimate are:

\section{Equation}

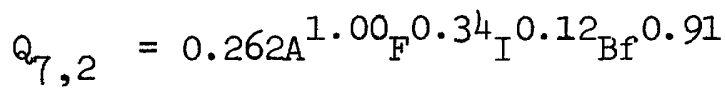

$$
\begin{aligned}
& Q_{7,2}=0.571 \mathrm{~A}^{1.07} \mathrm{Bf}^{0.87} \\
& Q_{7,10}=0.172 \mathrm{~A}^{0.98} \mathrm{~F}^{0.45} \mathrm{I}^{0.19} \mathrm{Bf}^{1.02} \\
& Q_{7,10}=0.463 \mathrm{~A}^{1.07} \mathrm{Bf}^{0.95}
\end{aligned}
$$

Equations (3) and (5) are the recommended equations because they have lower standard error of estimates. Equations (4) and (6) are provided because they have only a slightly higher standard error and are easier to apply.

$$
\begin{aligned}
& \text { Q7,2, } Q_{7,10} \text {, and } A \text { are as defined for equations }(1) \text { and }(2) ; \\
& F \text { is the percentage of drainage area covered by forest plus } \\
& 1.00 \text { percent; } \\
& \text { I is the average soil-infiltration rate for a basin in inches per } \\
& \text { hour; and }
\end{aligned}
$$

$\mathrm{Bf}$ is the base-flow index in cubic feet per second per square mile.

Equation (5) was tested using streamflow data collected August 20, 1969, at low-flow partial-record stations during a high base-flow period and September 9, 1967, during a low base-flow period. Values of Bf were obtained as outlined previously. Substituting these new values of Bf into equation (5), estimates of $Q_{7,10}$ were determined for low-flow partialrecord stations. When compared to the $Q 7,10$ values listed in Table 1 , the 
$\mathrm{SE}_{10}$ for the high base-flow period was 30 percent and the $\mathrm{SE}_{10}$ for the low base-flow period was 37 percent. Both of these estimates included the 11 percent average SE associated with the 47,10 values listed in Table 1 . Thus, equation (5) produces satisfactory results for other sets of flow conditions than were used for its development.

Equations (3), (4), (5), and (6) should provide estimates of 47,2 and Q7,10 within the SE indicated for sites where the Bf has already been determined (pl. 2) and for other sites where base-flow discharge measurements could be used to develop Bf values. These equations also may be expectel to provide more reliable estimates than equations (1) and (2) for stream: that are in areas where a high degree of uniformity exists between $B f$ values as shown on plate 2 .

\section{APPLICATION OF ESTIMATING PROCEDURES}

\section{SITES WITHOUT STREAMFLOW DATA}

Computation of low-flow characteristics at an ungaged site may be made as follows:

1. Use equations (1) and (2) listed on page 17 to determine the 1owflow characteristics.

2. Compute the drainage area as indicated on page 14 .

3. Substitute these drainage-area values into equations (1) and (2) and solve for the low-flow characteristics.

For example, to determine the low-flow characteristics of Big Rock Branch near Highland at the mouth:

The applicable equations for an ungaged area are:

$$
\begin{aligned}
Q_{7,2} & =0.218 \mathrm{~A}^{1.05} \\
Q_{7,10} & =0.165 \mathrm{~A}^{1.04}
\end{aligned}
$$

Determine the drainage area for this site as outlined on page 14 of this report, substituting this value into the respective equation:

$$
\begin{aligned}
Q_{7,2} & =0.218 \mathrm{~A}^{1.05} \\
& =(0.218)(9.96)^{1.05} \\
& =(0.218)(11.2) \\
& =2.44 \mathrm{ft}^{3} / \mathrm{s}
\end{aligned}
$$




$$
\begin{aligned}
Q_{7,10} & =0.165 \mathrm{~A}^{1.04} \\
& =(0.165)(9.96)^{1.04} \\
& =(0.165)(10.9) \\
& =1.80 \mathrm{ft}^{3} / \mathrm{s}
\end{aligned}
$$

\section{SITES WITH MINIMUM STREAMFLOW DATA AVAILABLE}

Computation of the low-flow characteristics at sites with minimum streamflow data available is made as follows:

1. Use equations (3) and (5) listed on page 18 to determine the lowflow characteristics.

2. Determine from plate 1 and table 1 the type of streamflow data that are available.

3. If streamflow measurements were made during the August 17-19, 1970, period, the base-flow index (Bf) can be selected from plate 2 .

4. If the streamflow measurements were made for some other period during base-flow conditions, the Bf should be determined as outlined on page 15.

5. Compute the other basin characteristics, drainage area, soil index, and forest cover, used in the equation as outlined on pages 14 and 15.

6. Substitute values determined in steps 3 or 4 along with step 5 into equations (3) and (5).

As an example to determine the low-flow characteristics of Camp Creek near Viola (station number 05408300), the following procedure would be used:

The applicable equations for an ungaged area with minimum streamflow data available are:

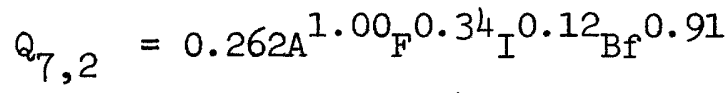

$$
\begin{aligned}
& Q_{7,10}=0.172 A^{0.98} F^{0.45} I^{0.19} B^{1.02}
\end{aligned}
$$

Drainage area (A) obtained from table 1 , page 70 , is $15.7 \mathrm{mi}^{2}$. 
The measured forested area is $5.44 \mathrm{mi}^{2}$. Therefore, $F=\frac{5.44}{15.7} \times 100+$ 1 percent $=35.6$ percent.

The soil-infiltration rate for the basin according to Hindall and Borman (1974) has a range from $0.05-0.2 \mathrm{in} / \mathrm{h}$ under a 0.5 in head. Using an average value, I is $0.12 \mathrm{in} / \mathrm{h}$.

The base-flow index obtained from plate 2 is 0.54 .

Substituting these values into their respective equations:

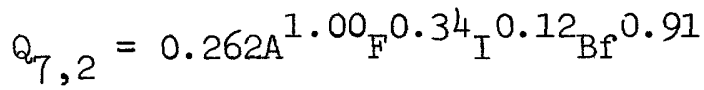

$$
\begin{aligned}
& =(0.262)(15.7)^{1.00}(35.6)^{0.34}(0.12)^{0.12}(0.54)^{0.91} \\
& =(0.262)(15.7)(3.37)(0.775)(0.571) \\
& =6.13 \mathrm{ft}^{3} / \mathrm{s} \\
& Q_{7,10}=0.172 \mathrm{~A}^{0.98} \mathrm{~F}^{0.45} \mathrm{I}^{0.19} \mathrm{Bf}^{1.02} \\
& =(0.172)(15.7)^{0.98}(35.6)^{0.45}(0.12)^{0.19}(0.54)^{1.02} \\
& =(0.172)(14.9)(4.99)(0.67)(0.53) \\
& =4.54 \mathrm{ft}^{3} / \mathrm{s}
\end{aligned}
$$

To determine low-flow characteristics at sites where streamflow measurements are available but not for the August 17-19, 1970, period, the following procedure at the site, Pine River near Yuba (station number $05406704)$, is used as an example.

The equations to use are the same as the above example:

$$
\begin{aligned}
Q_{7,2} & =0.262 A^{1.00} F^{0.34} I^{0.12} B_{f^{0.91}} \\
Q_{7,10} & =0.172 A^{0.98} F^{0.45} I^{0.19} B_{f^{1.02}}
\end{aligned}
$$

Drainage area (A) obtained from table 1 , page 46 , is $9.68 \mathrm{mi}^{2}$.

The measured forested area is $4.26 \mathrm{mi}^{2}$. Therefore, $F=\frac{4.26}{9.68} \times 100+$ 1 percent $=45$ percent.

An average soil-infiltration rate for the basin was determined from the soil permeability map from Hindall and Borman (1974) to be 1.3 in/h. 
The base-flow index cannot be obtained from plate 2 because a baseflow measurement was not obtained during the August 17-19, 1970, period. Therefore, a Bf value has to be determined from the base-flow measuremerts that are available. Two measurements were made at this site (table 1 ). Following the same general procedure indicated on pages 15 and 16 , a $\mathrm{Bf}$ value was determined by the equation:

$$
B f=\frac{Q_{m} Q_{90}}{A Q_{r}}
$$
where: $\quad Q_{m}$ is the measured discharge, $4.36 \mathrm{ft}^{3} / \mathrm{s}$, of Pine River near
Yuba on October 17, 1972;

$\mathrm{A}$ is the drainage area, $9.68 \mathrm{mi}^{2}$, of Pine River near Yuba;

$Q_{r}$ is the recorded discharge at a nearby continuous-record gaging station. Referring to plate 1, station 05408000, Kickapco River at LaFarge is the closest gaging station. From Water Resources Data for Wisconsin (1973) the average daily discharge for October 17, 1972, was $163 \mathrm{ft} 3 / \mathrm{s}$; and

$\mathrm{Q}_{90}$ for Kickapoo River at LaFarge is $81 \mathrm{ft}^{3} / \mathrm{s}$, obtained from
table 1 .

Substituting these values in the equation:

$$
\begin{aligned}
\mathrm{Bf} & =\frac{Q_{m} Q_{90}}{A Q_{r}} \\
& =\frac{(4.36)(81)}{(9.68)(163)} \\
& =0.224
\end{aligned}
$$

The low-flow characteristics then can be determined by substituting: these values in their respective equations.

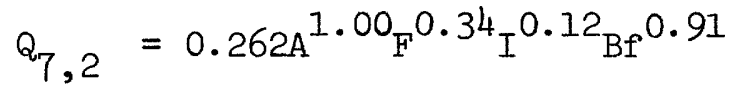

$$
\begin{aligned}
& =(0.262)(9.68)^{1.00}(45)^{0.34}(1.3)^{0.12}(0.224)^{0.91} \\
& =(0.262)(9.68)(3.65)(1.03)(0.256) \\
& =2.44 \mathrm{ft}^{3} / \mathrm{s}
\end{aligned}
$$




$$
\begin{aligned}
Q_{7,10} & =0.172 \mathrm{~A}^{0.98} \mathrm{~F}^{0.45} \mathrm{I}^{0.19} \mathrm{Bf}^{1.02} \\
& =(0.172)(9.68)^{0.98}(45)^{0.45}(1.3)^{0.19}(0.224)^{1.02} \\
& =(0.172)(9.25)(5.55)(1.05)(0.217) \\
& =2.01 \mathrm{ft}^{3} / \mathrm{s}
\end{aligned}
$$

\section{COMPARISON OF METHODS}

If estimates of low-flow characteristics are required at sites other than those presented in this report, the user interested in the data should evaluate the need for the low-flow information. Generally the most important criteria in choosing a method are: accuracy requirements of the low-flow characteristics; time available to collect and analyze data; and cost of data collection and analyses.

Table 3 compares the methods available and provides: type of data required; number of sites where required data are available; time required to collect data; analytical method used to determine the low-flow characteristics; and standard error of estimate associated with the method. If a high degree of reliability is required of low-flow characteristics and sufficient time is available for data collection, a gaging station or lowflow partial-record station should be operated. If a lesser degree of reliability is acceptable at a site or time and money are a factor, thre $\epsilon$. base-flow discharge measurements should be obtained or one of the regression equations may be sufficient.

\section{CONCLUSIONS}

For the 26 low-flow partial-record stations and 2 gaging stations with drainage areas less than $150 \mathrm{mi}^{2}$, the average $0_{7,2}$ is 0.28 ( $\left.\mathrm{ft}^{3} / \mathrm{s}\right) / \mathrm{mi} 2$ ard the average $Q 7,10$ is $0.22(\mathrm{ft} 3 / \mathrm{s}) / \mathrm{mi}^{2}$. These basin averages are significantly higher than the State averages of $0.20(\mathrm{ft} 3 / \mathrm{s}) / \mathrm{mi}^{2}$ for 27,2 and $0.14(\mathrm{ft} 3 / \mathrm{s}) / \mathrm{mi}^{2}$ for the $\mathrm{Q}_{7,10}$.

The low-flow characteristics can be determined adequately by the five methods included'in this report, especially when compared to other basins in the state. This is attributed to the relative uniformity of the factcrs that control low flow, mainly precipitation, geology, and evapotranspiration.

Using the methods described, the low-flow characteristics determinec at partial-record stations achieved the desired goal of having an average. accuracy equivalent to 10 years of record. This accuracy was based on or $1 \mathrm{y}$ 8 to 12 discharge measurements at each site. 
Multiple-regression equations also were developed, and they made $i$, possible to determine the low-flow characteristics at ungaged sites with an acceptable degree of accuracy. Two sets of equations were determined, one for use at sites without any additional streamflow data and the other for sites with base-flow measurements. The latter equation had an $\mathrm{SE}_{7}, 10$ of 32 percent compared to 54 percent for the former. The most significant characteristics in explaining the variation in low flow were found to be drainage area, forest cover, soil-infiltration rate, and base-flow inder.

\section{REFERENCES}

Benson, M. A., 1962, Factors influencing the occurrence of floods in a humid region of diverse terrain: U.S. Geol. Survey Water-Supply Paper $1580-B, 64 \mathrm{p}$.

1964, Factors affecting the occurrence of floods in the Southwest: U.S. Geol. Survey Water-Supply Paper 1580-D, 72 p.

Gebert, W. A., 1971, Low-flow frequency of Wisconsin streams: U.S. Geol. Survey Hydrol. Inv. Atlas HA-390.

Gebert, W. A., and Holmstrom, B. K., 1974, Low-flow characteristics of Wisconsin streams at sewage-treatment plants: U.S. Geol. Survey Water-Resources Inv. 45-74, $101 \mathrm{p}$.

Hardison, C. H., 1969, Accuracy of streamflow characteristics in Geological Survey research 1969: U.S. Geol. Survey Prof. Paper 650-D, p. D210214.

Hardison, C. H., and Moss, M. E., 1972, Accuracy of low-flow characteristics estimated by correlation of base-flow measurements: U.S. Geol. Survey Water-Supply Paper 1542-B, 55 p.

Hindall, S. M., and Borman, R. G., 1974, Water resources of Wisconsin-lower Wisconsin River basin: U.S. Geol. Survey Hydrol. Inv. Atlas HA479.

Holmstrom, B. K., 1972, Drainage-area data for Wisconsin streams: U.S. Geol. Survey open-file report.

Riggs, H. C., 1972, Low-flow investigations: U.S. Geol. Survey Techniques Water-Resources Inv., book 4, chap. BI, $18 \mathrm{p}$.

Thomas, D. M., and Benson, M. A., 1970, Generalization of streamflow characteristics from drainage-basin characteristics: U.S. Geol. Survey Water-Supply Paper 1975, $55 \mathrm{p}$.

U.S. Department of Commerce, Weather Bureau, 1931-69, Climatological data: Washington, D. C., U.S. Govt. Printing Office. 
U.S. Geological Survey, 1974, Water resources data for Wisconsin, 1973: U.S. Geol. Survey Ann. Rept., 262 p.

Weeks, E. P., and Stangland, H. G., 1971, Effects of irrigation on streamflow in the central sand plain of Wisconsin: U.S. Geol. Survey open-file report, $113 \mathrm{p}$.

Wisconsin Statistical Reporting Service, 1967, Wisconsin weather: Madison, Wisconsin Statistical Reporting Service, 31 p. 
Table 1.--Low-flow characteristics for sites in the lower Wisconsin River basin

05404025 Middle Branch Duck Creek near Rio, Wis.

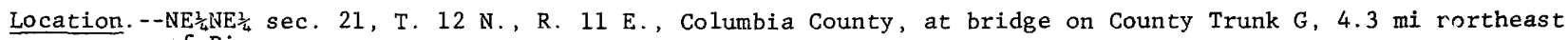
of Rio.

Drainage area. $-7.72 \mathrm{mi}^{2}$.

Tributary to. - -Wisconsin River.

Type of site.--Miscellaneous site.

Discharge measurement.--Aug. 18, 1970, $0.71 \mathrm{ft}^{3} / \mathrm{s}$.

05404027 Middle Branch Duck Creek near Rio, wis.

Location. - -SW $\frac{1}{4} \mathrm{SW}_{\frac{1}{4}} \mathrm{sec} .19$, T. $12 \mathrm{~N} .$, R. $11 \mathrm{E}$. , Columbia County, at bridge on town road, $2.9 \mathrm{mi}$ north of Rio. Drainage area. $-28.0 \mathrm{mi}^{2}$. Tributary to.--Duck Creek.

Type of site. - Miscellaneous site.

Discharge measurement. --Aug. $18,1970,7.12 \mathrm{ft}^{3} / \mathrm{s}$.

054040277 North Branch Duck Creek at Cambria, Wis.

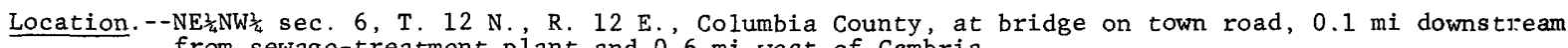
from sewage-treatment plant and $0.6 \mathrm{mi}$ west of Cambria.

Drainage area. $--8.55 \mathrm{mi}^{2}$. Tributary to. --Wisconsin River.

Type of site. --Miscellaneous site.

Discharge measurements.--July 5, 1972, $0 \mathrm{ft} / \mathrm{s} ;$ Oct. $101975,0.96 \mathrm{ft}^{3} / \mathrm{s}$.

Low-flow frequency. $-\mathrm{Q}_{7,2}=0 \mathrm{ft}^{3} / \mathrm{s}, \mathrm{Q}_{7,10}=0 \mathrm{ft}^{3} / \mathrm{s}$.

05404029 North Branch Duck Creek near Cambria, Wis.

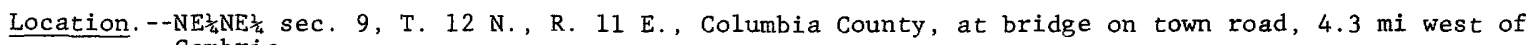
Cambria.

Drainage area. $--28.0 \mathrm{mi}^{2}$.

Tributary to.--Duck Creek.

Type of site.--Miscellaneous site.

Discharge measurement. --Aug. 18, 1970, $2.24 \mathrm{ft}^{3} / \mathrm{s}$.

05404030 North Branch Duck Creek near Pardeeville, Wis.

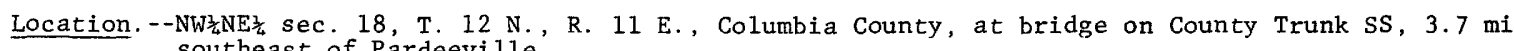
southeast of Pardeeville.

Drainage area. $--33.6 \mathrm{mi}^{2}$. Tributary to. --Duck Creek.

Type of site.--Low-flow partial-record station.

Minimum discharge measured. $--3.16 \mathrm{ft}^{3} / \mathrm{s}$, Aug. 13, 1964.

Low-flow frequency. $-Q_{7,2}=5.4 \mathrm{ft}^{3} / \mathrm{s}, Q_{7,10}=3.3 \mathrm{ft} / \mathrm{s}$.

Basis of estimate.--Correlated with Baraboo River near Baraboo using 14 discharge measurements made in period 1962-67.

Accuracy. $-\mathrm{SE}_{7,10}=13$ percent. 
05404033 Duck Creek at Wyocena, Wis.

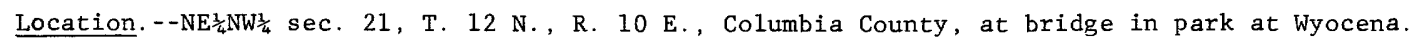

Drainage area. $--79.6 \mathrm{mi}^{2}$.

Tributary to. --Wisconsin River.

Type of site.--Miscellaneous site.

Discharge measurements. - -July 5, 1972, 20.7 $\mathrm{ft}^{3} / \mathrm{s}$; June 25, 1973, $43.3 \mathrm{ft}^{3} / \mathrm{s}$; Aug. $21,1973,35.3 \mathrm{ft}^{3} / \mathrm{s}$; Oct. $10,1975,17.9 \mathrm{ft}^{3} / \mathrm{s}$.

Low-flow frequency. - $-Q_{7,2}-15 \mathrm{ft}^{3} / \mathrm{s}, Q_{7,10}=12 \mathrm{ft}^{3} / \mathrm{s}$.

Basis of estimate. --Correlated with Baraboo River near Baraboo using 4 discharge measurements.

Accuracy. $--\mathrm{SE}_{7,10}=19$ percent (average value for basin).

05404039 Baraboo River at Kenda11, Wis .

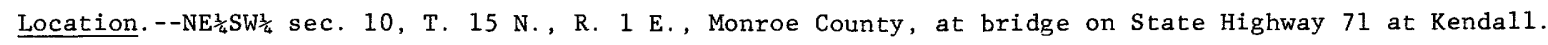

Drainage area. $--15.9 \mathrm{mi}^{2}$. Tributary to. - -Wisconsin River.

Type of site.--Miscellaneous site.

Discharge measurements.--June 13, 1973, $10.7 \mathrm{ft}^{3} / \mathrm{s} ;$ July $25,1973,7.96 \mathrm{ft}^{3} / \mathrm{s} ; 0 \mathrm{ct} .24,1973,6.38 \mathrm{ft}^{3} / \mathrm{s}$.

Low-flow frequency. $-\mathrm{Q}_{7,2}=2.4 \mathrm{ft}^{3} / \mathrm{s}, \mathrm{Q}_{7,10}=1.7 \mathrm{ft}^{3} / \mathrm{s}$.

Basis of estimate.--Correlated with Baraboo River near Baraboo using 3 discharge measurements.

Accuracy. $--\mathrm{SE}_{7,10}=19$ percent (average value for basin).

05404040 Baraboo River at Kendal1, Wis.

Location. -- $\begin{aligned} & \frac{1}{4} \mathrm{NE}_{\frac{1}{4}} \mathrm{sec} .15, \text { T. } 15 \mathrm{~N} ., \text { R. } 1 \text { E., Monroe County, at bridge on State Highway } 71,0.9 \mathrm{mi} \text { southeast } \\ & \text { of Kendall. }\end{aligned}$

Drainage area. $--16.4 \mathrm{mi}^{2}$. Tributary to. - -Wisconsin River.

Type of site.--Miscellaneous site.

Discharge measurement.--Aug. 18, 1970, $1.75 \mathrm{ft}^{3} / \mathrm{s}$.

05404050 Cleaver Creek tributary near Elroy, Wis.

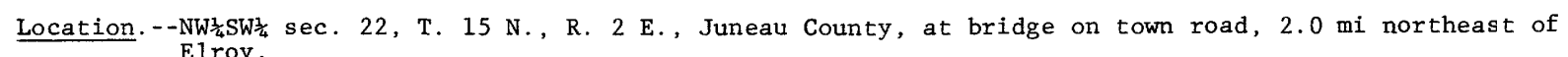
Drainage area. $--4.16 \mathrm{mi}^{2}$.

Tributary to --Cleaver Creek.

Type of site.--Miscellaneous site.

Discharge measurement.--Aug. 17, 1970, $0.28 \mathrm{ft}^{3} / \mathrm{s}$.

05404080 Baraboo River at Elroy, Wis.

Location. - -NW $\frac{1}{4} \mathrm{SW}_{\frac{1}{4}} \mathrm{sec} .33$, T. 15 N., R. 2 E., Juneau County, at bridge on County Trunk 0, at E1roy.

Drainage area. $--59.4 \mathrm{mi}^{2}$.

Tributary to. - -Wisconsin River.

Type of site. --Miscellaneous site

Discharge measurements. - -June $14,1973,37.9 \mathrm{ft}^{3} / \mathrm{s} ;$ Oct. $24,1973,27.0 \mathrm{ft}^{3} / \mathrm{s} ;$ June $22,1976,19.4 \mathrm{ft}^{3} / \mathrm{s}$; Aug. 10, $1976,16.3 \mathrm{ft}^{3} / \mathrm{s}$.

Low-flow frequency. $-Q_{7,2}=12 \mathrm{ft}^{3} / \mathrm{s}, Q_{7,10}=9.1 \mathrm{ft}^{3} / \mathrm{s}$.

Basis of estimate.--Correlated with Baraboo River at Baraboo using 5 discharge measurements made during the period 1973-76.

Accuracy. $--\mathrm{SE}_{7,10}=19$ percent (average value for the basin). 
05404085 Baraboo River at E1roy, Wis.

Location. - - SW $\frac{3}{4} \mathrm{SW}_{\frac{1}{4}}$ sec. 33, T. 15 N., R. 2 E., Juneau County, at bridge on State Highways 80 and 82 , at Elroy.

Drainage area. $--59.5 \mathrm{mi}^{2}$.

Tributary to. --Wisconsin River.

Type of site.--Miscellaneous site.

Discharge measurement.--July $25,1973,33.8 \mathrm{ft}^{3} / \mathrm{s}$.

05404100 Baraboo River near Union Center, Wis.

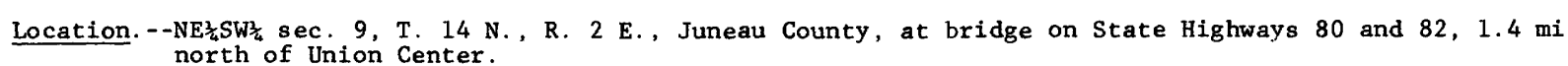
Drainage area. $--73.1 \mathrm{mi}^{2}$. Tributary to. - -Wisconsin River.

Type of site.--Low-flow partial-record station.

Minimum discharge measured. $--10.6 \mathrm{ft}^{3} / \mathrm{s}$, Sept. $7,1967$.

Low-flow frequency. $-Q_{7,2}=13 \mathrm{ft}^{3} / \mathrm{s}, Q_{7,10}=9.6 \mathrm{ft}^{3} / \mathrm{s}$.

Basis of estimate.--Correlated with Baraboo River near Baraboo using 9 discharge measurements made in Accuracy. $--\mathrm{SE}_{7,10}=11$ percent.

05404105 Seymour Creek at Union Center, Wis.

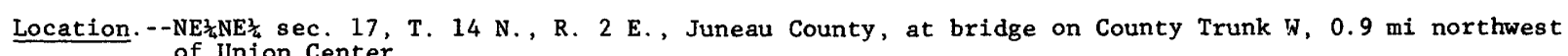
of Union Center.

Drainage area. $--21.5 \mathrm{mi}^{2}$. Tributary to. --Baraboo River.

Type of site.--Miscellaneous site.

Discharge measurement.--Aug. $17,1970,4.13 \mathrm{ft}^{3} / \mathrm{s}$.

05404110 Baraboo River at Union Center, Wis.

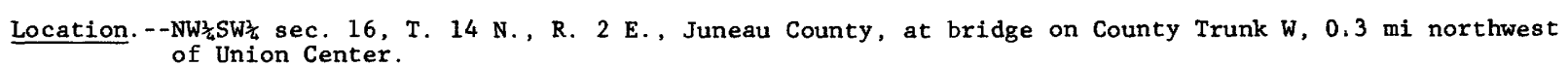
Drainage area. $--97.2 \mathrm{mi}^{2}$. Tributary to. - -Wisconsin River.

Type of site.--Miscellaneous site.

Discharge measurement. --Aug. $17,1970,16.7 \mathrm{ft}^{3} / \mathrm{s}$.

05404112 Baraboo River at Union Center, Wis.

Location. - -SE $\frac{3}{4} \mathrm{NW}_{\frac{3}{4}}$ sec. $21, \mathrm{~T} .14 \mathrm{~N} ., \mathrm{R} .2 \mathrm{E}$, Juneau County, at village of Union Center sewage-treatnent plant.

Drainage area. $--97.8 \mathrm{mi}^{2}$. $\quad$ Tributary to.--Wisconsin River.

Type of site.-Miscellaneous site.

Discharge measurements.--June $23,1976,27.6 \mathrm{ft}^{3} / \mathrm{s}$; Aug. $10,1976,29.6 \mathrm{ft}^{3} / \mathrm{s}$.

05404116 South Branch Baraboo River at Hillsboro, Wis.

Location. - $-\mathrm{NE}_{4} \mathrm{NE} \frac{3}{4} \mathrm{sec} .35, \mathrm{~T} .14 \mathrm{~N}$, , R. 1 E., Vernon County, at bridge on County Trunk FF, at Hillsboro.

Drainage area. $--39.1 \mathrm{mi}^{2}$. Tributary to.--Baraboo River.

Type of site.--Miscellaneous site.

Minimum discharge measured. $--9.40 \mathrm{ft}^{3} / \mathrm{s}$, Aug. 10,1976 .

Low-flow frequency. $-\mathrm{Q}_{7,2}=5.2 \mathrm{ft}^{3} / \mathrm{s}, \mathrm{Q}_{7,10}=3.1 \mathrm{ft}^{3} / \mathrm{s}$.

Basis of estimate.--Correlated with Baraboo River near Baraboo using 6 discharge measurements.

Accuracy. $--\mathrm{SE}_{7,10}=19$ percent (average value for basin). 
05404118 Hills Creek near Hillsboro, Wis.

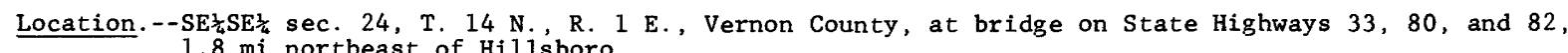

Drainage area. $--16.2 \mathrm{mi}^{2}$. Tributary to.--South Branch Baraboo River.

Type of site.--Miscellaneous site.

Discharge measurement.--Dec. 8, $1970,4.91 \mathrm{ft}^{3} / \mathrm{s}$.

05404120 South Branch Baraboo River tributary near Hillsboro, Wis.

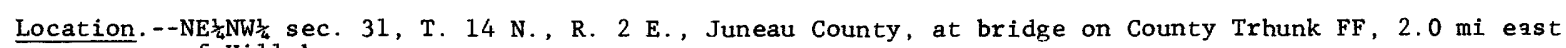
of Hills boro.

Drainage area. $--1.62 \mathrm{mi}^{2}$.

Tributary to. --South Branch Baraboo River.

Type of site.--Miscellaneous site.

Discharge measurement.--Aug. $17,1970,0.18 \mathrm{ft}^{3} / \mathrm{s}$.

05404123 Baraboo River at Wonewoc, Wis.

Location.--SW $\frac{1}{4} N W \frac{1}{4}$ sec. 35, T. 14 N., R. 2 E., Juneau County, at bridge on town road at Wonewoc.

Drainage area. $--174 \mathrm{mi}^{2}$.

Tributary to.--Wisconsin River.

Type of site.--Miscellaneous site.

Discharge measurements.--July $25,1973,96.8 \mathrm{ft}^{3} / \mathrm{s} ;$ June $23,1976,49.0 \mathrm{ft} / \mathrm{s} ; \mathrm{Aug} .10,1976,46.5 \mathrm{ft} / \mathrm{s}$. Low-flow frequency. $--Q_{7,2}=32 \mathrm{ft}^{3} / \mathrm{s}, Q_{7,10}=21 \mathrm{ft}^{3} / \mathrm{s}$.

Basis of estimate. --Correlated with Baraboo River near Baraboo using 3 discharge measurements.

Accuracy. $--\mathrm{SE}_{7,10}=19$ percent (average value for basin).

05404125 Gardner Creek near Wonewoc, Wis.

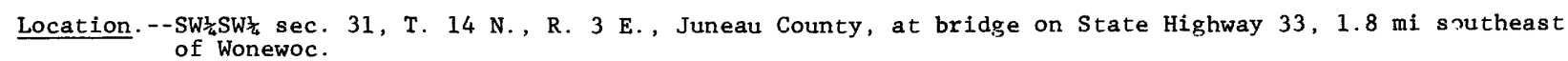
Drainage area. $--4.45 \mathrm{mi}^{2}$. Tributary to. --Baraboo River.

Type of site.--Miscellaneous site.

Discharge measurement.--Aug. $17,1970,0.16 \mathrm{ft}^{3} / \mathrm{s}$.

05404127 Crossman Creek near LaValle, Wis.

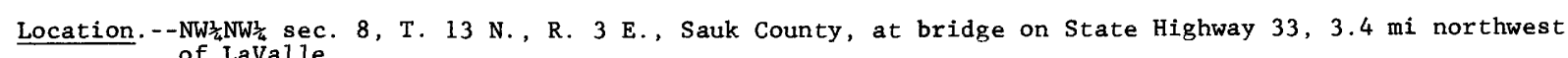
of LaValle.

Drainage area. $--19.9 \mathrm{mi}^{2}$.

Tributary to. --Baraboo River.

Type of site.--Miscellaneous site.

Discharge measurement.--Dec. $8,1970,2.35 \mathrm{ft}^{3} / \mathrm{s}$.

05404132 McGlynn Creek at Cazenovia, Wis.

Location. - $-\mathrm{NE}_{\frac{1}{4}} \mathrm{NE}_{\frac{1}{4}}$ sec. 13, T. 12 N., R. 2 E., Richland County, at State Highway 58 bridge at Cazenovia.

Drainage area. $-21.5 \mathrm{mi}^{2}$.

Tributary to.--Little Baraboo River.

Type of site.--Miscellaneous site.

Discharge measurement. --June 22, 1976, $7.62 \mathrm{ft}^{3} / \mathrm{s}$. 
05404133 McGlynn Creek near Cazenovia, Wis.

Location. - $-\mathrm{NW}_{\frac{3}{4}} \mathrm{NW} \frac{3}{4}$ sec. 8 , T. $12 \mathrm{~N}$, R. 3 E., Sauk County, 0.2 mi upstream from bridge on State Highway 58 and $1.6 \mathrm{mi}$ northeast of Cazenovia.

Drainage area. $--23.9 \mathrm{mi}^{2}$. Tributary to.--Little Baraboo River.

Type of site.--Miscellaneous site. Discharge measurements.--June 23, 1976, 7.30 $\mathrm{ft}^{3} / \mathrm{s} ;$ Aug. 9, 1976, 5.90 $\mathrm{ft}^{3} / \mathrm{s} ; \mathrm{Aug} .30,1976,5.16 \mathrm{ft} / \mathrm{s}$,

05404134 McGlynn Creek near Ironton, Wis.

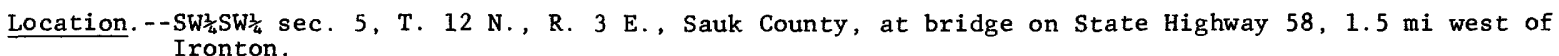

Drainage area. $--33.5 \mathrm{mi}^{2}$.

Tributary to. - - Baraboo River.

Type of site.--Miscellaneous site.

Discharge measurement. --Aug. $17,1970,2.26 \mathrm{ft}^{3} / \mathrm{s}$.

05404137 Little Baraboo River near LaValle, Wis.

Location. $-\mathrm{SE}_{\frac{1}{4} \mathrm{NE} \frac{1}{4}}$ sec. 33, T. $13 \mathrm{~N}$, R. 3 E., Sauk County, at bridge on State Highway 58 , 1.4 mi south of LaValle.

Drainage area. $--69.0 \mathrm{mi}^{2}$. Tributary to.--Baraboo River.

Type of site.--Miscellaneous site.

Discharge measurement. - -Dec. $8,1970,16.8 \mathrm{ft}^{3} / \mathrm{s}$.

05404140 West Branch Big Creek near LaValle, Wis.

Location. - $-\mathrm{SE}_{\frac{1}{4}} \mathrm{SW}_{\frac{1}{4}} \mathrm{sec} .36$, T. $14 \mathrm{~N}$, R. $3 \mathrm{E}$, Juneau County, at bridge on town road, $4.8 \mathrm{mi}$ northeast of LaValle.

Drainage area. $--11.9 \mathrm{mi}^{2}$.

Tributary to.--Baraboo River.

Type of site.--Miscellaneous site.

Discharge measurement.--Aug. $17,1970,1.18 \mathrm{ft}^{3} / \mathrm{s}$.

05404150 Baraboo River tributary near LaValle, Wis .

Location. - $-\mathrm{SW} \frac{1}{4} \mathrm{SE} \frac{1}{4} \mathrm{sec} .24, \mathrm{~T} .13 \mathrm{~N}$, R. $3 \mathrm{E}$., Sauk County, at bridge on County Trunk V, $2.4 \mathrm{mi}$ east of Drainage area. $--1.5 \mathrm{mi}^{2}$. Tributary to. --Baraboo River.

Type of site.--Miscellaneous site.

Discharge measurements.--Aug. 17, 1970, $0.185 \mathrm{ft}^{3} / \mathrm{s} ;$ Dec. 8, 1970, $0.255 \mathrm{ft}^{3} / \mathrm{s}$.

05404165 Hay Creek near Reedsburg, Wis.

Location. $-\mathrm{SE}_{\frac{1}{4}} \mathrm{SW} \frac{1}{4} \mathrm{sec} .34, \mathrm{~T} .13 \mathrm{~N}$, R. 4 E., Sauk County, at bridge on private road, $1.6 \mathrm{mi}$ north of Reedsburg.

Drainage area. $--8.21 \mathrm{mi}^{2}$.

Tributary to. --Baraboo River.

Type of site.--Miscellaneous site.

Discharge measurement.--Dec. 8, 1970, $1.94 \mathrm{ft}^{3} / \mathrm{s}$. 
05404200 Narrows Creek at Loganville, Wis.

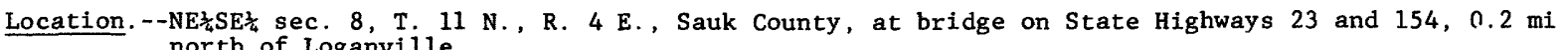
north of Loganville.

Drainage area. $--40.1 \mathrm{mi}^{2}$. Tributary to. - - Baraboo River.

Type of site.--Low-flow partial-record station.

Minimum discharge measured. $--1.66 \mathrm{ft}^{3} / \mathrm{s}, \mathrm{July} 27,1965$.

Low-flow frequency. $-Q_{7,2}=3.6 \mathrm{ft}^{3} / \mathrm{s}, Q_{7,10}=2.7 \mathrm{ft}^{3} / \mathrm{s}$.

Basis of estimate.--Correlated with Baraboo River near Baraboo using 58 discharge measurements made in period 1961-73.

Accuracy. $--\mathrm{SE}_{7,10}=8$ percent.

05404205 Narrows Creek tributary near Loganville, Wis.

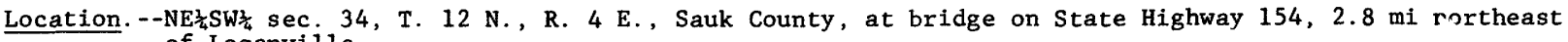
of Loganville.

Drainage area. $--4.57 \mathrm{mi}^{2}$.

Tributary to. --Narrows Creek.

Type of site.--Miscellaneous site.

Discharge measurements. --June 13, 1973, 2.19 $\mathrm{ft}^{3} / \mathrm{s} ;$ July $24,1973,1.54 \mathrm{ft}^{3} / \mathrm{s} ; 0 \mathrm{ct} .25,1973,1.72 \mathrm{ft}^{3} / \mathrm{s}$;

Low-flow frequency. $-Q_{7,2}=0.74 \mathrm{ft}^{3} / \mathrm{s}, Q_{7,10}=0.58 \mathrm{ft}^{3} / \mathrm{s}$.

Basis of estimate.--Correlated with Baraboo River near Baraboo using 5 discharge measurements.

Accuracy. - $-\mathrm{SE}_{7,10}=19$ percent (average value for basin).

05404250 Narrows Creek near Rock Springs, Wis.

Location.--SW $\frac{1}{4} N W \frac{1}{4} \mathrm{sec} .32$, T. 12 N., R. 5 E., Sauk County, at bridge on State Highway $154,0.9 \mathrm{mi}$ rest of Rock Springs.

Drainage area. $--68.1 \mathrm{mi}^{2}$.

Tributary to. --Baraboo River.

Type of site.--Miscellaneous site.

Discharge measurement.--Dec. $8,1970,15.8 \mathrm{ft}^{3} / \mathrm{s}$.

05404400 Seeley Creek near North Freedom, Wis.

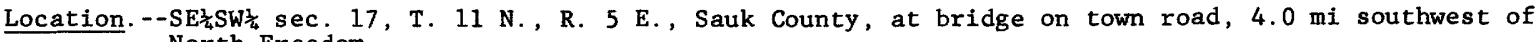
North Freedom.

Drainage area. $--12.4 \mathrm{mi}^{2}$.

Tributary to. --Baraboo River.

Type of site.--Miscellaneous site.

Discharge measurement. --Aug. $18,1970,1.60 \mathrm{ft}^{3} / \mathrm{s}$.

05404750 Baraboo River tributary near Baraboo, Wis.

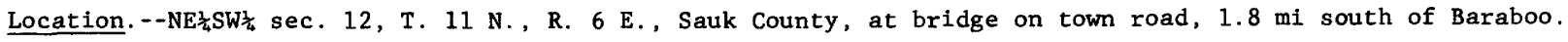
Drainage area. $--6.55 \mathrm{mi}^{2}$.

Tributary to. - -Baraboo River.

Type of site.--Miscellaneous site.

Discharge measurement. - -June $13,1973,0 \mathrm{ft}^{3} / \mathrm{s}$. 
05404760 Baraboo River tributary at Baraboo, Wis.

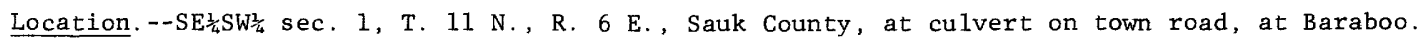

Drainage area. $--8.00 \mathrm{mi}^{2}$.

Tributary to.--Baraboo River.

Type of site.--Miscellaneous site. Discharge measurements. - June $13,1973,0.369 \mathrm{ft}^{3} / \mathrm{s} ;$ July $24,1973,0.155 \mathrm{ft} \mathrm{ft}^{3} / \mathrm{s} ;$ Oct. $25,1973,0.412 \mathrm{ft} \mathrm{t}^{3} / \mathrm{s} ;$

Low-flow frequency. $-Q_{7,2}=0.11 \mathrm{ft}^{3} / \mathrm{s}, Q_{7,10}=0.06 \mathrm{ft}^{3} / \mathrm{s}$.

Basis of estimate.--Correlated with Dell Creek near Lake Delton using 5 discharge measurements.

Accuracy. $--\mathrm{SE}_{7,10}=19$ percent (average value for basin).

05405000 Baraboo River near Baraboo, Wis.

Location. - - NW $\frac{1}{4} \mathrm{NW}_{\frac{1}{4}} \mathrm{sec} .35, \mathrm{~T} .12 \mathrm{~N}$, R. $7 \mathrm{E}$., Sauk County, on left bank $50 \mathrm{ft}$ downstream from bridge on County Trunk X, $0.3 \mathrm{mi}$ downstream from Rowley Creek and $4 \mathrm{mi}$ east of Baraboo.

Drainage area. $--609 \mathrm{mi}^{2}$.

Tributary to. - -Wisconsin River.

Type of site.--Gaging station.

Period of record.--December 1913 to March 1922. September 1942 through September 1975.

Average discharge. --40 years, $372 \mathrm{ft}^{3} / \mathrm{s}$.

Extremes. - Maximum discharge, 7,900 $\mathrm{ft}^{3} / \mathrm{s}$ Mar, 26, 1917; minimum daily discharge, $26 \mathrm{ft}^{3} / \mathrm{s} 0 \mathrm{ct} .6,1950$ (resu1t of upstream regulation).

\begin{tabular}{|l|rrrrrr|}
\hline $\begin{array}{l}\text { Period } \\
\text { of con- } \\
\text { secutive } \\
\text { days }\end{array}$ & $\begin{array}{l}\text { Magnitude and frequency of annual low flow } \\
\text { Discharge, in cubic feet per second, for } \\
\text { indicated recurrence interval, in years } \\
\text { ind }\end{array}$ \\
\hline & 2 & 5 & 10 & 20 & 50 & 100 \\
\hline 7 & 116 & 96 & 89 & 83 & 78 & 75 \\
14 & 124 & 102 & 93 & 87 & 80 & 76 \\
30 & 135 & 112 & 103 & 96 & 89 & 85 \\
60 & 152 & 124 & 112 & 104 & 95 & 89 \\
90 & 169 & 136 & 121 & 111 & 99 & 93 \\
\hline
\end{tabular}

\begin{tabular}{|lrrrrrrr|}
\hline \multicolumn{7}{|c|}{ Duration table of daily flow } \\
Discharge, in cubic feet per second, which \\
was exceeded for indicated percent of time \\
\hline $\begin{array}{l}\text { Percent } \\
\mathrm{ft}^{3} / \mathrm{s}\end{array}$ & 2,040 & 1,220 & 750 & 420 & 310 & 260 & 220 \\
\hline $\begin{array}{l}\text { Percent } \\
\mathrm{ft}^{3} / \mathrm{s}\end{array}$ & 60 & 70 & 80 & 90 & 95 & 98 & 99.9 \\
\hline
\end{tabular}

Accuracy. $--\mathrm{SE}_{7,2}=4$ percent, $\mathrm{SE}_{7,10}=4$ percent.

05405505 Rocky Run tributary at Rio, Wis.

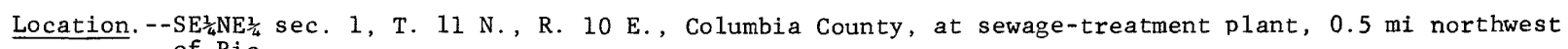
of Rio.

Drainage area. $--1.55 \mathrm{mi}^{2}$.

Tributary to. - -Wisconsin River.

Type of site.--Miscellaneous site.

Discharge measurements.--Ju1y 5, 1972,0 $\mathrm{ft}^{3} / \mathrm{s} ;$ June 25, 1973, $0 \mathrm{ft}^{3} / \mathrm{s} ; 0 \mathrm{ct} .10,1975,0 \mathrm{ft}^{3} / \mathrm{s}$.

Low-flow frequency. $-Q_{7,2}=0 \mathrm{ft}^{3} / \mathrm{s} ; Q_{7,10}=0 \mathrm{ft}^{3} / \mathrm{s}$.

Basis of estimate. --Channel observed dry on 3 visits to the site since 1972 .

Accuracy.--Not applicable.

05405560 Rocky Run near Poynette, Wis.

Location. - - $-\mathrm{SE}_{\frac{1}{4}} \mathrm{SE}^{\frac{1}{4}} \mathrm{sec} .3$, T. $11 \mathrm{~N}$, R. 9 E., Columbia County, at bridge on U.S. Highway $51,4.4 \mathrm{mi}$ north of Poynette.

Drainage area. $--32.9 \mathrm{mi}^{2}$.

Tributary to. --Wisconsin River.

Type of site.--Miscellaneous site.

Discharge measurement. --Aug. 19, 1970, $8.00 \mathrm{ft}^{3} / \mathrm{s}$. 
05405510 Rocky Run near Wyocena, Wis.

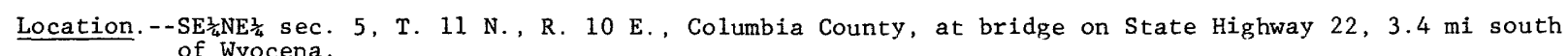
Drainage area. $--25.3 \mathrm{mi}^{2}$. Tributary to. - Wisconsin River.

Type of site. --Miscellaneous site.

Discharge measurement.--Aug. 19, 1970, $0.83 \mathrm{ft}^{3} / \mathrm{s}$.

05405570 Wisconsin River near Poynette, Wis.

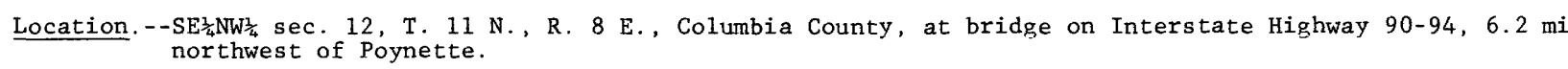

Drainage area. $--8,968 \mathrm{mi}^{2}$.

Tributary to. --Mississippi River.

Type of site.--Miscellaneous site.

Discharge measurement. - Oct. 22, 1957, 2,980 $\mathrm{ft}^{3} / \mathrm{s}$.

05405580 Rowan Creek near Poynette, Wis.

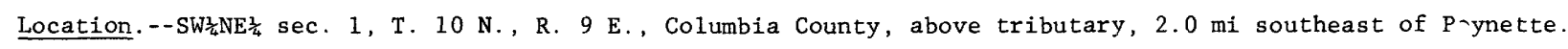
Drainage area. $--3.46 \mathrm{mi}^{2}$.

Tributary to.--Wisconsin River.

Type of site.--Miscellaneous site.

Discharge measurement.--Apr. 30, 1965, $1.5 \mathrm{ft}^{3} / \mathrm{s}$.

05405598 Rowan Creek near Poynette, Wis.

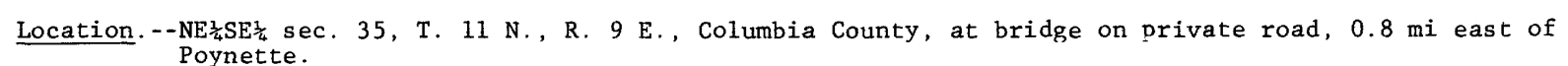
Drainage area. $--9.99 \mathrm{mi}^{2}$. Tributary to. - -Wisconsin River.

Type of site.--Miscellaneous site.

Discharge measurements. --Apr. 30, 1965, 3.3 ft $3 / \mathrm{s} ;$ Aug. $23,1965,3.3 \mathrm{ft}^{3} / \mathrm{s}$.

05405600 Rowan Creek at Poynette, Wis.

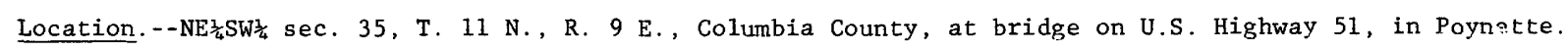
Drainage area. $--10.4 \mathrm{mi}^{2}$. Tributary to. - -Wisconsin River.

Type of site.--Low-flow partial-record station.

Minimum discharge measured. $--3.37 \mathrm{ft}^{3} / \mathrm{s}$, July $6,1965$.

Low-flow frequency. $-Q_{7,2}=3.6 \mathrm{ft}^{3} / \mathrm{s}, Q_{7,10}=2.8 \mathrm{ft}^{3} / \mathrm{s}$. Basis of estimate. - - Correlated with Black Earth Creek near Black Earth using 30 discharge measurements made
in the period 1961-75.

Accuracy. $--\mathrm{SE}_{7,10}=5$ percent.

05405650 Hinkson Creek near Poynette, Wis.

Location.-- $-\operatorname{SE}_{\frac{1}{4} \operatorname{SE}}^{\frac{1}{4}}$ sec. 21, T. 11 N., R. 9 E., Columbia County, at bridge on town road, $2.1 \mathrm{mi}$ northwest
of Poynette.

Drainage area. $--15.8 \mathrm{mi}^{2}$. Tributary to - - Rowan Creek.

Type of site.--Miscellaneous site.

Discharge measurement.--Aug. 19, 1970, $1.58 \mathrm{ft}^{3} / \mathrm{s}$. 
Location. - $-5 W_{\frac{1}{4}} \mathrm{NE}_{\frac{1}{4}} \mathrm{sec} .27$, T. $10 \mathrm{~N} ., \mathrm{R} .8 \mathrm{E}$, Columbia County, at bridge on town road in Lodi.

Drainage area. $-37.0 \mathrm{mi}^{2}$.

Tributary to.--Wisconsin River.

Type of site.--Low-flow partial-record station.

Minimum discharge measured. $--12.6 \mathrm{ft}^{3} / \mathrm{s}$, Aug. 15, 1967.

Low-flow frequency. $-Q_{7,2}=16 \mathrm{ft}^{3} / \mathrm{s}, Q_{7,10}=15 \mathrm{ft}^{3} / \mathrm{s}$.

Basis of estimate.--Correlated with Baraboo River near Baraboo using 14 discharge measurements made in the period $1962-76$.

Accuracy. $--\mathrm{SE}_{7,10}=7$ percent.

05405810 Spring Creek near Lodi, Wis.

Location. $--\mathrm{SW}_{\frac{1}{4}} \mathrm{NE} \frac{1}{4} \mathrm{sec} .21$, T. $10 \mathrm{~N}$, R. $8 \mathrm{E}$. Columbia County, at bridge on State Highway 113 , $1.6 \mathrm{mi}$ northwest of Lodi.

Drainage area. $--40.1 \mathrm{mi}^{2}$.

Tributary to. - -Wisconsin River.

Type of site.--Miscellaneous site.

Discharge measurement.--Aug. 19,1970, $19.6 \mathrm{ft}^{3} / \mathrm{s}$.

05405812 Spring Creek near Lodi, Wis.

Location. - $-\mathrm{NW} \frac{1}{4} \mathrm{NW}^{\frac{1}{4}} \mathrm{sec}$. 21 , T. $10 \mathrm{~N}$, R. 8 E., Columbia County, at bridge on State Highway 113 , 2.3 mi

Drainage area. $--45.5 \mathrm{mi}^{2}$. Tributary to. - -Wisconsin River.

Type of site.--Miscellaneous site.

Discharge measurement.--Nov. $28,1972,26.7 \mathrm{ft}^{3} / \mathrm{s}$.

05405820 Wisconsin River tributary near Merrimac, Wis.

Location. - $-\mathrm{SW} \frac{1}{4} \mathrm{SW}_{\frac{1}{4}} \mathrm{sec} .23$, T. $11 \mathrm{~N} .$, R. 7 E., Sauk County, at bridge on country road, 2.6 mi north of Merrimac.

Drainage area. $--2.82 \mathrm{mi}^{2}$.

Tributary to. - -Wisconsin River.

Type of site.--Miscellaneous site.

Discharge measurements.--June $21,1976,0.68 \mathrm{ft}^{3} / \mathrm{s} ;$ Aug. 9, 1976, 0.368 ft $3 / \mathrm{s} ; \mathrm{Aug}, 30,1976,0.515 \mathrm{ft} / \mathrm{s}$.

05405850 Wisconsin River tributary near Merrimac, Wis.

Location.-- $-\mathrm{NW}^{\frac{1}{4}} \mathrm{SE} \frac{1}{4} \mathrm{sec} .28, \mathrm{~T}, 11 \mathrm{~N} .$, R. $7 \mathrm{E}$, Sauk County, at bridge on State Highway $113,3.0$ mi northwest
of Merrimac.

Drainage area. $--3.16 \mathrm{mi}^{2}$.

Tributary to.--Wisconsin River.

Type of site.--Miscellaneous site. Discharge measurements. - June $13,1973,3.50 \mathrm{ft}^{3} / \mathrm{s} ; \mathrm{July} 24,1973,4.30 \mathrm{ft}^{3} / \mathrm{s} ; 0 \mathrm{ct} .25,1973,2.17 \mathrm{ft} / \mathrm{s} ;$

05405980 Wisconsin River tributary near Baraboo, Wis.

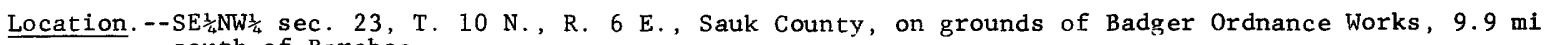
south of Baraboo.

Drainage area. $--1.77 \mathrm{mi}^{2}$.

Tributary to. - Wisconsin River.

Type of site.--Miscellaneous site.

Discharge measurement. - - oct. $9,1970,50.5 \mathrm{ft}^{3} / \mathrm{s}$. 
Table 1.--Low-flow characteristics for sites in the lower Wisconsin River basin--Continued

05406000 Wisconsin River at Prairie Du Sac, Wis.

Location. - $-\mathrm{NE} \frac{1}{4} \mathrm{NE} \frac{1}{4} \mathrm{sec} .1$, T. 9 N., R. 6 E., Sauk County, on downstream end of pier of bridge on State Highway 60 in Prairie du Sac, $1.6 \mathrm{mi}$ downstream from Prairie du Sac powerplant, $6.5 \mathrm{mi}$ upstream from Honey Creek.

Drainage area. $--9,183 \mathrm{mi}^{2}$. Tributary to.--Mississippi River.

Type of site.--Gaging station.

Period of record.--January 1946 to December 1953.

Average discharge. --7 years, 6,920 $\mathrm{ft}^{3} / \mathrm{s}$.

Extremes. - Maximum discharge, $67,700 \mathrm{ft}^{3} / \mathrm{s}$ Apr. 13, 1951; minimum daily discharge, $298 \mathrm{ft} / \mathrm{s}$ Sept. 5 and 7 , 1946.

\begin{tabular}{|c|c|c|c|c|}
\hline $\begin{array}{l}\text { Period } \\
\text { of con- } \\
\text { secutive } \\
\text { days }\end{array}$ & \multicolumn{4}{|c|}{$\begin{array}{l}\text { Magnitude and frequency of annual low flow } \\
\text { Discharge, in cubic feet per second, for } \\
\text { indicated recurrence interval, in years }\end{array}$} \\
\hline \multirow[b]{2}{*}{$\begin{array}{r}7 \\
14 \\
30 \\
60 \\
90\end{array}$} & 2 & 5 & 10 & 20 \\
\hline & $\begin{array}{l}3,050 \\
3,240 \\
3,380 \\
3,620 \\
3,770\end{array}$ & $\begin{array}{l}2,530 \\
2,690 \\
2,870 \\
3,010 \\
3,100\end{array}$ & $\begin{array}{l}2,340 \\
2,470 \\
2,700 \\
2,790 \\
2,880\end{array}$ & $\begin{array}{l}2,210 \\
2,320 \\
2,590 \\
2,640 \\
2,740\end{array}$ \\
\hline
\end{tabular}

\begin{tabular}{|lccccc|}
\hline \multicolumn{5}{|c|}{ Duration table of daily flow } \\
Discharge, in cubic feet per second, which \\
was exceeded for indicated percent of time \\
\hline $\begin{array}{l}\text { Percent } \\
\mathrm{ft}^{3} / \mathrm{s}\end{array}$ & 22,600 & 16,200 & 12,200 & 9,000 & 7,450 \\
\hline $\begin{array}{l}\text { Percent } \\
\mathrm{ft}^{3} / \mathrm{s}\end{array}$ & 40 & 50 & 60 & 70 & 80 \\
\hline $\begin{array}{l}\text { Percent } \\
\mathrm{ft}^{3} / \mathrm{s}\end{array}$ & 9,350 & 5,530 & 4,820 & 4,250 & 3,740 \\
\hline
\end{tabular}

Accuracy. $--\mathrm{SE}_{7,2}=9$ percent, $S E_{7,10}=13$ percent.

05406060 Blums Creek at Roxbury, Wis.

Location. - -SW $\frac{1}{4} S W \frac{1}{4}$ sec. 16, T. 9 N., R. 7 E., Dane County, at bridge on town road in Roxbury.

Drainage area. $--9.27 \mathrm{mi}^{2}$. Tributary to. - Wisconsin River.

Type of site.--Miscellaneous site.

Discharge measurements.--July 5, 1972, $0.028 \mathrm{ft}_{3}^{3} / \mathrm{s} ;$ June $25,1973,0.88 \mathrm{ft}^{3} / \mathrm{s} ; \mathrm{Aug} .21,1973,0.90 \mathrm{ft} / \mathrm{s} ;$ Oct. $9,1975,0.825 \mathrm{ft} / \mathrm{s}$.

Low-flow frequency. $-Q_{7,2}=0.01 \mathrm{ft}^{3} / \mathrm{s}, Q_{7,10}=<0.01 \mathrm{ft}^{3} / \mathrm{s}$.

Basis of estimate.--Correlated with Black Earth Creek at Black Earth using 4 discharge measurements.

Accuracy. $--\mathrm{SE}_{7,10}=19$ percent (average value for the basin).

05406110 Honey Creek near Plain, Wis.

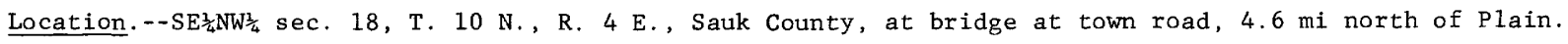
Drainage area. $--14.0 \mathrm{mi}^{2}$.

Tributary to.--Wisconsin River.

Type of site.--Miscellaneous site.

Discharge measurement.--Aug. $17,1970,1.46 \mathrm{ft}^{3} / \mathrm{s}$.

05406160 Honey Creek tributary at Plain, Wis.

Location. - $-\mathrm{SW}_{\frac{1}{4}} \mathrm{SE} \frac{1}{4} \mathrm{sec} .5, \mathrm{~T} .9 \mathrm{~N} ., \mathrm{R} .4 \mathrm{E}$. Sauk County, at sewage-treatment plant about 600 ft downstream from State Highway 23 bridge at'Plain.

Drainage area. $--6.59 \mathrm{mi}^{2}$. Tributary to.--Honey Creek.

Type of site.--Miscellaneous site.

Discharge measurements.--July 7, 1972, $0.69 \mathrm{ft}^{3} / \mathrm{s} ;$ June $26,1973,1.61 \mathrm{ft}^{3} / \mathrm{s} ; \mathrm{Aug} .22,1973,1.38 \mathrm{ft} / \mathrm{s}$; Oct. $7,1975,0.991 \mathrm{ft}^{3} / \mathrm{s}$

Low-flow frequency. $-Q_{7,2}=0.56 \mathrm{ft}^{3} / \mathrm{s}, Q_{7,10}=0.41 \mathrm{ft}^{3} / \mathrm{s}$.

Basis of estimate.--Correlated with Black Earth Creek at Black Earth using 4 discharge measurements.

Accuracy. $--\mathrm{SE}_{7,10}=19$ percent (average value for the basin). 


\section{Honey Creek near Plain, Wis.}

Location. - $-\mathrm{SE}_{\frac{1}{4}} \mathrm{NE} \frac{1}{4}$ sec. 13, T. 9 N., R. 4 E., at bridge on town road, 5.0 mi east of P1ain.

Drainage area. $--55.9 \mathrm{mi}^{2}$.

Tributary to. - -Wisconsin River.

Type of site.--Low-flow partial-record station.

Minimum discharge measured. $--7.57 \mathrm{ft}^{3} / \mathrm{s}$, Aug. 17, 1970 .

Low-flow frequency. $-Q_{7,2}=8.0 \mathrm{ft}^{3} / \mathrm{s}, n_{7,10}=6.2 \mathrm{ft}^{3} / \mathrm{s}$.

Basis of estimate.--Correlated with Black Earth Creek at Black Earth using 13 discharge measurements made in the period 1963-75.

Accuracy. $--\mathrm{SE}_{7,10}=10$ percent.

05406220 North Branch Honey Creek near Leland, Wis.

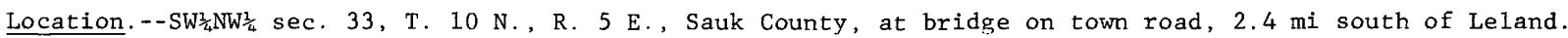
Drainage area. $--35.3 \mathrm{mi}^{2}$. Tributary to.--Honey Creek.

Type of site.--Miscellaneous site.

Discharge measurement.--Aug. $17,1970,7.95 \mathrm{ft}^{3} / \mathrm{s}$.

05406230 East Branch Honey Creek near Witwen, Wis.

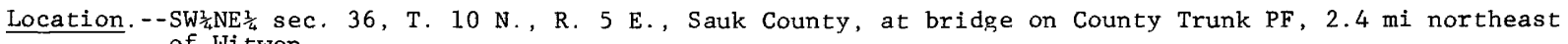
of Witwen.

Drainage area. $--23.0 \mathrm{mi}^{2}$.

Tributary to.--Honey Creek.

. Type of site.--Miscellaneous site.

Discharge measurement.--Aug. $17,1970,2.03 \mathrm{ft}^{3} / \mathrm{s}$.

05406240 Otter Creek near Prairie du Sac, Wis.

Location. - $-\mathrm{SE} \frac{1}{4} \mathrm{NW} \frac{1}{4} \mathrm{sec} .9, \mathrm{~T} .10 \mathrm{~N}$., R. 6 E., Sauk County, at bridge on County Trunk C, 6.1 mi northwest of Prairie du Sac.

Drainage area. $--17.9 \mathrm{mi}^{2}$.

Tributary to.--Honey Creek.

Type of site.--Miscellaneous site.

Discharge measurement.--Aug. 17, 1970, $0.35 \mathrm{ft}^{3} / \mathrm{s}$.

05406250 otter Creek near Sauk City, Wis.

Location.- $-\mathrm{SW}_{\frac{1}{4}} \mathrm{NE} \frac{1}{4}$ sec. 9, T. 9 N., R. $6 \mathrm{E}$. , Sauk County, at bridge on State Highway 60 , 2.8 mi west of Sauk City.

Drainage area. $--32.5 \mathrm{mi}^{2}$. Tributary to. - - Honey Creek.

Type of site.--Low-flow partial-record station.

Minimum discharge measured. $-0 \mathrm{ft}^{3} / \mathrm{s}$, July 8, 1963, Sept. 8, 1977, Sept. 6, 1967, Aug. 20, 1969, and July 9,1970

Low-flow frequency. $-Q_{7,2}=0 \mathrm{ft}^{3} / \mathrm{s}, Q_{7,10}=0 \mathrm{ft}^{3} / \mathrm{s}$.

Basis of estimate.--Correlated with Baraboo River near Baraboo using 7 discharge measurements made in the period 1963-70. 
05406300 Honey Creek near Sauk City, Wis.

Location.--SWhNE $\frac{1}{4}$ sec. 17, T. 9 N., R. 6 E., Sauk County, at bridge on State Highway 60 , 4.3 mi west of Sauk City.

Drainage area. $--187 \mathrm{mi}^{2}$.

Tributary to. --Wisconsin River.

Type of site.--Low-flow partial-record station.

Minimum discharge measured. $--29.0 \mathrm{ft}^{3} / \mathrm{s}$, Aug. 17, 1970.

Low-flow frequency. $-Q_{7,2}=34 \mathrm{ft}^{3} / \mathrm{s}, Q_{7,10}=26 \mathrm{ft}^{3} / \mathrm{s}$.

Basis of estimate.--Correlated with Baraboo River near Baraboo using 11 discharge measurements made in the period 1963-76.

Accuracy. $--\mathrm{SE}_{7,10}=10$ percent.

05406400 East Branch Blue Mounds Creek near Black Earth, Wis.

Location. - $-\mathrm{NW} \frac{1}{4} \mathrm{SW} \frac{1}{4} \mathrm{sec} .5, \mathrm{~T} .7 \mathrm{~N} ., \mathrm{R}, 6 \mathrm{E}$., Dane County, at bridge on County Trunk F, 4.2 mi southwest of Drainage area. $--29.0 \mathrm{mi}^{2}$. Tributary to. - -Wisconsin River.

Type of site.--Low-flow partial-record station.

Minimum discharge measured. $--10.3 \mathrm{ft}^{3} / \mathrm{s}, \mathrm{July} 29,1965$.

Low-flow frequency. $-Q_{7,2}=14 \mathrm{ft}^{3} / \mathrm{s}, Q_{7,10}=10 \mathrm{ft}^{3} / \mathrm{s}$.

Basis of estimate.--Correlated with Black Earth Creek at Black Earth using 16 discharge measurements made in the period 1962-70.

Accuracy. $--\mathrm{SE}_{7,10}=8$ percent.

05406430 Blue Mounds Creek near Mazomanie, Wis.

Location.-- $\begin{gathered}\text { NW } \frac{1}{4} \mathrm{NE} \frac{1}{4} \text { sec. } \\ \text { Mazomanie. }\end{gathered}$ Drainage area. $--55.0 \mathrm{mi}^{2}$. Tributary to.--Wisconsin River.

Type of site.--Miscellaneous site.

Discharge measurement.--Aug. 19, 1970, $23^{4} .1 \mathrm{ft}^{3} / \mathrm{s}$.

05406450 Black Earth Creek near Cross Plains, Wis.

Location. - $-\mathrm{NW}_{4} \mathrm{NW} \frac{3}{4} \mathrm{sec} .17, \mathrm{~T} .7 \mathrm{~N} .$, R. $8 \mathrm{E}$, Dane County, at bridge on Twin Valley Road, 3.7 mi southeast of
Cross Plains. Drainage area. $--3.32 \mathrm{mi}^{2}$. Tributary to. - - Blue Mounds Creek.

Type of site.--Miscellaneous site.

Discharge measurements.--July $10,1958,0.24 \mathrm{ft}^{3} / \mathrm{s} ;$ May $28,1964,0.51 \mathrm{ft}^{3} / \mathrm{s}$.

05406460 Black Earth Creek at Cross Plains, Wis.

Location.- - NW $\frac{1}{4} \mathrm{SE}_{\frac{1}{4}} \mathrm{sec} .3, \mathrm{~T} .7 \mathrm{~N} .$, R. 7 E., Dane County, at bridge on County Trunk P, at Cross Plains.

Drainage area. $--12.8 \mathrm{mi}^{2}$.

Tributary to.--Blue Mounds Creek.

Type of site.--Miscellaneous site.

Discharge measurement.--Aug. 17, 1970, $4.73 \mathrm{ft}^{3} / \mathrm{s}$. 
05406478 Black Earth Creek at Cross Plains, Wis.

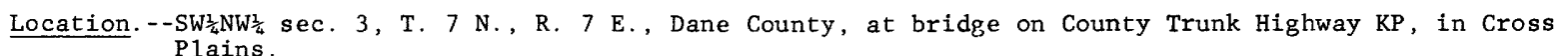

Drainage area. $--26.1 \mathrm{mi}^{2}$. Tributary to.--Blue Mounds Creek.

Type of site.--Miscellaneous site.

Minimum discharge measured. $--7.63 \mathrm{ft}^{3} / \mathrm{s}, \mathrm{Ju} 1 \mathrm{y} 7,1972$.

Low-flow frequency. $-\mathrm{Q}_{7,2}=6.0 \mathrm{ft}^{3} / \mathrm{s}, Q_{7,10}=4.3 \mathrm{ft}^{3} / \mathrm{s}$. Basis of estimate.-- Correlated with Black Earth Creek at Black Earth using 6 discharge measurements nade in
the period $1972-75$.

Accuracy. $--\mathrm{SE}_{7,10}=19$ percent (average value for basin).

05406480 Black Earth Creek at Cross Plains, Wis.

Location. - $-\mathrm{SE}_{\frac{1}{6} \mathrm{NE}}^{\frac{1}{4}} \mathrm{sec} .4, \mathrm{~T} .7 \mathrm{~N} ., \mathrm{R} .7 \mathrm{E}$, Dane County, at bridge on U.S. Highway 14, 0.1 mi downstream from sewage-disposal plant, at Cross Plains.

Drainage area. $--26.6 \mathrm{mi}^{2}$.

Tributary to.--Blue Mounds Creek.

Type of site.--Miscellaneous site.

Discharge measurements.--Nov. 19, 1963, $7.81 \mathrm{ft}^{3} / \mathrm{s} ; \mathrm{Feb} .21,1964,7.2 \mathrm{ft} / \mathrm{s}$.

05406488 Black Earth Creek tributary near Cross Plains, Wis.

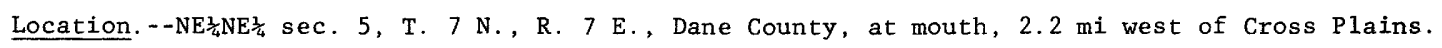

Drainage area. --Not determined.

Tributary to.--Black Earth Creek.

Type of site.--Miscellaneous site.

Discharge measurement.--Dec. $12,1973,2.61 \mathrm{ft}^{3} / \mathrm{s}$.

05406489 Black Earth Creek above mouth of Garfoot Creek near Cross Plains, Wis.

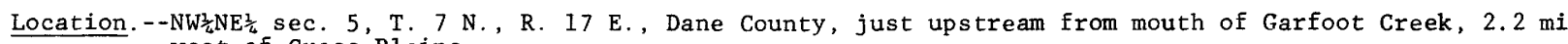
west of Cross Plains.

Drainage area. $--33.0 \mathrm{mi}^{2}$. Tributary to. - Blue Mounds Creek.

Type of site.--Miscellaneous site.

Minimum discharge measured. $--11.7 \mathrm{ft}^{3} / \mathrm{s}, \mathrm{July} 10,1958$.

Low-flow-frequency. $-\mathrm{Q}_{7,2}=12 \mathrm{ft}^{3} / \mathrm{s}, \mathrm{Q}_{7,10}=9.2 \mathrm{ft}^{3} / \mathrm{s}$.

Basis of estimate.--Correlated with Black Earth Creek at Black Earth using 6 discharge measurements made in the period 1958-73.

Accuracy. $--\mathrm{SE}_{7,10}=19$ percent (average value for basin).

05406490 Garfoot Creek near Cross Plains, Wis.

Location. - $-\mathrm{NE}_{\frac{1}{4}} \mathrm{SE}_{\frac{1}{4}} \mathrm{sec} .5, \mathrm{~T} .7 \mathrm{~N} .$, R. 7 E., Dane County, $0.5 \mathrm{mi}$ upstream from mouth near Cross Plains.

Drainage area. - - Not determined.

Tributary to.--B1ack Earth Creek.

Type of site.--Miscellaneous site.

Discharge measurement.--Dec. $12,1973,3.62 \mathrm{ft}^{3} / \mathrm{s}$. 
05406494 Black Earth Creek near Cross Plains, Wis.

Location. - - NW $\frac{1}{4} \mathrm{NE}_{\frac{1}{4}} \mathrm{sec} .5, \mathrm{~T} .7 \mathrm{~N} ., \mathrm{R} .7 \mathrm{E}$, Dane County, at bridge on town road, 2.2 mi west of Cross Plains. Drainage anea. $--39.1 \mathrm{mi}^{2}$. Tributary to.--Blue Mounds Creek.

Type of site.--Miscellaneous site.

Minimum discharge measured. $--14.4 \mathrm{ft}^{3} / \mathrm{s}, \mathrm{Feb} .19,1964$.

Low-flow frequency. $-\mathrm{Q}_{7,2}=14 \mathrm{ft}^{3} / \mathrm{s}, Q_{7,10}=11 \mathrm{ft}^{3} / \mathrm{s}$.

Basis of estimate.--Correlated with Black Earth Creek at Black Earth using 6 discharge measurements made in the period 1958-74.

Accuracy. $--\mathrm{SE}_{7,10}=19$ percent (average value for the basin).

05406497 Black Earth Creek near Black Earth, Wis.

Location. $--\mathrm{NW}_{\frac{1}{4}} \mathrm{SE}_{\frac{1}{4}} \mathrm{sec} .31$, T. $8 \mathrm{~N} .$, R. 7 E., Dane County, at bridge on town road, 2.1 mi southeast of Black Earth.

Drainage area. $--40.6 \mathrm{mi}^{2}$.

Tributary to. --Blue Mounds Creek.

Type of site.--Miscellaneous site.

Discharge measurements. --July $27,1973,38.6 \mathrm{ft}^{3} / \mathrm{s} ; \mathrm{Dec} .12,1973,34.8 \mathrm{ft}^{3} / \mathrm{s}$.

05406500 Black Earth Creek at Black Earth, Wis.

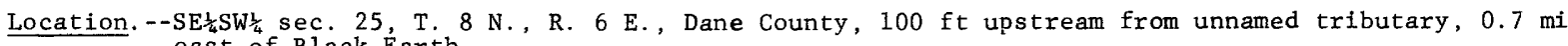
east of Black Earth.

Drainage area. $--45.6 \mathrm{mi}^{2}$.

Tributary to.--Blue Mounds Creek.

Type of site.--Gaging station.

Period of record. --February 1954 through September 1975.

Average discharge. -21 years, $31.2 \mathrm{ft}^{3} / \mathrm{s}$.

Extremes.--Maximum discharge, 1,750 $\mathrm{ft}^{3} / \mathrm{s}$ July 3, 1954; minimum discharge, 4.8 $\mathrm{ft}^{3} / \mathrm{s}$ Nov. $29,1958$.

\begin{tabular}{|l|rrrrrrr|}
\hline $\begin{array}{l}\text { Period } \\
\text { of con- } \\
\text { secutive } \\
\text { days }\end{array}$ & $\begin{array}{l}\text { Magnitude and frequency of annual low flow } \\
\text { Discharge, in cubic feet per second, for } \\
\text { indicated recurrence interval, in years } \\
\text { ind }\end{array}$ \\
\hline & 2 & 5 & 10 & 20 & 50 & 100 \\
\cline { 2 - 7 } & 17 & 15 & 13 & 13 & 12 & 12 \\
7 & 18 & 15 & 14 & 13 & 12 & 12 \\
30 & 19 & 16 & 15 & 14 & 13 & 13 \\
60 & 20 & 17 & 15 & 14 & 13 & 13 \\
90 & 21 & 17 & 16 & 15 & 14 & 14 \\
\hline
\end{tabular}

\begin{tabular}{|c|c|c|c|c|c|c|c|}
\hline \multicolumn{8}{|c|}{$\begin{array}{l}\text { Duration table of daily flow } \\
\text { Discharge, in cubic feet per second, whict } \\
\text { was exceeded for indicated percent of time }\end{array}$} \\
\hline \multirow{2}{*}{$\begin{array}{l}\text { Percent } \\
\mathrm{ft}^{3} / \mathrm{s}\end{array}$} & 2 & 5 & 10 & 20 & 30 & 40 & $5 C$ \\
\hline & 95 & 60 & 47 & 37 & 32 & 28 & $2 \epsilon$ \\
\hline \multirow{2}{*}{$\begin{array}{l}\text { Percent } \\
\mathrm{ft}^{3} / \mathrm{s}\end{array}$} & 60 & 70 & 80 & 90 & 95 & 98 & 99.9 \\
\hline & 23 & 21 & 20 & 17 & 16 & 15 & 13 \\
\hline
\end{tabular}

Accuracy. $--\mathrm{SE}_{7,2}=6$ percent, $\mathrm{SE}_{7,10}=7$ percent.

05406508 Black Earth Creek at Black Earth, Wis.

Location. - - $\mathrm{SE}_{\frac{1}{4} \mathrm{NW}} \mathrm{sec} \mathrm{sec} 26, \mathrm{~T} .8 \mathrm{~N} ., \mathrm{R} .6 \mathrm{E}$, Dane County, at bridge on U.S. Highway 14 and State Highway 78. $200 \mathrm{ft}$ upstream of sewage-treatment plant, at Black Earth.

Drainage area. $--48.3 \mathrm{mi}^{2}$.

Tributary to.--Blue Mounds Creek.

Type of site.--Miscellaneous site.

Discharge measurement.--July $27,1973,43.2 \mathrm{ft}^{3} / \mathrm{s}$. 
05406510 Black Earth Creek at mouth of Vermont Creek at Black Earth, Wis.

Location. - $-\mathrm{SW} \frac{1}{4} \mathrm{NW} \frac{1}{4} \mathrm{sec} .26, T .8 \mathrm{~N}$, R. $6 \mathrm{E}$., Dane County, just upstream from mouth of Vermont Creek, at west edge of Black Earth.

Drainage area. $--48.4 \mathrm{mi}^{2}$. Tributary to.--Blue Mounds Creek.

Type of site.--Miscellaneous site.

Discharge measurements.--July $27,1973,43.2 \mathrm{ft}^{3} / \mathrm{s} ;$ Dec. 12, 1973, 41.6 $\mathrm{ft}^{3} / \mathrm{s}$.

05406515 Vermont Creek at mouth at Black Earth, Wis.

Location.--SW $\frac{1}{4} \mathrm{NW}_{\frac{1}{6}}$ sec. 26, T. 8 N., R. 6 E., Dane County, at Chicago, Milwaukee, St. Paul, and Pacific Railroad bridge in Dane County, just west of Black Earth.

Drainage area. $--14.9 \mathrm{mi}^{2}$.

Tributary to. --Black Earth Creek.

Type of site, --Miscellaneous site.

Discharge measurements. --July $27,1973,10.3 \mathrm{ft}^{3} / \mathrm{s} ;$ Dec. 12, 1973, $7.19 \mathrm{ft}^{3} / \mathrm{s}$.

05406516 Black Earth Creek near Mazomanie, Wis.

Location. - $-\mathrm{SW}_{\frac{1}{4}} \mathrm{NW} \frac{1}{4} \mathrm{sec} .22$, T. $8 \mathrm{~N}$, R. $6 \mathrm{E}$., Dane County, at bridge on town road, $1.7 \mathrm{mi}$ southeast of Mazomanie.

Drainage area. $--68.9 \mathrm{mi}^{2}$.

Tributary to. --Blue Mounds Creek.

Type of site.--Miscellaneous site.

Discharge measurements.--July $27,1973,59.3 \mathrm{ft}^{3} / \mathrm{s} ;$ Dec. 12, 1973, 49.0 $\mathrm{ft}^{3} / \mathrm{s}$.

05406518 Black Earth Creek above Halfway Prairie Creek at Mazomanie, Wis.

Location. - $-\mathrm{NW}_{\frac{1}{4}} \mathrm{NE}_{\frac{1}{4}} \mathrm{sec}, 16, \mathrm{~T}, 8 \mathrm{~N} ., \mathrm{R}, 6 \mathrm{E}$, Dane County, just upstream from mouth of Halfway Prairie Creek, at Mazomanie.

Drainage area. $--70.9 \mathrm{mi}^{2}$.

Tributary to.--Blue Mounds Creek.

Type of site.--Misrellaneous site.

Discharge measurements.--July $27,1973,61.1 \mathrm{ft}^{3} / \mathrm{s} ; \mathrm{Dec} .12,1973,47.6 \mathrm{ft}^{3} / \mathrm{s}$.

05406520 Halfway Prairie Creek near Mazomanie, Wis.

Location. - $-\mathrm{NE}_{\frac{1}{4} \mathrm{SE}} \frac{1}{4} \mathrm{sec} .10, \mathrm{~T} .8 \mathrm{~N} .$, R. 6 E., Dane County, at bridge on State Highways 19 and 78 , 1.8 mi east of Mazomanie.

Drainage area. $--16.1 \mathrm{mi}^{2}$. $\quad$ Tributary to. - -Black Earth Creek.

Type of site. - Miscellaneous site.

Discharge measurement.--Aug. 17, 1970, $1.60 \mathrm{ft}^{3} / \mathrm{s}$.

05406535 Halfway Prairie Creek at mouth in Mazomanie, Wis.

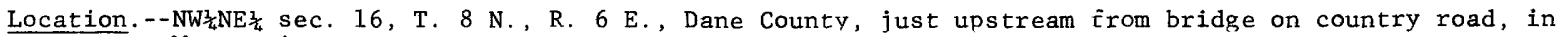
Mazomanie.

Drainage area. $--27.5 \mathrm{mi}^{2}$. Tributary to.--Black Earth Creek.

Type of site.--Miscellaneous site.

Discharge measurement. --Dec. 12, 1973, $7.99 \mathrm{ft}^{3} / \mathrm{s}$. 
05406537 Black Earth Creek at Mazomanie, Wis.

Location. - $-\mathrm{SW} \frac{1}{4} \mathrm{SE} \frac{1}{4}$ sec. 9, T. 8 N., R. $6 \mathrm{E}$., Dane County, at bridge on Hudson Road, $0.4 \mathrm{mi}$ east of County Trunk $\mathrm{Y}$ at village limits, at Mazomanie.

Drainage area. $--98.3 \mathrm{mi}^{2}$. Tributary to.--Blue Mounds Creek.

Type of site.--Miscellaneous site.

Discharge measurements.--Apr. 17, 1964, 31.0 $\mathrm{ft}_{3}^{3} / \mathrm{s} ; \mathrm{July} 27,1964,18.3 \mathrm{ft}^{3} / \mathrm{s} ; \mathrm{Nov} .24,1964,21.7 \mathrm{ft} / \mathrm{s}$; Nov. $15,1966,20.0 \mathrm{ft} / \mathrm{s}$

Low-flow frequency. $-Q_{7,2}=22 \mathrm{ft}^{3} / \mathrm{s}, \mathrm{Q}_{7,10}=15 \mathrm{ft}^{3} / \mathrm{s}$.

Basis of estimate.--Correlated with Black Earth Creek at Black Earth using 4 discharge measurements.

Accuracy. $--\mathrm{SE}_{7,10}=19$ percent (average value for basin).

05406540 Black Earth Creek at Mazomanie, Wis

Location. - -SW $\frac{1}{4}$ SW $\frac{3}{4}$ sec. 9, T. 8 N., R. 6 E., Dane County, at bridge on Bridge Street, in Mazomanie.

Drainage area. $--99.0 \mathrm{mi}^{2}$. Tributary to. - Blue Mounds Creek.

Type of site.--Miscellaneous site.

Minimum discharge measured. $--28.0 \mathrm{ft}^{3} / \mathrm{s}, \mathrm{July} 7,1972$.

Low-flow frequency. $-\mathrm{Q}_{7,2}=22 \mathrm{ft}^{3} / \mathrm{s}, Q_{7,10}=15 \mathrm{ft}^{3} / \mathrm{s}$.

Basis of estimate.--Correlated with Black Earth Creek at Black Earth using 6 discharge measurements made in the period 1972-75.

Accuracy. $--\mathrm{SE}_{7,10}=19$ percent (average value for the basin).

05406544 Black Earth Creek near Mazomanie, Wis.

Location. $-\mathrm{SE}_{\frac{1}{4}} \mathrm{SE}_{\frac{1}{4}} \mathrm{sec} .7$, T. $8 \mathrm{~N}$, R. 6 E., Dane County, at bridge on town road, $1.2 \mathrm{mi}$ west of Mazomanie. Drainage area. $--100 \mathrm{mi}^{2}$ Tributary to.--Blue Mounds Creek.

Type of site.--Miscellaneous site.

Discharge measurements.--July $27,1973,61.7 \mathrm{ft}_{3}^{3} / \mathrm{s} ;$ Dec. 12, 1973, 53.7 $\mathrm{ft}_{3}^{3} / \mathrm{s} ; \mathrm{June} 15,1976,37.8 \mathrm{ft} / \mathrm{s}$; Mar. 23, 1976, $74.6 \mathrm{ft}^{3} / \mathrm{s} ;$ June $10,1976,33.6 \mathrm{ft}^{3} / \mathrm{s}$.

Low-flow frequency. $-Q_{7,2}=15 \mathrm{ft}^{3} / \mathrm{s}, Q_{7,10}=9.8 \mathrm{ft}^{3} / \mathrm{s}$.

Basis of estimate.--Correlated with Black Earth Creek at Black Earth using 5 discharge measurements.

Accuracy. $--\mathrm{SE}_{7,10}=19$ percent (average value for the basin).

05406546 Black Earth Creek near Mazomanie, Wis.

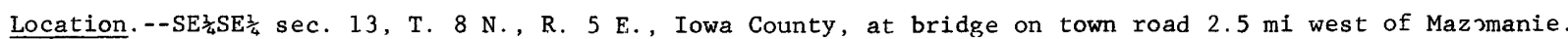

Drainage area. $--104 \mathrm{mi}^{2}$.

Tributary to.--Blue Mounds Creek.

Type of site.--Miscellaneous site.

Minimum discharge measured. $--5.98 \mathrm{ft}^{3} / \mathrm{s}$, July $30,1965$.

Low-flow frequency. $-Q_{7,2}=13 \mathrm{ft}^{3} / \mathrm{s}, Q_{7,10}=7.4 \mathrm{ft}^{3} / \mathrm{s}$.

Basis of estimate.--Correlated with Black Earth Creek at Black Earth using 7 discharge measurements made in the period $1964-73$.

Accuracy. $--\mathrm{SE}_{7,10}=19$ percent (average value for the basin). 
05406548 Black Earth Creek near Mazomanie, Wis.

Location. - - $\mathrm{SE}_{\frac{1}{4}} \mathrm{SE}_{\frac{1}{4}} \mathrm{sec} .14$, T. $8 \mathrm{~N} .$, R. $5 \mathrm{E}$., Iowa County, at bridge on town road, 3.6 mi west of Mazomanie. Drainage area. $--105 \mathrm{mi}^{2}$. Tributary to.--Blue Mounds Creek.

Type of site.--Miscellaneous site.

Discharge measurements. --July 27, 1973, 61.3 $\mathrm{ft}_{3}^{3} / \mathrm{s} ;$ Dec. 12, 1973, $66.5 \mathrm{ft}^{3} / \mathrm{s} ;$ Dec. $12,1973,50.6 \mathrm{ft}^{3} / \mathrm{s}$;

Low-flow frequency. $-Q_{7,2}=12 \mathrm{ft}^{3} / \mathrm{s}, Q_{7,10}=6.8 \mathrm{ft}^{3} / \mathrm{s}$

Basis of estimate.--Correlated with Black Earth Creek at Black Earth using 4 discharge measurements.

Accuracy. $--\mathrm{SE}_{7,10}=19$ percent (average value for the basin).

05406573 Trout Creek at confluence with Arneson Creek near Barneveld, Wis.

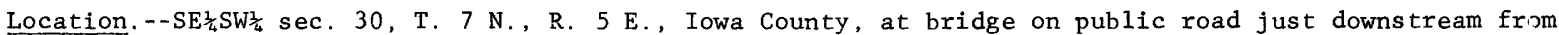
Arneson Creek, $3.5 \mathrm{mi}$ northwest of Barneveld.

Drainage area. $--8.37 \mathrm{mi}^{2}$.

Tributary to.--Mill Creek.

Type of site.--Gaging station.

Minimum discharge measured. $--4.69 \mathrm{ft}^{3} / \mathrm{s}$, Sept. 23, 1971.

Low-flow frequency. $--Q_{7,2}=3.6 \mathrm{ft}^{3} / \mathrm{s}, Q_{7,10}=2.9 \mathrm{ft}^{3} / \mathrm{s}$.

Basis of estimate. - Correlated with Black Earth Creek at Black Earth using 11 discharge measurements made during the period 1971-76.

Accuracy.--SE $7,10=13$ percent.

05406574 Trout Creek at Twin Parks Watershed, Dam 8, near Barneveld, Wis.

Location. - - SE $\frac{1}{4} S W \frac{1}{4}$ sec. 19, T. 7 N., R. 5 E., Iowa County, at west edge of Twin Parks Watershed Detent:on Reservoir Site No. 8, $400 \mathrm{ft}$ upstream from mouth of Duesler Creek, 4.2 mi northwest of Barneveld.

Drainage area. $--9.02 \mathrm{mi}^{2}$.

Tributary to. --Mill Creek.

Type of site.--Gaging station.

Minimum discharge measured. $--6.35 \mathrm{ft}^{3} / \mathrm{s}$, Jan. 14, 1976.

Low-flow frequency. $--Q_{7,2}=4.6 \mathrm{ft}^{3} / \mathrm{s}, Q_{7,10}=3.8 \mathrm{ft}^{3} / \mathrm{s}$.

Basis of estimate.--Correlated with Black Earth Creek at Black Earth using 10 discharge measurements made during the period 1975-76.

Accuracy. $-\mathrm{SE}_{7,10}=10$ percent.

05406575 Trout Creek at County Highway $\mathrm{T}$ near Barneveld, Wis.

Location.--NW $\frac{1}{4}$ SW $\frac{1}{2}$ sec. 19, T. 7 N., R. 5 E., Iowa County, on left bank at old bridge site, $400 \mathrm{ft}$ upstream from County Trunk Highway $T, 0.2$ mi downstream from mouth of Duesler Creek, $0.3 \mathrm{mi}$ downstream from Twin Parks Dam 8 (Detention Reservoir), 4.5 mi northwest of Barneveld.

Drainage area. $--12.1 \mathrm{mi}^{2}$.

Tributary to. --Mill Creek.

Type of site.--Gaging station.

Minimum discharge measured. $--7.82 \mathrm{ft}^{3} / \mathrm{s}$, Jan. 14, 1976 and Feb. 3, 1976.

Low-flow frequency. $-Q_{7,2}=5.5 \mathrm{ft}^{3} / \mathrm{s}, Q_{7,10}=4.4 \mathrm{ft}^{3} / \mathrm{s}$.

Basis of estimate.--Correlated with Black Earth Creek at Black Earth using 11 discharge measurements made during the period 1971-76.

Accuracy. $--\mathrm{SE}_{7,10}=11$ percent. 
05406577 Trout Creek near Ridgeway, Wis.

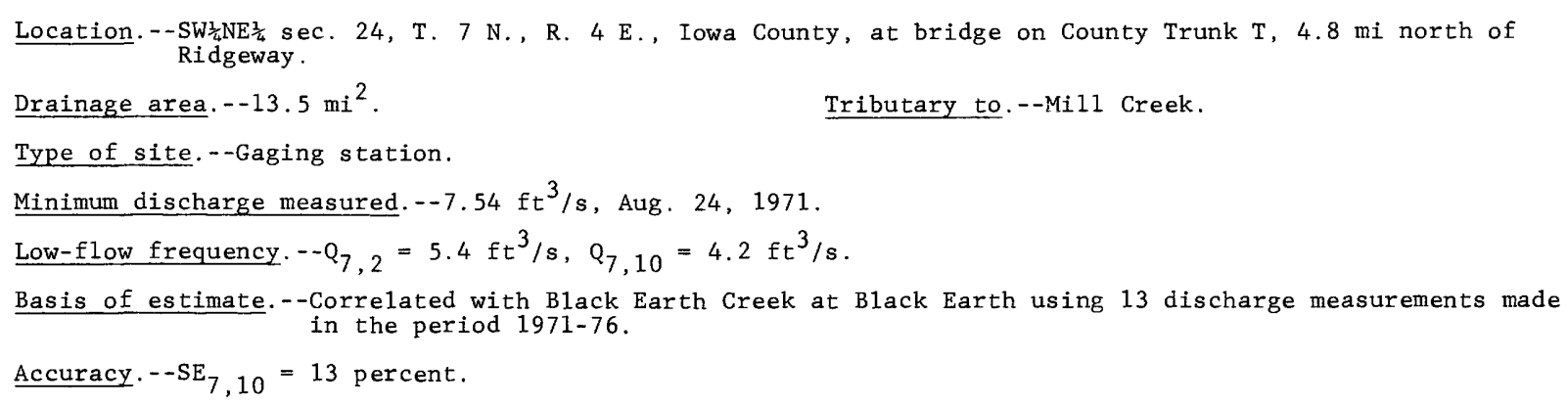

Type of site.--Gaging station.

Minimum discharge measured. $--7.54 \mathrm{ft}^{3} / \mathrm{s}$, Aug. 24, 1971.

Low-flow frequency. $-Q_{7,2}=5.4 \mathrm{ft}^{3} / \mathrm{s}, Q_{7,10}=4.2 \mathrm{ft} / \mathrm{s}$.

Basis of estimate.--Correlated with B1ack Earth Creek at Black Earth using 13 discharge measurements made in the period 1971-76.

Accuracy. $--\mathrm{SE}_{7,10}=13$ percent.

05406580 Mi11 Creek near Barneve1d, Wis.

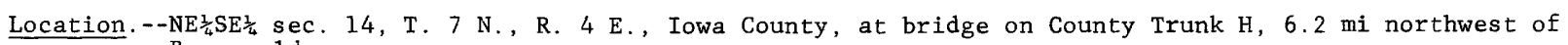
Barneve1d.

Drainage area. $--66.5 \mathrm{mi}^{2}$.

Tributary to. --Wisconsin River.

Type of site.--Misce11aneous site.

Discharge measurement.--Aug. 18, 1970, $10.2 \mathrm{ft}^{3} / \mathrm{s}$.

05406593 Mi11 Creek near Arena, Wis.

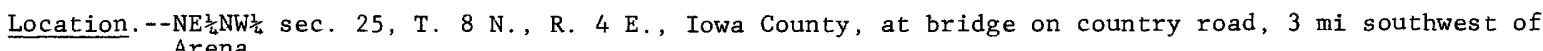
Arena.

Drainage area. $--90.5 \mathrm{mi}^{2}$.

Tributary to. --Wisconsin River.

Type of site.--Miscellaneous site.

Discharge measurement. --July $27,1964,29.5 \mathrm{ft}^{3} / \mathrm{s}$.

05406600 Mı11 Creek near Spring Green, Wis.

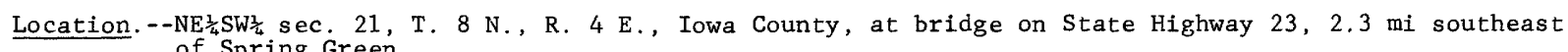
Drainage area. $--96.8 \mathrm{mi}^{2}$ Tributary to. --Wisconsin River.

Type of site. --Low-flow partial-record station.

Minimum discharge measured. $--30.9 \mathrm{ft}^{3} / \mathrm{s}$, Ju1y $30,1965$.

Low-flow frequency. $-Q_{7,2}=36 \mathrm{ft}^{3} / \mathrm{s}, Q_{7,10}=31 \mathrm{ft}^{3} / \mathrm{s}$.

Basis of estimate. - - Correlated with Black Earth Creek at Black Earth using 11 discharge measurements made in the period 1963-76.

Accuracy. $--\mathrm{SE}_{7,10}=15$ percent.

05406605 Lowery Creek near Spring Green, Wis .

Location.--SE $\frac{1}{4} \mathrm{SW}_{\frac{1}{4}} \mathrm{sec} .30$, T. 8 N., R. 4 E., Iowa County, at bridge on County Trunk T, $3.0 \mathrm{mi}$ south of Drainage area. $--8.76 \mathrm{mi}^{2}$. Tributary to. --Wisconsin River.

Type of site.--Miscellaneous site.

Discharge measurement.--Aug. 19, 1970, $2.84 \mathrm{ft}^{3} / \mathrm{s}$. 
05406615 Rush Creek near Spring Green, Wis.

Location. - $-\mathrm{SW}_{\frac{1}{4}} \mathrm{NW}_{\frac{1}{4}} \mathrm{sec} .35, \mathrm{~T} .8 \mathrm{~N}$., R. $3 \mathrm{E}$., Iowa County, at bridge on State Highway 23 , 4.2 mi southwest of Spring Green.

Drainage area $--13.9 \mathrm{mi}^{2}$

Tributary to. --Sneed Creek.

Type of site.--Miscellaneous site.

Discharge measurements. --Aug. 19, 1970, 3.18 ft $\mathrm{ft}^{3} / \mathrm{s} ; 0 \mathrm{ct} .7,1975,0,429 \mathrm{ft}^{3} / \mathrm{s}$.

05406640 Otter Creek near Highland, Wis.

Location.--SW $\frac{1}{4} \mathrm{NE} \frac{1}{4} \mathrm{sec} .5, \mathrm{~T} .6 \mathrm{~N}$, R. $2 \mathrm{E}$., Iowa County, at bridge on country road, $5.4 \mathrm{mi}$ east of Highland.

Drainage area. $-16.8 \mathrm{mi}^{2}$.

Tributary to.--Wisconsin River.

Type of site.--Gaging station.

Period of record.--May 1968 to June 1969, August 1970 to September 1975.

Average discharge. -5 years, $10.7 \mathrm{ft}^{3} / \mathrm{s}$.

Regulation.--Flow regulated by Black Hawk Lake Reservoir $0.3 \mathrm{mi}$ upstream since August 1970.

Extremes.--Maximum discharge, $148 \mathrm{ft}^{3} / \mathrm{s}$ Mar. 17, 1969, minimum discharge $0.07 \mathrm{ft} / \mathrm{s}$ Aug. $27,1970 \mathrm{resu} 1 \mathrm{t}$ of shutdown of dam outlet.

Low-flow frequency. $--Q_{7,2}=2.9 \mathrm{ft}^{3} / \mathrm{s}, Q_{7,10}=2.1 \mathrm{ft}^{3} / \mathrm{s}$.

Basis of estimate.--Correlated with Black Earth Creek at Black Earth using, 13 discharge measurements made during the period 1064-69.

Accuracy. $-\mathrm{SE}_{7,10}=18$ percent.

05406641 Norvison Branch near Highland, Wis.

Location. - $-\mathrm{NW} \frac{1}{4} \mathrm{NE} \frac{1}{4}$ sec. 5, T. $6 \mathrm{~N}$. R. $2 \mathrm{E}$., Iowa County, at Countv Trunk P, above confluence with Otter Creek, $5.3 \mathrm{mi}$ east of Highland.

Drainage area. $--1.04 \mathrm{mi}^{2}$.

Tributary to.--Otter Creek.

Type of site.--Miscellaneous site.

Minimum discharge measured.--1.21 $\mathrm{ft}^{3} / \mathrm{s}$, June 19, 1968.

Low-flow frequency. $-Q_{7,2}=1.6 \mathrm{ft}^{3} / \mathrm{s}, Q_{7,10}=1.3 \mathrm{ft}^{3} / \mathrm{s}$.

Basis of estimate.--Correlated with Black Earth Creek at Black Earth using 10 discharge measurements made during the period 1968-70.

Accuracy. $--\mathrm{SE}_{7,10}=14$ percent.

05406643 Flint Creek near clyde, Wis

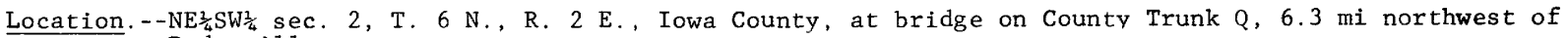
Dodgeville.

Drainage area. $--16.7 \mathrm{mi}^{2}$. Tributary to.--Otter Creek.

Type of site.--Miscellaneous site.

Discharge measurements.--Aug. 18, 1970, 2.30 ft $3 / \mathrm{s} ;$ Aug. 13, 1964, $2.5 \mathrm{ft}^{3} / \mathrm{s}$

05406646 otter Creek near Highland, Wis.

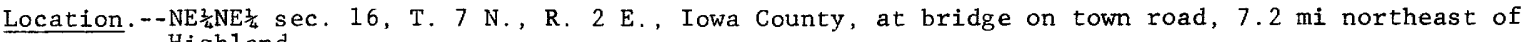
Highland.

Drainage area. $--60.5 \mathrm{mi}^{2}$.

Tributary to.--Wisconsin River.

Type of site.--Miscellaneous site.

Discharge measurements.--Aug. 14, 1964, $12.6 \mathrm{ft}^{3} / \mathrm{s} ;$ Aug. 19, 1970, $18.1 \mathrm{ft}^{3} / \mathrm{s}$. 
Table 1.--Low-flow characteristics for sites in the lower Wisconsin River basin--Continued

05406648 Otter Creek tributary near Highland, Wis.

Location.- - NW $\frac{1}{4}$ NE $\frac{1}{4} \mathrm{sec} .16$, T. 7 N., R. 2 E., Iowa County, at bridge on County Trunk II, 7.0 mi northeast of Highland.

Drainage area. $--19.8 \mathrm{mi}^{2}$. Tributary to.--Otter Creek.

Type of site.-Miscellaneous site.

Discharge measurements.--Aug. 14, 1964, $5.0 \mathrm{ft}^{3} / \mathrm{s} ;$ Aug. 19, 1970, $5.38 \mathrm{ft}^{3} / \mathrm{s}$.

05406650 Otter Creek near Clyde, Wis.

Location.--SE $\frac{1}{4} \mathrm{NE}_{\frac{1}{4}} \mathrm{sec} .2, \mathrm{~T} .7 \mathrm{~N} ., \mathrm{R} .2 \mathrm{E}$, Iowa County, at bridge on County Trunk I, 0.5 mi south of Clyde. Drainage area. $--95.6 \mathrm{mi}^{2}$. Tributary to. - -Wisconsin River.

Type of site.--Low-flow partial-record station.

Minimum discharge measured. $-21.5 \mathrm{ft}^{3} / \mathrm{s}$, Sept. 6, 1967.

Low-flow frequency. $--Q_{7,2}=25 \mathrm{ft}^{3} / \mathrm{s}, Q_{7,10}=18 \mathrm{ft}^{3} / \mathrm{s}$.

Basis of estimate.--Correlated with Black Earth Creek at Black Earth using 11 discharge measurements made in the period 1963-76.

Accuracy. $--\mathrm{SE}_{7,10}=10$ percent.

05406657 Otter Creek near Clyde, Wis.

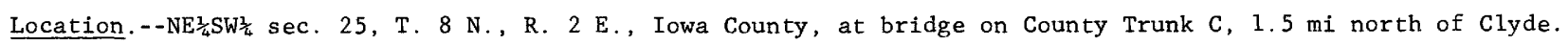
Drainage area. $--107 \mathrm{mi}^{2}$. Tributary to.--Wisconsin River.

Type of site.--Miscellaneous site.

Discharge measurement.--July $27,1964,26.4 \mathrm{ft}^{3} / \mathrm{s}$.

05406680 Bear Creek near Plain, Wis.

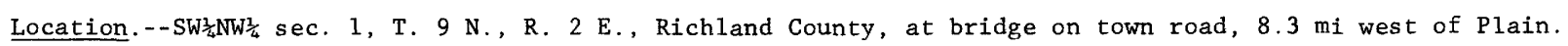

Drainage area. $--37.5 \mathrm{mi}^{2}$. Tributary to. - -Wisconsin River.

Type of site.--Miscellaneous site.

Discharge measurement.--Aug. $17,1970,13.7 \mathrm{ft}^{3} / \mathrm{s}$.

05406700 Bear Creek near Lone Rock, Wis.

Location.--SE $\frac{1}{4} N W \frac{1}{4}$ sec. 35, T. 9 N., R. 2 E., Richland County, at bridge on County Trunk JJ, 2.5 mi northwest of Lone Rock.

Drainage area. $--65.5 \mathrm{mi}^{2}$. Tributary to.--Wisconsin River.

Type of site.--Low-flow partial-record station.

Minimum discharge measured. $--15.2 \mathrm{ft}^{3} / \mathrm{s}$, July 30, 1965.

Low-flow frequency. $-Q_{7,2}=20 \mathrm{ft}^{3} / \mathrm{s}, Q_{7,10}=17 \mathrm{ft}^{3} / \mathrm{s}$.

Basis of estimate.--Correlated with Black Earth Creek at Black Earth using 11 discharge measurements made in the period 1963-76.

Accuracy. $--\mathrm{SE}_{7,10}=9$ percent.

05406704 Pine River near Yuba, Wis.

Location. -
Yuba. Drainage area. $--9.64 \mathrm{mi}^{2}$. Tributary to. - -Wisconsin River.

Type of site.--Miscellaneous site.

Discharge measurements.--0ct. $17,1972,4.36 \mathrm{ft}^{3} / \mathrm{s} ;$ June $3,1974,6.09 \mathrm{ft}^{3} / \mathrm{s}$. 
Table 1.--Low-flow characteristics for sites in the lower Wisconsin River basin--Continued

05406706 Pine River tributary No. 1 near Yuba, Wis.

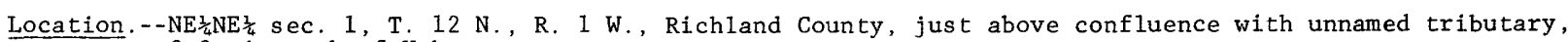
$0.9 \mathrm{mi}$ north of Yuba.

Drainage area. $--7.37 \mathrm{mi}^{2}$. Tributary to. - -Pine River.

Type of site.--Miscellaneous site.

Discharge measurements.--Oct. 17, 1972, $3.85 \mathrm{ft}^{3} / \mathrm{s}$; June $3,1974,4.52 \mathrm{ft} / \mathrm{s}$.

05406708 Tributary to Pine River tributary near Yuba, Wis.

Location. - $-\mathrm{NE}_{\frac{1}{4}} \mathrm{NE}_{\frac{1}{4}} \mathrm{sec} .1, \mathrm{~T} .12 \mathrm{~N} ., \mathrm{R} .1 \mathrm{~W} .$, Richland County, at mouth, 0.9 mi north of Yuba.

Drainage area. $--8.13 \mathrm{mi}^{2}$. Tributary to.--Pine River tributary No. 1

Type of site.--Miscellaneous site.

Discharge measurements.--Oct. 17, 1972, 3.94 $\mathrm{ft}^{3} / \mathrm{s} ;$ June $3,1974,4.80 \mathrm{ft}^{3} / \mathrm{s}$.

05406710 Pine River at Yuba, Wis.

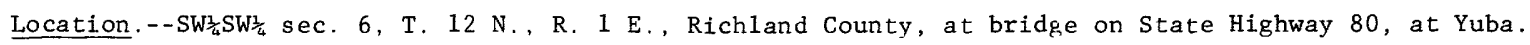

Drainage area. $--27.2 \mathrm{mi}^{2}$. Tributary to.--Wisconsin River.

Type of site.--Miscellaneous site.

Minimum discharge measured. $--5.27 \mathrm{ft}^{3} / \mathrm{s}$, July 6, 1972.

Low-flow frequency. $--Q_{7,2}=3.7 \mathrm{ft}^{3} / \mathrm{s}, Q_{7,10}=2.5 \mathrm{ft}^{3} / \mathrm{s}$. Basis of estimate. - - Correlated with Kickapoo River at LaFarge using 6 discharge measurements made in the

Accuracy. $-\mathrm{SE}_{7,10}=19$ percent (average value for the basin).

05406711 Indian Creek near Yuba, Wis.

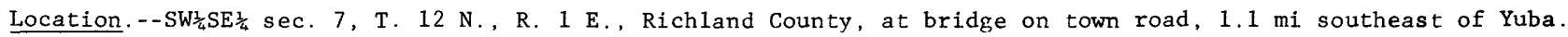
Drainage area. $--2.59 \mathrm{mi}^{2}$. $\quad$ Tributary to. - -Pine River.

Type of site.--Miscellaneous site.

Discharge measurements. --Oct. 17, 1972, $1.02 \mathrm{ft}^{3} / \mathrm{s} ;$ June $3,1974,1.62 \mathrm{ft}^{3} / \mathrm{s}$.

05406712 Pine River near Yuba, Wis.

Location. - - NW $\frac{1}{4} \mathrm{NE}_{\frac{1}{4}} \mathrm{sec} .18, \mathrm{~T}, 12 \mathrm{~N} ., \mathrm{R}, 1 \mathrm{E}$. , Richland County, at bridge on State Highway $80,1.4 \mathrm{mi}$ s 3 utheast Drainage area. $--32.7 \mathrm{mi}^{2}$. Tributary to. --Wisconsin River.

Type of site.--Miscellaneous site.

Discharge measurement.--Aug. $19,1970,6.69 \mathrm{ft}^{3} / \mathrm{s}$.

05406714 Pine River near Hub City, Wis.

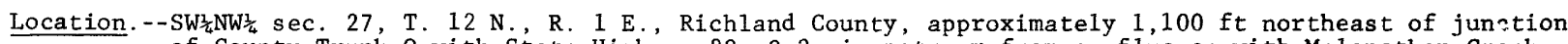
of County Trunk $O$ with State Highway $80,0.2 \mathrm{mi}$ upstream from confluence with Melancthon $\mathrm{Cr} \approx e k$, 1.2 mi north of Hub City.

Drainage area. $--41.1 \mathrm{mi}^{2}$.

Tributary to. - -Wisconsin River.

Type of site.--Miscellaneous site.

Discharge measurements.--June 3, 1974, $31.79 \mathrm{ft}^{3} / \mathrm{s}$; Sept. 9, 1974, $25.3 \mathrm{ft} / \mathrm{s}$; May 15, $1975,27.9 \mathrm{ft}^{3} / \mathrm{s}$. 
05406715 Melancthon Creek near Yuba, Wis.

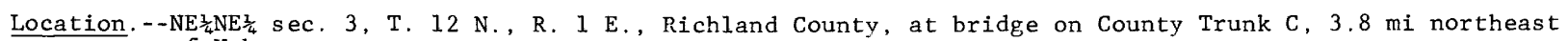
of Yuba.

Drainage area. - - $1.93 \mathrm{mi}^{2}$. Tributary to.--Pine River.

Type of site.--Miscellaneous site.

Discharge measurements.--June 3, 1974, $1.53 \mathrm{ft}^{3} / \mathrm{s} ;$ Sept. 9, 1974, 1.16 ft $\mathrm{fts}^{3}$ May $14,1975,1.62 \mathrm{ft} / \mathrm{s}$.

05406716 Melancthon Creek near Yuba, Wis

Location. - $-\mathrm{NE} \frac{1}{4} \mathrm{NE} \frac{1}{4} \mathrm{sec} .10, \mathrm{~T} .12 \mathrm{~N} .$, R. 1 E., Richland County, approximately 500 ft north of private farm drive, $0.3 \mathrm{mi}$ upstream from mouth of Grinsell Branch, $36 \mathrm{mi}$ east of Yuba.

Drainage area. $--4.66 \mathrm{mi}^{2}$. Tributary to.--Pine River.

Type of site.--Miscellaneous site.

Discharge measurements.--June $3,1974,3.98 \mathrm{ft}^{3} / \mathrm{s} ;$ Sept., 9, 1974, 2.97 $\mathrm{ft}^{3} / \mathrm{s} ; \mathrm{May} 14,1975,3.09 \mathrm{ft} / \mathrm{s}$;

Low-flow frequency. $-Q_{7,2}=2.3 \mathrm{ft}^{3} / \mathrm{s}, Q_{7,10}=2.0 \mathrm{ft}^{3} / \mathrm{s}$.

Basis of estimate.--Correlated with Baraboo using 4 discharge measurements.

Accuracy. $--\mathrm{SE}_{7,10}=19$ percent (average value for basin).

05406717 Melancthon Creek near Hub City, Wis.

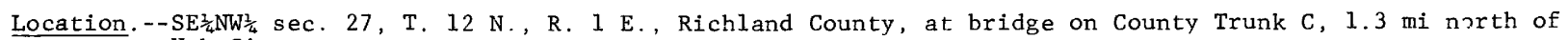
Hub City.

Drainage area. $-14.3 \mathrm{mi}^{2}$. Tributary to.--Pine River.

Type of site.--Miscellaneous site.

Discharge measurements.--Oct. $18,1972,8.90 \mathrm{ft}^{3} / \mathrm{s} ;$ June $3,1974,13.2 \mathrm{ft}^{3} / \mathrm{s}$.

05406718 Pine River near Hub City, Wis.

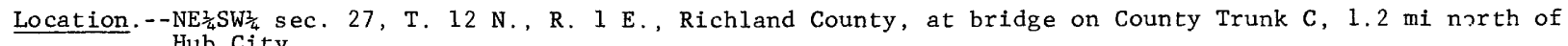
Hub City.

Drainage area. $--55.5 \mathrm{mi}^{2}$.

Tributary to. --Wisconsin River.

Type of site.--Miscellaneous site.

Discharge measurement. - - Oct. $18,1972,39.55 \mathrm{ft}^{3} / \mathrm{s}$.

05406719 Soules Creek at Hub City, Wis.

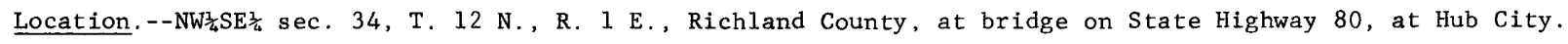
Drainage area. $--4.40 \mathrm{mi}^{2}$. Tributary to.--Pine River.

Type of site.--Miscellaneous site.

Discharge measurements.--Oct. $18,1972,1.86 \mathrm{ft}^{3} / \mathrm{s} ;$ June $3,1974,3.01 \mathrm{ft}^{3} / \mathrm{s}$.

05406722 Hawkins Creek at Hub City, Wis.

Location. - $-\mathrm{SW}_{4}^{\frac{1}{4}} \mathrm{NE} \frac{3}{4} \mathrm{sec} .3, \mathrm{~T} .11 \mathrm{~N} .$, R. $1 \mathrm{E}$. , Richland County, at bridge on private road $0.7 \mathrm{mi}$ south of Hub City.

Drainage area. $--15.2 \mathrm{mi}^{2}$.

Tributary to.--Pine River.

Type of site.--Miscellaneous site.

Discharge measurements.--Oct. $18,1972,10.2 \mathrm{ft}^{3} / \mathrm{s}$; June 3, 1974, $14.1 \mathrm{ft} / \mathrm{s}$. 
05406728 West Branch Pine River near Bloom City, Wis.

Location. - - NW $\frac{1}{4} \mathrm{NW} \frac{1}{4} \mathrm{sec} .15, \mathrm{~T}, 12 \mathrm{~N} ., \mathrm{R} .1 \mathrm{~W}$, Richland County, about $500 \mathrm{ft}$ below mouth of Basswood Creek, $2.2 \mathrm{mi}$ northwest of Bloom City.

Drainage area. $--10.5 \mathrm{mi}^{2}$. Tributary to. --Pine River.

Type of site.--Miscellaneous site.

Discharge measurements. --June $4,1974,6.89 \mathrm{ft}^{3} / \mathrm{s} ;$ Sept. 9, $1974,5.57 \mathrm{ft}^{3} / \mathrm{s} ;$ May 15, 1975, $5.87 \mathrm{ft} / \mathrm{s}$; June $17,1975,9.44 \mathrm{ft}^{3} / \mathrm{s}$.

Low-flow frequency. $-Q_{7,2}=3.2 \mathrm{ft}^{3} / \mathrm{s}, Q_{7,10}=2.5 \mathrm{ft}^{3} / \mathrm{s}$.

Basis of estimate. - - Correlated with Kickapoo River at La Farge using 4 discharge measurements.

Accuracy. $-\mathrm{SE}_{7,10}=19$ percent (average value for basin).

05406730 West Branch Pine River near Bloom City, Wis.

Location. - - $\mathrm{NE}_{\frac{1}{4} \mathrm{NE}} \frac{1}{4} \mathrm{sec} .22$, T. $12 \mathrm{~N} ., \mathrm{R} .1 \mathrm{~W} .$, Richland County, at bridge on country road, $0.2 \mathrm{mi}$ west of County Trunk Highway D, i.0 mi northwest of Bloom City.

Drainage area. $--13.0 \mathrm{mi}^{2}$.

Tributary to.--Pine River.

Type of site.--Miscellaneous site.

Discharge measurements.--June $4,1974,9.01 \mathrm{ft}^{3} / \mathrm{s}$; Sept. 9, 1974, $8.16 \mathrm{ft}^{3} / \mathrm{s} ;$ May $15,1975,7.75 \mathrm{ft}^{3} / \mathrm{s}$;

Low-flow frequency. $-Q_{7,2}=4.0 \mathrm{ft}^{3} / \mathrm{s}, Q_{7,10}=3.2 \mathrm{ft}^{3} / \mathrm{s}$.

Basis of estimate.--Correlated with Kickapoo River at La Farge using 4 discharge measurements.

Accuracy. $--\mathrm{SE}_{7,10}=19$ percent (average value for basin).

05406732 West Branch Pine River near Bloom City, Wis.

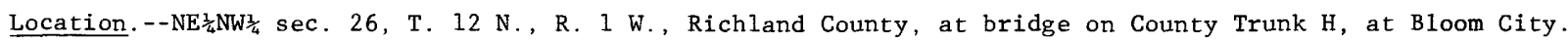
Drainage area. $--14.6 \mathrm{mi}^{2}$. Tributary to.--Pine River.

Type of site.--Miscellaneous site.

Discharge measurements.--Aug. $19,1970,5.85 \mathrm{ft}^{3} / \mathrm{s} ;$ Oct. $17,1972,8.89 \mathrm{ft}^{3} / \mathrm{s} ;$ June $4,1974,10.8 \mathrm{ft}^{3} / \mathrm{s}$. Low-flow frequency. $-Q_{7,2}=4.3 \mathrm{ft}^{3} / \mathrm{s}, 0.7,10=3.4 \mathrm{ft}^{3} / \mathrm{s}$.

Basis of estimate. - - Correlated with Kickapoo River at La Farge using 3 discharge measurements.

Accuracy. $-\mathrm{SE}_{7,10}=19$ percent (average value for basin).

05406735 West Branch Pine River tributary near Bloom City, Wis.

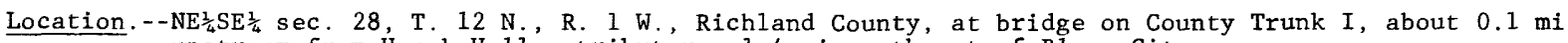
upstream from Hynek Hollow tributary, $1.4 \mathrm{mi}$ southwest of Bloom City.

Drainage area. $--2.47 \mathrm{mi}^{2}$.

Tributary to. --West Branch Pine River.

Type of site.--Miscellaneous site.

Discharge measurements.--June $4,1974,2.33 \mathrm{ft}^{3} / \mathrm{s}$; Sept. 10, $1974,2.48 \mathrm{ft}{ }^{3} / \mathrm{s}$; May $14,1975,2.33 \mathrm{ft}^{3} / \mathrm{s}$.

05406737 Tributary to West Branch Pine River tributary near Bloom City, Wis.

Location.-- $-\mathrm{SE}_{\frac{1}{4}} \mathrm{SE} \frac{1}{4} \mathrm{sec} .28, \mathrm{~T} .12 \mathrm{~N}, \mathrm{R} .1 \mathrm{~W}$, Richland County, at bridge on country road, $0.2 \mathrm{mi}$ south of County Trunk I, 0.5 mi upstream from confluence with Gault Hollow, 1.7 mi southwest of Bloon City.

Drainage area. $--2.59 \mathrm{mi}^{2}$.

Tributary to. --West Branch Pine River tributary.

Type of site.--Miscellaneous site.

Discharge measurements.--June $4,1974,1.57 \mathrm{ft}^{3} / \mathrm{s} ;$ Sept. $10,1974,1.42 \mathrm{ft}^{3} / \mathrm{s} ;$ June $16,1975,2.16 \mathrm{ft}^{3} / \mathrm{s}$.

Low-flow frequency. $-Q_{7,2}=0.74 \mathrm{ft}^{3} / \mathrm{s}, Q_{7,10}=0.60 \mathrm{ft}^{3} / \mathrm{s}$.

Basis of estimate.--Correlated with Kickapoo River at La Farge using 3 discharge measurements made during the period $1974-75$.

Accuracy.-- $-\mathrm{SE}_{7,10}=19$ percent (average value for basin). 
05406740 West Branch Pine River tributary at Bloom City, Wis.

Location.
Bloom City. Drainage area. $-6.13 \mathrm{mi}^{2}$. Tributary to.--West Branch Pine River.

Type of site.--Miscellaneous site.

Discharge measurements. - - Oct. $17,1972,4.16 \mathrm{ft}^{3} / \mathrm{s} ;$ June 4, 1974, 5.29 $\mathrm{ft}^{3} / \mathrm{s} ; \mathrm{Sept} .10,1974,4.40 \mathrm{ft} / \mathrm{s}$; Low-flow frequency. $-Q_{7,2}=2.4 \mathrm{ft}^{3} / \mathrm{s}, Q_{7,10}=1.8 \mathrm{ft}^{3} / \mathrm{s}$. Basis of estimate.-- Correlated with Kickapoo River at La Farge using 4 discharge measurements made during the
period 1972-75.

Accuracy. $--\mathrm{SE}_{7,10}=19$ percent (average value for basin).

05406743 West Branch Pine River near Rockbridge, Wis.

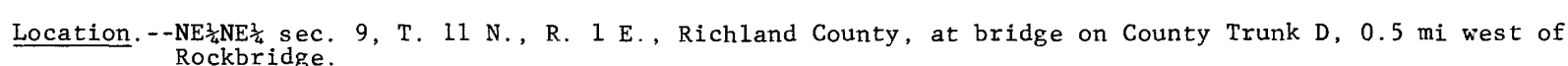
Drainage area. $--37.6 \mathrm{mi}^{2}$. Tributary to.--Pine River.

Type of site.--Miscellaneous site.

Discharge measurements.--Aug. 19, 1970, $16.4 \mathrm{ft}^{3} / \mathrm{s} ;$ Oct. 18, 1972, $20.3 \mathrm{ft}^{3} / \mathrm{s} ; \mathrm{June} 3,1974,30.7 \mathrm{ft} / \mathrm{s}$. Low-flow frequency. $-\mathrm{Q}_{7,2}=12 \mathrm{ft}^{3} / \mathrm{s} ; \mathrm{Q}_{7,10}=9.4 \mathrm{ft}^{3} / \mathrm{s}$.

Basis of estimate.--Correlated with Kickapoo River at La Farge using 3 discharge measurements made during the period 1970-74.

Accuracy. $--\mathrm{SE}_{7,10}=19$ percent (average value for basin).

05406745 Pine River at Rockbridge, Wis.

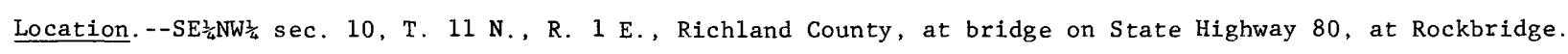
Drainage area. $--117 \mathrm{mi}^{2}$. Tributary to.--Wisconsin River.

Type of site.--Miscellaneous site.

Discharge measurements.--Aug. 19, 1970, $48.1 \mathrm{ft}^{3} / \mathrm{s} ;$ Oct. 18, 1972, 76.2 ft $/ \mathrm{s} ; \mathrm{June} 3,1974,102 \mathrm{ft} / \mathrm{s}$. Low-flow frequency. $-Q_{7,2}=38 \mathrm{ft}^{3} / \mathrm{s}, Q_{7,10}=29 \mathrm{ft}^{3} / \mathrm{s}$.

Basis of estimate.--Correlated with Kickapoo River at La Farge using 3 discharge measurements made during Accuracy. $-\mathrm{SE}_{7,10}=19$ percent (average value for basin).

05406748 South Buck Creek at Buck Creek, Wis.

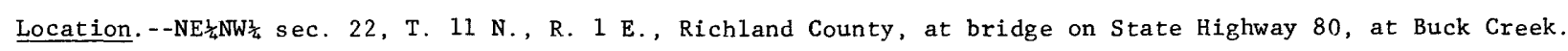
Drainage area. $--4.95 \mathrm{mi}^{2}$. Tributary to.--Pine River.

Type of site.--Miscellaneous site.

Discharge measurements.--0ct. 18, 1972, $1.25 \mathrm{ft}^{3} / \mathrm{s} ;$ June 3, $1974,2.08 \mathrm{ft}^{3} / \mathrm{s}$. 
05406750 Pine River near Buck Creek, Wis.

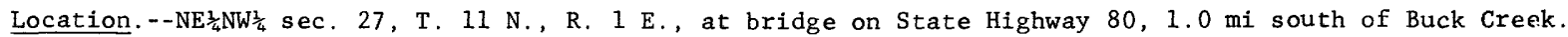
Drainage area. $--128 \mathrm{mi}^{2}$. Tributary to. --Wisconsin River.

Type of site.--Low-flow partial-record station.

Minimum discharge measured. $--48.8 \mathrm{ft}^{3} / \mathrm{s}$, Sept. 7, 1967.

Low-flow frequency. $-Q_{7,2}=46 \mathrm{ft}^{3} / \mathrm{s}, Q_{7,10}=37 \mathrm{ft}^{3} / \mathrm{s}$.

Basis of estimate. - Correlated with Kickapoo River at La Farge using 10 discharge measurements made ir the period 1963-76.

Accuracy. - $-\mathrm{SE}_{7,10}=10$ percent.

05406752 Fancy Creek near Bloom City, Wis.

Location.-- $\mathrm{NE}_{\frac{1}{4}} \mathrm{SW}_{\frac{1}{4}} \mathrm{sec} .4, \mathrm{~T} .11 \mathrm{~N} ., \mathrm{R} .1 \mathrm{~W}$, Richland County, at bridge on State Highway $56,3.2 \mathrm{mi}$ southwest of Bloom City.

Drainage area. $--4.14 \mathrm{mi}^{2}$. Tributary to. --Pine River.

Type of site.--Miscellaneous site.

Discharge measurements.--June $3,1974,1.94 \mathrm{ft}^{3} / \mathrm{s}$; Sept. 9, 1974, $1.62 \mathrm{ft} \mathrm{t}^{3} / \mathrm{s}$; May 15, 1975, $2.01 \mathrm{ft} / \mathrm{s}$. Low-flow frequency. $-Q_{7,2}=1.2 \mathrm{ft}^{3} / \mathrm{s}, Q_{7,10}=1.0 \mathrm{ft}^{3} / \mathrm{s}$.

Basis of estimate.--Correlated with Kickapoo River at La Farge using 3 discharge measurements made during the period 1974-75.

Accuracy.-- $\mathrm{SE}_{7,10}=19$ percent (average value for the basin).

05406754 Fancy Creek near Gillingham, Wis.

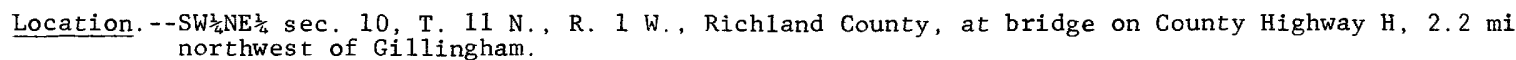

Drainage area. $--6.67 \mathrm{mi}^{2}$. Tributary to. --Pine River.

Type of site.--Miscellaneous site.

Discharge measurements.--June 3, 1974, $4.11 \mathrm{ft} / 3$; Sept. 9, 1974, $3.48 \mathrm{ft}^{3} / \mathrm{s}$; May 15, $1975,4.66 \mathrm{ft} / \mathrm{s}$. Low-flow frequency. $-Q_{7,2}=2.5 \mathrm{ft}^{3} / \mathrm{s}, Q_{7,10}=2.1 \mathrm{ft}^{3} / \mathrm{s}$.

Basis of estimate.--Correlated with Kickapoo River at La Farge using 3 discharge measurements made during the period $1974-75$.

Accuracy. $--\mathrm{SE}_{7,10}=19$ percent (average value for the basin).

05406756 Fancy Creek near Gillingham, Wis .

Location. - - $-\mathrm{SW}_{\frac{1}{4}} \mathrm{NE}^{\frac{1}{4}} \mathrm{sec} .14, \mathrm{~T} .11 \mathrm{~N} ., \mathrm{R} .1 \mathrm{~W} .$, Richland County, at bridge on County Trunk $Z$, near Gillingham. Drainage area. $--8.60 \mathrm{mi}^{2}$. Tributary to.--Pine River.

Type of site.--Miscellaneous site.

Discharge measurements. --0ct. 17, 1972, $4.32 \mathrm{ft}^{3} / \mathrm{s} ;$ June 9, 1974, $5.65 \mathrm{ft}^{3} / \mathrm{s}$.

05406757 Fancy Creek tributary near Gillingham, Wis.

Location.-- NW $\frac{1}{4} \mathrm{NE}_{\frac{1}{4}} \mathrm{sec} .16, \mathrm{~T} .11 \mathrm{~N} ., \mathrm{R} .1 \mathrm{~W}$. , Richland County, at bridge on private drive, $2.7 \mathrm{mi}$ northwest of Gillingham.

Drainage area. $--1.69 \mathrm{mi}^{2}$.

Tributary to. --Fancy Creek.

Type of site.--Miscellaneous site.

Discharge measurements. - June 3, 1974, $0.734 \mathrm{ft}_{3}^{3} / \mathrm{s}$; Sept. 9, $1974,0.71 \mathrm{ft}{ }^{3} / \mathrm{s} ;$ May $15,1975,0.79 \mathrm{ft}^{3} / \mathrm{s}$;

Low-flow frequency. $-Q_{7,2}=0.35 \mathrm{ft}^{3} / \mathrm{s}, Q_{7,10}=0.28 \mathrm{ft}^{3} / \mathrm{s}$.

Basis of estimate.--Correlated with Kickapoo River at La Farge using 4 discharge measurements made during the period 1974-75.

Accuracy. - $-\mathrm{SE}_{7,10}=19$ percent (average value for the basin). 
05406758 Fancy Creek tributary near Gil1ingham, Wis.

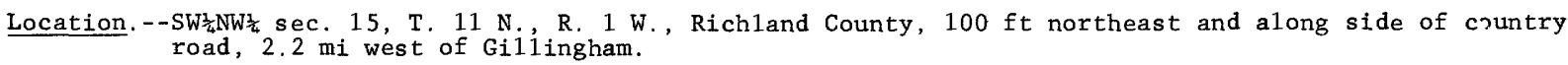

Drainage area. $--2.20 \mathrm{mi}^{2}$. Tributary to.--Fancy Creek.

Type of site.--Miscellaneous site.

Discharge measurements.--June $3,1974,1.64 \mathrm{ft}^{3} / \mathrm{s} ;$ Sept. 9, 1974, $0.790 \mathrm{ft}^{3} / \mathrm{s} ; \mathrm{May} 15,1975,0.95 \mathrm{ft} / \mathrm{s}$; June $16,1975,1.31 \mathrm{ft}^{3} / \mathrm{s}$.

Low-flow frequency. $-Q_{7,2}=0.41 \mathrm{ft}^{3} / \mathrm{s}, Q_{7,10}=0.30 \mathrm{ft}^{3} / \mathrm{s}$.

Basis of estimate.--Correlated with Kickapoo River at La Farge using 4 discharge measurements made during the period 1974-75.

Accuracy. $--\mathrm{SE}_{7,10}=19$ percent (average value for basin).

05406763 Tributary to Fancy Creek tributary near Gillingham, Wis.

Location. - - SW $\frac{1}{4} N W \frac{1}{4}$ sec. 22, T. 11 N., R. 1 W. Richland County, 200 ft below confluence of unnamed tributaries, 2.1 mi southwest of Gillingham.

Drainage area. $-2.13 \mathrm{mi}^{2}$. Tributary to.--Fancy Creek tributary.

Type of site.--Miscellaneous site.

Discharge measurements.--June 3, 1974, $1.45 \mathrm{ft}^{3} / \mathrm{s} ;$ Sept. 10, 1974, $1.17 \mathrm{ft}^{3} / \mathrm{s} ; \mathrm{May} 14,1975,1.49 \mathrm{ft}^{3}$; s. Low-flow frequency. $-\mathrm{Q}_{7,2}=0.84 \mathrm{ft}^{3} / \mathrm{s}, \mathrm{Q}_{7,10}=0.74 \mathrm{ft}^{3} / \mathrm{s}$.

Basis of estimate.--Correlated with Kickapoo River at La Farge using 3 discharge measurements.

Accuracy. $--\mathrm{SE}_{7,10}=19$ percent (average value for basin).

05406764 Tributary to Fancy Creek tributary near Gillingham, Wis.

Location. - $-\mathrm{SE}_{\frac{1}{4}} \mathrm{SE}_{\frac{1}{4}}$ sec. $15, \mathrm{~T} .11 \mathrm{~N} ., \mathrm{R} .1 \mathrm{~W} .$, Richland County, at bridge on County Trunk $\mathrm{z}$, 1.3 mi west of Gillingham .

Drainage area. $--2.73 \mathrm{mi}^{2}$. Tributary to.--Fancy Creek tributary.

Type of site.--Miscellaneous site.

Discharge measurements.--June 3, 1974, $1.83 \mathrm{ft}^{3} / \mathrm{s}$; Sept. 10, 1974, $1.53 \mathrm{ft}^{3} / \mathrm{s} ; \mathrm{May} 14,1975,1.82 \mathrm{ft} / \mathrm{s}$. Low-flow frequency. $-Q_{7,2}=1.2 \mathrm{ft}^{3} / \mathrm{s}, Q_{7,10}=1.1 \mathrm{ft}^{3} / \mathrm{s}$. Basis of estimate.--Correlated with Kickapoo River at La Farge using 3 discharge measurements made during
the period 1974-75.

Accuracy. $--\mathrm{SE}_{7,10}=19$ percent (average value for basin).

05406765 Fancy Creek tributary near Gillingham, Wis.

Location.-- SW $\frac{1}{4}$ SW $\frac{1}{4}$ sec. 14, T. 11 N., R. 1 W., Richland County, at bridge on private road, 1.0 mi west of
Gillingham.

Drainage area. $--6.21 \mathrm{mi}^{2}$.

Tributary to. --Fancy Creek.

Type of site.--Miscellaneous site.

Discharge measurements. --oct. $17,1972,3.70 \mathrm{ft}^{3} / \mathrm{s} ;$ June $4,1974,3.65 \mathrm{ft}^{3} / \mathrm{s}$.

05406770 Fancy Creek tributary at Gillingham, Wis.

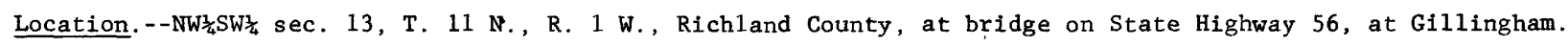

Drainage area. $--2.28 \mathrm{mi}^{2}$.

Tributary to.--Fancy Creek.

Type of site.--Miscellaneous site.

Discharge measurements.--oct. $17,1972,1.04 \mathrm{ft}^{3} / \mathrm{s} ;$ June 4, 1974, $1.62 \mathrm{ft}^{3} / \mathrm{s}$ 
Table 1.--Low-flow characteristics for sites in the lower Wisconsin River basin--Continued

05406780 Fancy Creek near Richland Center, Wis.

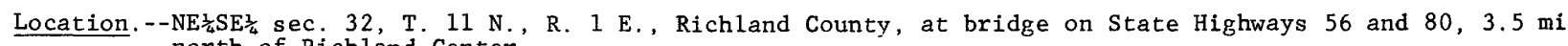
north of Richland Center.

Drainage area. $--28.4 \mathrm{mi}^{2}$. Tributary to.--Pine River.

Type of site.--Miscellaneous site.

Discharge measurements.--Aug. 19, 1970, $10.7 \mathrm{ft}^{3} / \mathrm{s} ;$ Oct. $17,1972,13.4 \mathrm{ft}^{3} / \mathrm{s} ;$ June $3,1974,20.2 \mathrm{ft}^{3} / \mathrm{s}$. Low-flow frequency. $-Q_{7,2}=8.8 \mathrm{ft}^{3} / \mathrm{s}, Q_{7,10}=7.6 \mathrm{ft}^{3} / \mathrm{s}$.

Basis of estimate.--Correlated with Kickapoo River at La Farge using 3 discharge measurements made during the period 1970-74.

Accuracy. $-\mathrm{SE}_{7,10}=19$ percent (average value for basin).

\section{Horse Creek near Gillingham, Wis.}

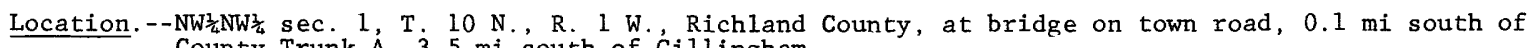
County Trunk A, $3.5 \mathrm{mi}$ south of Gillingham.

Drainage area. $--3.44 \mathrm{mi}^{2}$. Tributary to.--Pine River.

Type of site.--Miscellaneous site.

Discharge measurements.--June 3, 1974, $2.50 \mathrm{ft}^{3} / \mathrm{s}$; Sept. $10,1974,1.81 \mathrm{ft} / \mathrm{s}$; May 14, 1975, $2.50 \mathrm{ft} / \mathrm{s}$. Low-flow frequency. $-Q_{7,2}=1.1 \mathrm{ft}^{3} / \mathrm{s}, Q_{7,10}=0.93 \mathrm{ft}^{3} / \mathrm{s}$.

Basis of estimate.-- Correlated with Kickapoo River at La Farge using 3 discharge measurements made during the period 1974-75.

Accuracy. - $-\mathrm{SE}_{7,10}=19$ percent (average value for basin).

05406782 Horse Creek tributary near Gillingham, Wis.

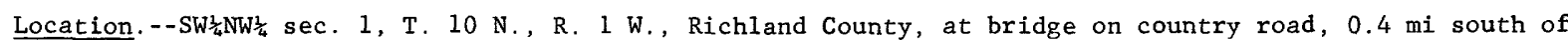
County Trunk A, $3.7 \mathrm{mi}$ south of Gillingham.

Drainage area. $--1.47 \mathrm{mi}^{2}$. Tributary to. --Horse Creek.

Type of site.--Miscellaneous site.

Discharge measurements.--June 3, 1974, $1.02 \mathrm{ft}^{3} / \mathrm{s}$; Sept. 10, 1974, $0.50 \mathrm{ft} / \mathrm{s} ;$ May 14, $1975,0.86 \mathrm{ft}^{3} / \mathrm{s}$. Low-flow frequency. $-Q_{7,2}=0.22 \mathrm{ft}^{3} / \mathrm{s}, Q_{7,10}=0.15 \mathrm{ft}^{3} / \mathrm{s}$.

Basis of estimate.--Correlated with Kickapoo River at La Farge based on 3 discharge measurements made during the period 1974-75.

Accuracy.-- $\mathrm{SE}_{7,10}=19$ percent (average value for basin).

05406783 Horse Creek near Richland Center, Wis.

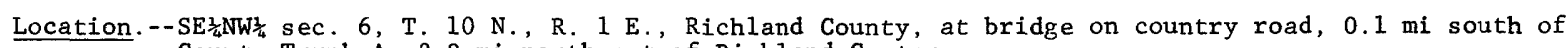
County Trunk A, $2.9 \mathrm{mi}$ northwest of Richland Center.

Drainage area. $--7.42 \mathrm{mi}^{2}$

Tributary to.--Pine River.

Type of site.--Miscellaneous site.

Discharge measurements.--June 3, 1974, $4.58 \mathrm{ft}^{3} / \mathrm{s}$; Sept. 10, 1974, $3.43 \mathrm{ft} / \mathrm{s}$; May 14, $1975,4.76 \mathrm{ft} / 3$. Low-flow frequency. $-Q_{7,2}=2.2 \mathrm{ft}^{3} / \mathrm{s}, Q_{7,10}=1.9 \mathrm{ft}^{3} / \mathrm{s}$.

Basis of estimate.--Correlated with Kickapoo River at La Farge using 3 discharge measurements made during the period 1974-75.

Accuracy. $-\mathrm{SE}_{7,10}=19$ percent (average value for basin). 
05406784 Horse Creek near Richland Center, Wis.

Location. - - SW $\frac{1}{6} \mathrm{SE} \frac{1}{4} \mathrm{sec} .5, \mathrm{~T} .10 \mathrm{~N} .$, R. 1 E., Richland County, at bridge on State Highway 56-80, 2.2 mi north of Richland Center.

Drainage area. $--8.90 \mathrm{mi}^{2}$. Tributary to. - -Pine River.

Type of site.--Miscellaneous site.

Discharge measurements. --Oct. $18,1972,4.31 \mathrm{ft}^{3} / \mathrm{s} ;$ June $3,1974,5.70 \mathrm{ft}^{3} / \mathrm{s}$.

05406785 Brush Creek near Richland Center, Wis.

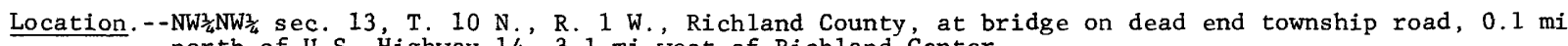
north of U.S. Highway $14,3.1 \mathrm{mi}$ west of Richland Center.

Drainage area. $-1.29 \mathrm{mi}^{2}$.

Tributary to. - Pine River.

Type of site.--Miscellaneous site.

Discharge measurements.--June 3, 1974, $0.85 \mathrm{ft}^{3} / \mathrm{s}$; Sept. 9, $1974,0.422 \mathrm{ft}^{3} / \mathrm{s}$; May $14,1975,0.84 \mathrm{ft}^{3} / \mathrm{s}$. Low-flow frequency. $-Q_{7,2}=0.15 \mathrm{ft}^{3} / \mathrm{s}, Q_{7,10}=0.10 \mathrm{ft}^{3} / \mathrm{s}$.

Basis of estimate. --Correlated with Kickapoo River at La Farge using 3 discharge measurements.

Accuracy. $-\mathrm{SE}_{7,10}=19$ percent (average value for basin).

05406786 Brush Creek tributary near Richland Center, Wis.

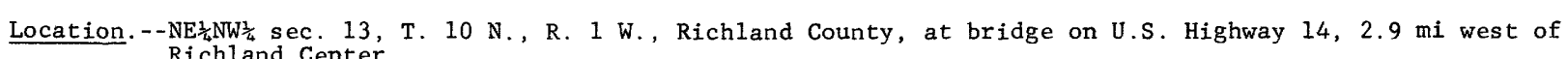
Drainage area. $-1.32 \mathrm{mi}^{2}$

Type of site.--Miscellaneous site.

Discharge measurements. --June 3, 1974, $1.08 \mathrm{ft}^{3} / \mathrm{s}$; Sept. 9, 1974, $0.40 \mathrm{ft}^{3} / \mathrm{s}$; May $14,1975,1.11 \mathrm{ft} / \mathrm{s}$; June $16,1975,1.42 \mathrm{ft}^{3} / \mathrm{s}$.

Low-flow frequency. - $-Q_{7,2}=0.09 \mathrm{ft}^{3} / \mathrm{s}, Q_{7,10}=0.04 \mathrm{ft}^{3} / \mathrm{s}$.

Basis of estimate.--Correlated with Kickapoo River at La Farge based on 4 discharge measurements.

Accuracy. $-\mathrm{SE}_{7,10}=19$ percent (average value for basin).

05406787 Brush Creek near Richland Center, Wis.

Location. - - NE $\frac{1}{4} \mathrm{NE}_{\frac{1}{4}}$ sec. 13, T. $10 \mathrm{~N} .$, R. 1 W., Richland County, at bridge on private road, $2.5 \mathrm{mi}$ west of Richland Center.

Drainage area. $--3.13 \mathrm{mi}^{2}$.

Tributary to.--Pine River.

Type of site.--Miscellaneous site.

Discharge measurements.--June $3,1974,2.55 \mathrm{ft}^{3} / \mathrm{s} ;$ Sept. $9,1974,1.94 \mathrm{ft}^{3} / \mathrm{s} ;$ May $14,1975,2.14 \mathrm{ft}^{3} / \mathrm{s}$;

Low-flow frequency. $-Q_{7,2}=1.5 \mathrm{ft}^{3} / \mathrm{s}, Q_{7,10}=1.3 \mathrm{ft}^{3} / \mathrm{s}$.

Basis of estimate.--Correlated with Kickapoo River at La Farge using 4 discharge measurements.

Accuracy. - $-\mathrm{SE}_{7,10}=19$ percent (average value for basin).

05406789 Brush Creek at Richland Center, Wis.

Location. -- $\mathrm{SW}_{\frac{1}{4}} \mathrm{NE}_{\frac{1}{4}} \mathrm{sec} .17, \mathrm{~T} .10 \mathrm{~N} ., \mathrm{R} .1 \mathrm{E} .$, Richland County, at bridge on private road, in Richland Center. Drainage area. $--6.77 \mathrm{mi}^{2}$.

Tributary to.--Pine River.

Type of site.--Miscellaneous site.

Discharge measurements.--Oct. 18, 1972, $3.07 \mathrm{ft}^{3} / \mathrm{s} ;$ June $3,1974,4.03 \mathrm{ft} / \mathrm{s}$. 
05406793 Center Creek at Richland Center, Wis.

Location.--SW $\frac{1}{4} \mathrm{NE}_{\frac{1}{4}} \mathrm{sec} .20$, T. $10 \mathrm{~N} .$, R. I E., Richland County, at bridge on city street, in Richland Center. Drainage area. $--2.57 \mathrm{mi}^{2}$. Tributary to.--Pine River.

Type of site.--Miscellaneous site.

Discharge measurements. --Oct. 18, 1972, $0.872 \mathrm{ft}^{3} / \mathrm{s} ;$ June $3,1974,1.32 \mathrm{ft}^{3} / \mathrm{s}$.

05406795 Pine River at Richland Center, Wis.

Location.--SW3 $\frac{1}{4} \mathrm{NW}_{\frac{1}{4}} \mathrm{sec} .21, \mathrm{~T} .10 \mathrm{~N} ., \mathrm{R} .1 \mathrm{E}$. , Richland County, at sewage-treatment plant, at Richland Center. Drainage area. $--190 \mathrm{mi}^{2}$. Tributary to. --Wisconsin River.

Type of site.--Miscellaneous site.

Discharge measurements. --Oct. $18,1972,110 \mathrm{ft}^{3} / \mathrm{s} ;$ June $27,1973,189 \mathrm{ft}_{3}^{3} / \mathrm{s} ;$ Aug. $22,1973,129 \mathrm{ft} / \mathrm{s} ;$ Low-flow frequency. $-Q_{7,2}=65 \mathrm{ft}^{3} / \mathrm{s}, Q_{7,10}=55 \mathrm{ft} / \mathrm{s}$.

Basis of estimate.--Correlated with Baraboo River near Baraboo using 5 discharge measurements. Accuracy. $--\mathrm{SE}_{7,10}=10$ percent.

05406800 Rocky Branch near Richland Center, Wis.

Location. - - $\mathrm{NE}_{\frac{2}{4}} \mathrm{SE} \frac{1}{4} \mathrm{sec} .29$, T. $10 \mathrm{~N}$, R. $1 \mathrm{E}$, Richland County, at culvert on State Highway $80,1.5 \mathrm{mi}$ south of Richland Center.

Drainage area. $--1.68 \mathrm{mi}^{2}$. Tributary to.--Pine River.

Type of site.--Miscellaneous site.

Discharge measurements.--Oct. $18,1972,0.586 \mathrm{ft}^{3} / \mathrm{s} ;$ June $3,1974,0.64 \mathrm{ft}^{3} / \mathrm{s}$.

05406810 Pine River at Richland Center, Wis.

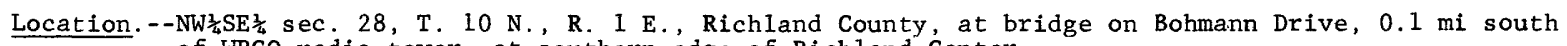
of WRCO radio tower, at southern edge of Richland Center.

Drainage area. $--194 \mathrm{mi}^{2}$.

Tributary to. --Wisconsin River.

Type of site.--Miscellaneous site.

Discharge measurements.--June 4,1974,152 $\mathrm{ft}^{3} / \mathrm{s}$; Sept. 9, 1974, $126 \mathrm{ft}^{3} / \mathrm{s} ;$ May 15, 1975, $163 \mathrm{ft}^{3} / \mathrm{s}$; June $17,1975,210 \mathrm{ft} 3 / \mathrm{s}$.

05406820 Pine River near Richland Center, Wis .

Location.-- $-\mathrm{SE}_{\frac{3}{4} \mathrm{SE}} \frac{1}{4} \mathrm{sec} .27, \mathrm{~T}, 10 \mathrm{~N} ., \mathrm{R} .1 \mathrm{E} .$, Richland County, at bridge on County Trunk $0,2.5 \mathrm{mi}$ southeast Drainage area. $--198 \mathrm{mi}^{2}$. Tributary to. --Wiscons in River.

Type of site.--Low-flow partial-record station.

Minimum discharge measured. $--62.3 \mathrm{ft}^{3} / \mathrm{s}$, July $30,1965$.

Low-flow frequency. $-Q_{7,2}=74 \mathrm{ft}^{3} / \mathrm{s}, Q_{7,10}=63 \mathrm{ft}^{3} / \mathrm{s}$. Basis of estimate.--Correlated with Kickapoo River at La Farge using 15 discharge measurements made in the
period $1963-76$.

Accuracy. $--\mathrm{SE}_{7,10}=10$ percent. 
05406825 Spring creek near Richland Center, Wis.

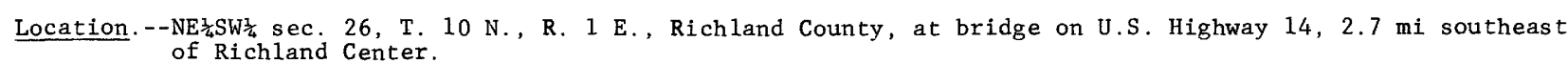
Drainage area. $--2.74 \mathrm{mi}^{2}$. Tributary to.--Pine River.

Type of site.--Miscellaneous site.

Discharge measurements.--0ct. 18, 1972, $0.56 \mathrm{ft}^{3} / \mathrm{s} ;$ June 4, 1974, $0.81 \mathrm{ft} / \mathrm{s}$.

05406832 Pine River near Twin Bluffs, Wis.

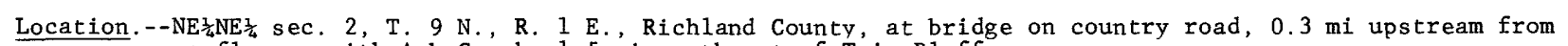
confluence with Ash Creek, $1.5 \mathrm{mi}$ northwest of Twin Bluffs.

Drainage area. $-204 \mathrm{mi}^{2}$.

Tributary to.--Wisconsin River.

Type of site.--Miscellaneous site.

Discharge measurement.--June $17,1975,260 \mathrm{ft}^{3} / \mathrm{s}$.

05406835 Ash Creek near Richland Center, Wis.

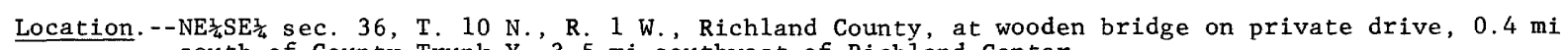
south of County Trunk Y, $3.5 \mathrm{mi}$ southwest of Richland Center.

Drainage area. $--3.00 \mathrm{mi}^{2}$.

Tributary to.--Pine River.

Type of site.--Miscellaneous site.

Discharge measurements. --June 3, 1974, $1.25 \mathrm{ft}^{3} / \mathrm{s} ;$ Sept. $10,1974,1.36 \mathrm{ft} / \mathrm{s} ; \mathrm{May} 14,1975,1.13 \mathrm{ft} / \mathrm{s}$.

05406840 Ash Creek near Richland Center, Wis.

Location.--
of Richlat $\frac{1}{4}$ sec. 6 , T. 9 N., R. 1 E., Richland County, at bridge on U.S. Highway $80,3.6$ mi so'tthwest

Drainage area. $--4.04 \mathrm{mi}^{2}$.

Tributary to.--Pine River.

Type of site.--Miscellaneous site.

Discharge measurements.--June $3,1974,2.15 \mathrm{ft}^{3} / \mathrm{s} ;$ Sept. $10,1974,2.18 \mathrm{ft} / \mathrm{s} ; \mathrm{May} 14,1975,1.97 \mathrm{ft} / \mathrm{s}$.

05406845 Ash Creek near Richland Center, Wis.

Location.--NW/ $\frac{1}{4} \frac{1}{4}$ sec. 9 , T. 9 N., R. 1 E., Richland County, at bridge on County Trunk 0 , 4.3 mi south of Richland Center.

Drainage area. $--7.95 \mathrm{mi}^{2}$. Tributary to.--Pine River.

Type of site.--Miscellaneous site.

Discharge measurements.--oct. $17,1972,3.46 \mathrm{ft}^{3} / \mathrm{s} ;$ June $4,1974,4.70 \mathrm{ft}^{3} / \mathrm{s}$.

05406850 Ash Creek near Richland Center, Wis.

Location.--SE $\frac{1}{4} N E \frac{1}{4}$ sec. 2, T. 9 N., R. 1 E., Richland County, at bridge on County Trunk TB, 4.0 mi so'theast of Richland Center.

Drainage area. $--18.4 \mathrm{mi}^{2}$.

Tributary to.--Pine River.

Type of site.--Low-flow partial-record station.

Minimum discharge measured. $--3.15 \mathrm{ft}^{3} / \mathrm{s}$, Sept. 7, 1967.

Low-flow frequency. $-\mathrm{Q}_{7,2}=3.0 \mathrm{ft}^{3} / \mathrm{s}, Q_{7,10}=2.4 \mathrm{ft}^{3} / \mathrm{s}$.

Basis of estimate.--Correlated with Kickapoo River at La Farge using 12 discharge measurements made in the period $1966-76$.

Accuracy. $--\mathrm{SE}_{7,10}=9$ percent. 
Table 1.--Low-flow characteristics for sites in the lower Wisconsin River basin--Continued

05406851 Pine River at Twin Bluffs, Wis.

Location. - - NW $\frac{1}{4} \mathrm{NW} \frac{1}{4}$ sec. 7, T. 9 N., R. 2 E., Richland County, at bridge on town road, $0.4 \mathrm{mi}$ upstream from confluence with Willow Creek, 0.2 mi nor theast of Twin Bluffs.

Drainage area. $--224 \mathrm{mi}^{2}$.

Tributary to. --Wisconsin River.

Type of site.--Miscellaneous site.

Discharge measurements.--June 4, 1974, $171 \mathrm{ft}^{3} / \mathrm{s}$; Sept, 9, 1974, $133 \mathrm{ft}^{3} / \mathrm{s} ;$ May 14, 1975, $183 \mathrm{ft}^{3} / \mathrm{s}$.

Low-flow frequency. $--Q_{7,2}=91 \mathrm{ft}^{3} / \mathrm{s}, Q_{7,10}=77 \mathrm{ft}^{3} / \mathrm{s}$.

Basis of estimate.--Correlated with Kickapoo River at La Farge using 3 discharge measurements.

Accuracy.--SE $7,10=19$ percent (average value for basin).

05406853 Willow Creek near Loyd, Wis.

Location. - $-\mathrm{SW}_{\frac{1}{4}} \mathrm{SE}_{\frac{1}{4}} \mathrm{sec} .10, \mathrm{~T} .11 \mathrm{~N}$, R 2 E., Richland County, $0.3 \mathrm{mi}$ upstream from bridge on State Highway 58, $1.2 \mathrm{mi}$ north of Loyd.

Drainage area. $--9.28 \mathrm{mi}^{2}$.

Tributary to.--Pine River.

Type of site.--Miscellaneous site.

Discharge measurements.--May 7, 1974, $4.59 \mathrm{ft}^{3} / \mathrm{s}$; Aug. 9, 1976, 2.42 $\mathrm{ft}^{3} / \mathrm{s} ; 0 \mathrm{ct} .26,1976,2.30 \mathrm{ft} / \mathrm{s}$.

Low-flow frequency. $-Q_{7,2}=1.8 \mathrm{ft}^{3} / \mathrm{s}, Q_{7,10}=1.4 \mathrm{ft}^{3} / \mathrm{s}$.

Basis of estimate.--Correlated with Kickapoo River at La Farge using 3 discharge measurements.

Accuracy. $-\mathrm{SE}_{7,10}=19$ percent (average value for basin).

05406855 Willow Creek tributary near Loyd, Wis.

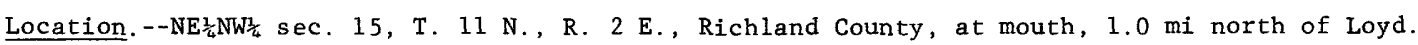

Drainage area. $--5.06 \mathrm{mi}^{2}$. Tributary to. - Willow Cre€k.

Type of site.--Miscellaneous site.

Discharge measurements.--May 7, 1974, $3.89 \mathrm{ft}^{3} / \mathrm{s} ;$ Aug, 9, 1976, $2.03 \mathrm{ft}^{3} / \mathrm{s} ;$ Oct. 26, $1976,1.99 \mathrm{ft} 3 / \mathrm{s}$.

05406858 Willow Creek tributary near Loyd, Wis.

Location. - -SW $\frac{1}{4} \mathrm{SW}_{\frac{1}{4}} \mathrm{sec} .14, \mathrm{~T} .11 \mathrm{~N} ., \mathrm{R} .2 \mathrm{E}$. , Richland County, $200 \mathrm{ft}$ above bridge on private road, $1.4 \mathrm{mi}$ east of Loyd.

Drainage area. $--3.67 \mathrm{mi}^{2}$.

Tributary to. --Willow Creek.

Type of site.--Miscellaneous site.

Discharge measurements. - May 7, 1974, $0.86 \mathrm{ft}^{3} / \mathrm{s}$; Aug. 9, 1976, $0.33 \mathrm{ft}^{3} / \mathrm{s} ; 0 \mathrm{ct} .26,1976,0.36 \mathrm{ft}^{3} / \mathrm{s}$.

Low-flow frequency. $-Q_{7,2}=0.24 \mathrm{ft}^{3} / \mathrm{s}, Q_{7,10}=0.15 \mathrm{ft}^{3} / \mathrm{s}$.

Basis of estimate.--Correlated with Kickapoo River at La Farge using 3 discharge measurements.

Accuracy.- $-\mathrm{SE}_{7,10}=19$ percent (average value for basin).

05406860 Tributary to Willow Creek tributary near Loyd, Wis.

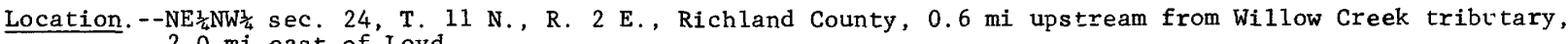
$2.0 \mathrm{mi}$ east of Loyd.

Drainage area. $--3.52 \mathrm{mi}^{2}$.

Tributary to.--Willow Creek tributary.

Type of site.--Miscellaneous site.

Discharge measurements.--May 7, 1974, $0.75 \mathrm{ft}^{3} / \mathrm{s}$; Aug. 9, 1976, $0.21 \mathrm{ft}^{3} / \mathrm{s} ; 0 \mathrm{ct} .26,1976,0.25 \mathrm{ft}^{3} / \mathrm{s}$.

Low-flow frequency. $-Q_{7,2}=0.14 \mathrm{ft}^{3} / \mathrm{s}, Q_{7,10}=0.09 \mathrm{ft}^{3} / \mathrm{s}$.

Basis of estimate. --Correlated with Kickapoo River at La Farge using 3 discharge measurements.

Accuracy. $--\mathrm{SE}_{7,10}=19$ percent (average value for basin). 
05406863 Willow Creek tributary near Loyd, Wis.

Location.-- $-\mathrm{SE}_{4} \frac{1}{4} \mathrm{SE} \frac{1}{4} \mathrm{sec} .22$, T. 11 N., R. 2 E., Richland County, at mouth, 0.9 mi southeast of Loyd. Drainage area. $--9.96 \mathrm{mi}^{2}$. Tributary to.--Willow Creek.

Type of site.--Miscellaneous site.

Discharge measurement.--May 7, 1974, $4.63 \mathrm{ft}^{3} / \mathrm{s}$.

05406864 Willow Creek tributary near Loyd, Wis.

Location. - -SE $\frac{1}{4} S W \frac{1}{4}$ sec. 26, T. 11 N., R. 2 E., Richland County, at bridge on farm drive, 1.0 mi abovi? mouth, $1.9 \mathrm{mi}$ southeast of Loyd.

Drainage area. $--2.63 \mathrm{mi}^{2}$. Tributary to.--Willow Creek.

Type of site.--Miscellaneous site.

Discharge measurement.--May $7,1974,1.22 \mathrm{ft}^{3} / \mathrm{s}$.

05406865 Willow Creek near Richland Center, Wis. Location. - - NE $\frac{1}{4} \mathrm{NW} \frac{1}{4}$ sec. 34, T. 11 N., R. 2 E., Richland County, at bridge on State Highway 58 , 7.6 m: northeast
of Richland Center.

Drainage area. $--32.4 \mathrm{mi}^{2}$. Tributary to. --Pine River

Type of site.--Miscellaneous site.

Discharge measurement.--Aug. 17, $1970,14.8 \mathrm{ft}^{3} / \mathrm{s}$.

05406867 Willow Creek tributary near Loyd, Wis.

Location. - $-\mathrm{NW} \frac{1}{4} \mathrm{NW} \frac{1}{4} \mathrm{sec} .34, \mathrm{~T} .11 \mathrm{~N}$, , R. 2 E., Richland County, at bridge on town road, 0.1 mi upstream from mouth, $2.1 \mathrm{mi}$ south of Loyd.

Drainage area. $--2.94 \mathrm{mi}^{2}$. Tributary to. - Willow Creek.

Type of site.--Miscellaneous site.

Discharge measurements. - May $7,1974,1.03 \mathrm{ft}^{3} / \mathrm{s} ;$ Aug. $9,1976,0.57 \mathrm{ft}^{3} / \mathrm{s} ; 0 \mathrm{ct} .26,1976,0.61 \mathrm{ft} / \mathrm{s}$.

05406875 Willow Creek near Richland Center, Wis.

Location. $--S W \frac{1}{4} N W \frac{1}{4}$ sec. 20, T. 10 N., R. 2 E., Richland County, at junction with Little Willow Creek, 5.0 mi east of Richland Center.

Drainage area. $--54.7 \mathrm{mi}^{2}$. Tributary to.--Pine River.

Type of site.--Miscellaneous site.

Discharge measurement.--May $7,1974,40.5 \mathrm{ft}^{3} / \mathrm{s}$.

05406877 Little Willow Creek near Neptune, Wis.

Location. - $\begin{gathered}-\mathrm{SW} \frac{1}{4} \mathrm{NW} \frac{1}{4} \mathrm{sec} . \\ \text { Neptune. }\end{gathered}$

Drainage area. $--6.71 \mathrm{mi}^{2}$. Tributary to.--Willow Creek.

Type of site.--Miscellaneous site.

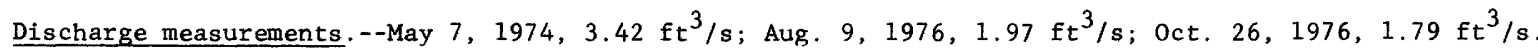
Low-flow frequency. $-\mathrm{Q}_{7,2}=1.4 \mathrm{ft}^{3} / \mathrm{s}, \mathrm{Q}_{7,10}=1.1 \mathrm{ft}^{3} / \mathrm{s}$.

Basis of estimate.--Correlated with Kickapoo River at La Farge using 3 discharge measurements. Accuracy. $-\mathrm{SE}_{7,10}=19$ percent (average value for basin). 
05406879 Little Willow Creek tributary near Ithaca, Wis.

Location. $--\mathrm{SE}_{\frac{1}{4}} \mathrm{SW} \frac{1}{4} \mathrm{sec} .7$, T. $10 \mathrm{~N}$, R. 2 E., Richland County, at mouth, $1.4 \mathrm{mi}$ northwest of Ithaca.

Drainage area. $--2.32 \mathrm{mi}^{2}$. Tributary to.--Little Willow Creek.

Type of site.--Miscellaneous site.

Discharge measurements.--May 7, 1974, $1.12 \mathrm{ft}^{3} / \mathrm{s}$; Aug. 9, 1976, $0.64 \mathrm{ft}^{3} / \mathrm{s} ; 0 \mathrm{ct} .26,1976,0.54 \mathrm{ft}^{3} / \mathrm{s}$. Low-flow frequency. $-Q_{7,2}=0.47 \mathrm{ft}^{3} / \mathrm{s}, Q_{7,10}=0.25 \mathrm{ft}^{3} / \mathrm{s}$.

Basis of estimate.--Correlated with Kickadoo River at La Farge using 3 discharge measurements.

Accuracy. $-\mathrm{SE}_{7,10}=19$ percent (average value for basin).

05406880 Little Willow Creek near Richland Center, Wis.

Location.-- $-\mathrm{SE}_{\frac{3}{4}} \mathrm{SW}_{\frac{1}{4}} \mathrm{sec} .7$, T. $10 \mathrm{~N} ., \mathrm{R} .2 \mathrm{E} .$, Richland County, at bridge on County Trunk N, $4.2 \mathrm{mi}$ east of Richland Center.

Drainage area. $--11.7 \mathrm{mi}^{2}$ Tributary to.--Willow Creek.

Type of site.--Miscellaneous site.

Discharge measurement. --Aug. 17, $1970,2.48 \mathrm{ft}^{3} / \mathrm{s}$.

05406882 Little Willow Creek near Richland Center, Wis.

Location. - - $-\mathrm{SW}_{4}^{\frac{1}{4}} \mathrm{NW}_{\frac{1}{4}} \mathrm{sec} .20$, T. $10 \mathrm{~N} .$, R. 2 E., Richland County, at mouth, 5.0 mi east of Richland Center. Drainage area. $--13.9 \mathrm{mi}^{2}$. Tributary to.--Willow Creek.

Type of site.--Miscellaneous site.

Discharge measurement.--May $7,1974,6.84 \mathrm{ft}^{3} / \mathrm{s}$

05406884 Hell Hollow near Aubrey, Wis.

Location.-- $\mathrm{NE}_{\frac{1}{4}} \mathrm{SE}_{\frac{1}{4}} \mathrm{sec} .30$, T. $10 \mathrm{~N} ., \mathrm{R} .2 \mathrm{E}$., Richland County, at County Trunk B bridge, 0.8 mi above mouth, $0.2 \mathrm{mi}$ s suth of Aubrey.

Drainage area. $--2.80 \mathrm{mi}^{2}$.

Tributary to.--Willow Creek.

Type of site.--Miscellaneous site.

Discharge measurement. --May 7, 1974, $0 \mathrm{ft}^{3} / \mathrm{s}$.

05406885 Willow Creek tributary near Sextonville, Wis.

Location. - - $\mathrm{NW}_{\frac{1}{4}} \mathrm{SE}_{\frac{1}{4}} \mathrm{sec} .31, \mathrm{~T} .10 \mathrm{~N}$, R. $2 \mathrm{E}$. , Richland County, at bridge on County Trunk B, $0.2 \mathrm{mi}$ upstream from mouth, $1.3 \mathrm{mi}$ north of Sextonville.

Drainage area. $--2.29 \mathrm{mi}^{2}$.

T.ibutary to.--Willow Creek.

Type of site.--Miscellaneous site.

Discharge measurement. --May $7,1974,0.46 \mathrm{ft}^{3} / \mathrm{s}$.

05406889 Willow Creek tributary at Sextonville, Wis.

Location.--SW $\frac{2}{4} \mathrm{SE}_{\frac{1}{4}} \mathrm{sec} .6$, T. 9 N., R. 2 E., Richland County, at bridge on County Trunk B, at Sextonville.

Drainage area. $--2.95 \mathrm{mi}^{2}$.

Tributary to.--Willow Creek.

Type of site.--Miscellaneous site.

Discharge measurements.--0ct. 7, 1972, $0.28 \mathrm{ft}^{3} / \mathrm{s} ;$ May 7, 1974, $0.36 \mathrm{ft}^{3} / \mathrm{s}$. 
05406890 Willow Creek near Richland Center, Wis.

Location. - -SE $\frac{1}{4}$ SW $\frac{1}{4}$ sec. 6, T. 9 N., R. 2 E., Richland County, 100 ft upstream from bridge on U.S. Highway 14, $6.0 \mathrm{mi}$ southeast of Richland Center.

Drainage area. $--82.5 \mathrm{mi}^{2}$. Tributary to.--Pine River.

Type of site.--Low-flow partial-record station.

Minimum discharge measured. $--26.5 \mathrm{ft}^{3} / \mathrm{s}, \mathrm{July} 30,1965$.

Low-flow frequency. $-Q_{7,2}=31 \mathrm{ft}^{3} / \mathrm{s}, Q_{7,10}=27 \mathrm{ft}^{3} / \mathrm{s}$.

Basis of estimate.--Correlated with Kickapoo River at La Farge using 16 discharge measurements made in the period $1963-76$.

Accuracy. $--\mathrm{SE}_{7,10}=8$ percent.

05406896 Pine River at Gotham, Wis.

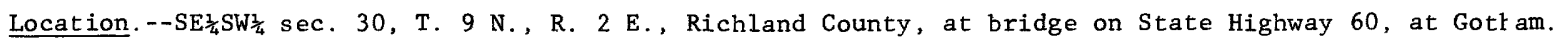
Drainage area. $--320 \mathrm{mi}^{2}$. Tributary to.--Wisconsin River.

Type of site.--Miscellaneous site.

Discharge measurements.--0ct. $17,1972,238 \mathrm{ft}^{3} / \mathrm{s} ;$ June $4,1974,286 \mathrm{ft}^{3} / \mathrm{s}$.

05407000 Wisconsin River at Muscoda, Wis.

Location. - $-\mathrm{SE} \frac{1}{4} \mathrm{NW} \frac{1}{4}$ sec. 1, T. $8 \mathrm{~N}$, , R. 1 W., Grant County, on left bank at bridge on State Highway 80 , $0.5 \mathrm{mi}$ upstream from Eagle Mili Creek, $1 \mathrm{mi}$ north of Muscoda.

Drainage area. $--10,450 \mathrm{mi}^{2}$.

Tributary to.--Mississippi River.

Type of site.--Gaging station.

Period of record.--October 1913 through September 1975.

Average discharge. -63 years, 8,625 $\mathrm{ft}^{3} / \mathrm{s}$.

Regulation.--Flow is regulated by 16 reservoirs before 1923, 17 reservoirs 1923-26, 18 reservoirs 1927-34, 20 reservoirs $1935-36,21$ reservoirs $1937-42,22$ reservoirs $1943-49$, 24 reservoirs since 1950 , and many powerplants upstream from this station.

Extremes.--Maximum discharge, $80,800 \mathrm{ft}^{3} / \mathrm{s}$ Sept. 16, 1938; minimum discharge, $2,000 \mathrm{ft}{ }^{3} / \mathrm{s}$ Feb. 11,1918 .

\begin{tabular}{|c|c|c|c|c|c|c|}
\hline \multirow[t]{2}{*}{$\begin{array}{l}\text { Period } \\
\text { of con- } \\
\text { secutive } \\
\text { days }\end{array}$} & \multicolumn{6}{|c|}{$\begin{array}{l}\text { Magnitude and frequency of annual low flow } \\
\text { Discharge, in cubic feet per second, for } \\
\text { indicated recurrence interval, in years }\end{array}$} \\
\hline & 2 & 5 & 10 & 20 & 50 & 100 \\
\hline $\begin{array}{r}7 \\
14 \\
30 \\
60 \\
90\end{array}$ & $\begin{array}{l}3,770 \\
3,920 \\
4,090 \\
4,360 \\
4,720\end{array}$ & $\begin{array}{l}3,100 \\
3,210 \\
3,360 \\
3,580 \\
3,830\end{array}$ & $\begin{array}{l}2,790 \\
2,890 \\
3,040 \\
3,250 \\
3,450\end{array}$ & $\begin{array}{l}2,560 \\
2,650 \\
2,810 \\
3,010 \\
3,170\end{array}$ & $\begin{array}{l}2,310 \\
2,400 \\
2,560 \\
2,770 \\
2,890\end{array}$ & $\begin{array}{l}2,160 \\
2,240 \\
2,420 \\
2,620 \\
2,720\end{array}$ \\
\hline
\end{tabular}

\begin{tabular}{|lccccc|}
\hline \multicolumn{5}{|c|}{ Duration table of daily flow } \\
\begin{tabular}{|c} 
Discharge, in cubic feet per second, which \\
was exceeded for indicated percent of tine
\end{tabular} \\
\hline $\begin{array}{c}\text { Percent } \\
\mathrm{ft}^{3} / \mathrm{s}\end{array}$ & 28,900 & 20,700 & 15,300 & 11,200 & 9,100 \\
\hline $\begin{array}{c}\text { Percent } \\
\mathrm{ft}^{3} / \mathrm{s}\end{array}$ & 70 & 50 & 60 & 70 & 80 \\
\hline $\begin{array}{c}\text { Percent } \\
\mathrm{ft}^{3} / \mathrm{s}\end{array}$ & 9,750 & 6,780 & 5,990 & 5,270 & 4,620 \\
\hline
\end{tabular}

Accuracy.--Not applicable due to large amount of regulation.

05407003 Mil1 Creek near Boaz, Wis.

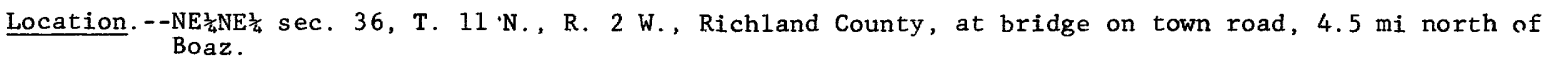

Drainage area. $--19.5 \mathrm{mi}^{2}$.

Tributary to. --Wisconsin River.

Type of site.--Miscellaneous site.

Discharge measurement.--Aug. 17, 1970, $6.17 \mathrm{ft}^{3} / \mathrm{s}$. 
05407013 Mi11 Creek near Boaz, Wis.

Location. - - - NW $\frac{1}{4} S W \frac{3}{4}$ sec. 32, T. 10 N., R. 1 W., Richland County, at bridge on County Trunk Q, 2.4 mi south of Drainage area. $--61.5 \mathrm{mi}^{2}$. Tributary to. --Wisconsin River.

Type of site.--Miscellaneous site.

Discharge measurement.--Aug, $19,1970,28.5 \mathrm{ft}^{3} / \mathrm{s}$.

05407015 Mi11 Creek near Muscoda, Wis.

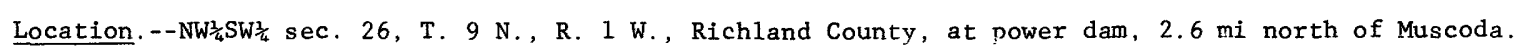

Drainage area. $--91.7 \mathrm{mi}^{2}$.

Tributary to. - -Wisconsin River.

Type of site.--Miscellaneous site.

Discharge measurement.--Aug. $20,1914,43.7 \mathrm{ft}^{3} / \mathrm{s}$.

054070182 Blue River near Montfort, Wis.

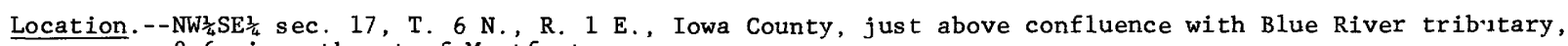
$2.6 \mathrm{mi}$ northeast of Montfort.

Drainage area. $--4.88 \mathrm{mi}^{2}$. Tributary to. --Wisconsin River.

Type of site.--Miscellaneous site.

Discharge measurement. --Oct. $19,1972,1.47 \mathrm{ft}^{3} / \mathrm{s}$.

054070185 Blue River tributary near Montfort, Wis.

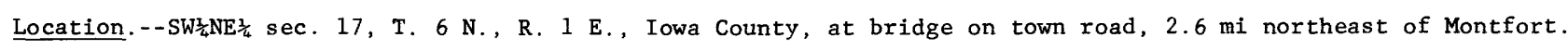
Drainage area. $--3.22 \mathrm{mi}^{2}$. Tributary to. --Blue River.

Type of site.--Miscellaneous site.

Discharge measurement.--0ct. $19,1972,0.18 \mathrm{ft}^{3} / \mathrm{s}$.

054070187 Blue River tributary near Highland, Wis.

Location. - $-\mathrm{NW} \frac{1}{4} \mathrm{SW} \frac{1}{4}$ sec. $8, \mathrm{~T} .6 \mathrm{~N} ., \mathrm{R} .1 \mathrm{~W}$, Iowa County, at culvert on town road, $3.2 \mathrm{mi}$ southwest of Highland. Drainage area. $--3.93 \mathrm{mi}^{2}$. Tributary to.--Blue River.

Type of site.--Miscellaneous site.

Discharge measurement.--Oct. 19, 1972, $0.039 \mathrm{ft}^{3} / \mathrm{s}$.

05407020 Blue River tributary at Montfort, Wis.

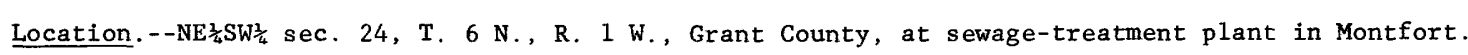

Drainage area. $-0.58 \mathrm{mi}^{2}$. Tributary to.--Blue River.

Type of site.--Miscellaneous site.

Discharge measurements.- - July 7, 1972, $0.550 \mathrm{ft}_{3}^{3} / \mathrm{s} ;$ June $28,1973,1.26 \mathrm{ft} / \mathrm{s} ; \mathrm{Aug} .23,1973,1.12 \mathrm{f} \mathrm{H}^{3} / \mathrm{s} ;$

Low-f1ow frequency. $--Q_{7,2}=0.36 \mathrm{ft}^{3} / \mathrm{s}, Q_{7,10}=0.26 \mathrm{ft}^{3} / \mathrm{s}$.

Basis of estimate.--Correlated with Platte River near Rockville using 4 discharge measurements.

Accuracy. $--\mathrm{SE}_{7,10}=19$ percent (average value for basin). 
05407021 Blue River near Highland, Wis.

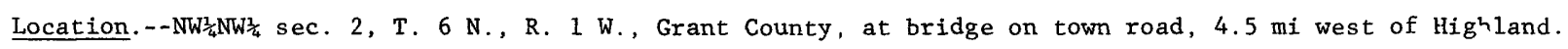
Drainage area. $--29.8 \mathrm{mi}^{2}$. Tributary to.--Wisconsin River.

Type of site.--Miscellaneous site.

Discharge measurements.--Oct. $19,1972,12.5 \mathrm{ft}^{3} / \mathrm{s} ; \mathrm{July} 22,1976,14.2 \mathrm{ft}^{3} / \mathrm{s}$.

054070215 Big Rock Branch near Highland, Wis.

Location. - - NE $\frac{1}{4} \mathrm{SE} \frac{1}{4}$ sec. 26, T. 7 N., R. 1 W., Grant County, at bridge on town road, $3.8 \mathrm{mi}$ west of Highland. Drainage area. $--9.42 \mathrm{mi}^{2}$. Tributary to. --Blue River.

Type of site.--Miscellaneous site.

Discharge measurement. - - Oct. $19,1972,0.61 \mathrm{ft}^{3} / \mathrm{s}$.

05407023 Blue River near Muscoda, Wis.

Location.- $-\mathrm{NW} \frac{1}{4} \mathrm{SW} \frac{3}{4} \mathrm{sec} .10$, T. 7 N., R. 1 W., Grant County, at bridge on town road, $6.7 \mathrm{mi}$ south of M scoda. Drainage area. $--50.0 \mathrm{mi}^{2}$. Tributary to. - Wisconsin River.

Type of site.--Miscellaneous site.

Discharge measurement.--Oct. 17, 1972, $20.7 \mathrm{ft}^{3} / \mathrm{s}$.

05407024 Sixmile Branch near Muscoda, Wis.

Location. - $-\mathrm{SW}_{\frac{3}{4}} \mathrm{SW} \frac{1}{4}$ sec. 2, T. 7 N., R. 1 W., Grant County, at bridge on town road, 5.8 mi south of Muscoda.

Drainage area. $--11.3 \mathrm{mi}^{2}$. Tributary to.--Blue River.

Type of site.--Miscellaneous site.

Discharge measurement. - - Oct. 19, 1972, $2.68 \mathrm{ft}^{3} / \mathrm{s}$.

05407025 Big Spring Branch at Highland, Wis.

Location. $--\mathrm{NE}_{4} \mathrm{SE} \frac{3}{4}$ sec. $29, \mathrm{~T} .7 \mathrm{~N} .$, R. 1 E., Iowa County, at sewage-disposal pond, at Highland.

Drainage area. $-0.68 \mathrm{mi}^{2}$. Tributary to.--Sixmile Branch.

Type of site.--Miscellaneous site.

Discharge measurements.--Aug, 23, 1973,0.30 $\mathrm{ft}^{3} / \mathrm{s} ;$ Oct. $8,1975,0.007 \mathrm{ft}^{3} / \mathrm{s}$.

05407026 Big Spring Branch near Muscoda, Wis.

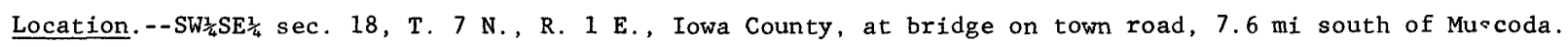
Drainage area. $--7.26 \mathrm{mi}^{2}$. Tributary to.--Sixmile Branch.

Type of site.--Miscellaneous site.

Discharge measurement. - - Oct. 19, 1972, $1.42 \mathrm{ft}^{3} / \mathrm{s}$.

05407027 Big Spring Branch near Muscoda, Wis.

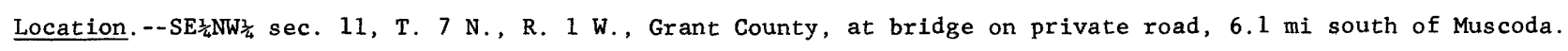
Drainage area. $--12.0 \mathrm{mi}^{2}$. Tributary to. --Sixmile Branch.

Type of site.--Miscellaneous site.

Discharge measurements.--Oct. $19,1972,4.39 \mathrm{ft}^{3} / \mathrm{s} ;$ July $22,1976,5.13 \mathrm{ft}^{3} / \mathrm{s}$. 
05407030 Blue River near Blue River, Wis.

Location.
Blue River.

Drainage area. $--79.6 \mathrm{mi}^{2}$. Tributary to. --Wisconsin River.

Type of site.--Miscellaneous site.

Discharge measurements.--Aug, $19,1970,31.3 \mathrm{ft}^{3} / \mathrm{s} ;$ Oct. 17, 1972, 35.5 ft $3 / \mathrm{s} ; \mathrm{Ju} 1 \mathrm{y} 22,1976,40.0 \mathrm{ft} / \mathrm{s}$.

05407037 Fennimore Fork near Castle Rock, Wis.

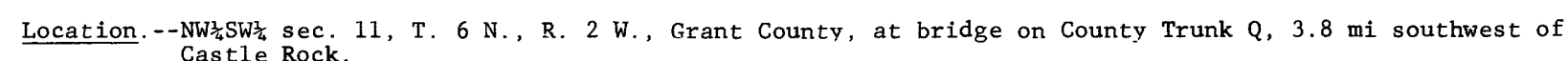
Drainage area. $--7.10 \mathrm{mi}^{2}$. Tributary to.--Blue River.

Type of site.--Miscellaneous site.

Discharge measurement.--Oct. $18,1972,1.27 \mathrm{ft}^{3} / \mathrm{s}$.

05407040 Fennimore Fork near Castle Rock, Wis.

Location.--NE考SWh sec. 36, T. 7 N., R. 2 W., Grant County, at bridge on County Trunk Q, 1.7 mi southwest of Drainage area. $--23.3 \mathrm{mi}^{2}$. Tributary to.--Blue River.

Type of site.--Miscellaneous site.

Discharge measurements.--Oct, $18,1972,1.38 \mathrm{ft}^{3} / \mathrm{s} ; \mathrm{July} 22,1976,0.60 \mathrm{ft} / \mathrm{s}$.

05407042 Fennimore Fork tributary near Castle Rock, Wis.

Location.--
Rock. Drainage area. $--15.5 \mathrm{mi}^{2}$. Tributary to. --Fennimore Fork.

Type of site.--Miscellaneous site.

Discharge measurement.--Oct. $18,1972,4.66 \mathrm{ft}^{3} / \mathrm{s}$.

05407044 Fennimore Fork tributary at Castle Rock, Wis .

Location. $-\mathrm{NE}_{\frac{1}{4}} \mathrm{NE}_{\frac{1}{4}} \mathrm{sec} .31, \mathrm{~T} .7 \mathrm{~N} ., \mathrm{R} .1 \mathrm{~W}$. , Grant County, at bridge on town road, at Castle Rock.

Drainage area. $--3.73 \mathrm{mi}^{2}$.

Tributary to.--Fennimore Fork.

Type of site.--Miscellaneous site.

Discharge measurement. - Oct. 18, 1972, $0.12 \mathrm{ft}^{3} / \mathrm{s}$.

05407045 Fennimore Fork tributary near Blue River, Wis.

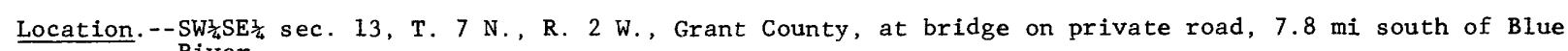
River.

Drainage area. $--10.7 \mathrm{mi}^{2}$. Tributary to.--Fennimore Fork.

Type of site.-Miscellaneous site.

Discharge measurement.--Oct. $18,1972,3.69 \mathrm{ft}^{3} / \mathrm{s}$.

05407046 Fennimore Fork near Blue River, Wis.

Location. -- SW $\frac{1}{4} \mathrm{NW} \frac{1}{4} \mathrm{sec} .18, \mathrm{~T} .7 \mathrm{~N} ., \mathrm{R} .1 \mathrm{~W} .$, Grant County, at bridge on town road, $7.3 \mathrm{mi}$ south of Blue River. Drainage area. $--61.2 \mathrm{mi}^{2}$. Tributary to.--Blue River.

Type of site.--Miscellaneous site.

Discharge measurement.--Oct. 18, 1972, $25.1 \mathrm{ft}^{3} / \mathrm{s}$. 
Table 1.--Low-flow characteristics for sites in the lower Wisconsin River basin--Continued

05407048 Fennimore Fork near Blue River, Wis.

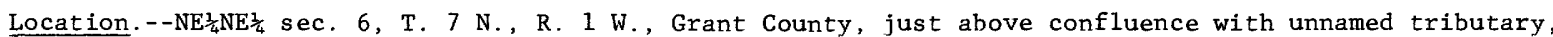

$5.2 \mathrm{mi}$ south of Blue River.

Drainage area. $--68.8 \mathrm{mi}^{2}$. Tributary to.--Blue River.

Type of site.--Miscellaneous site.

Discharge measurement. - - Oct. $18,1972,31.6 \mathrm{ft}^{3} / \mathrm{s}$.

05407049 Fennimore Fork tributary near Blue River, Wis.

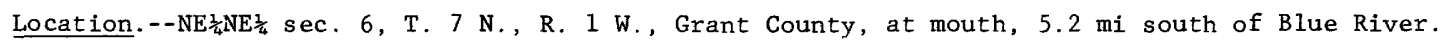

Drainage area. $--8.34 \mathrm{mi}^{2}$.

Tributary to. --Fennimore Fork

Type of site.--Miscellaneous site.

Discharge measurement.--Oct. $18,1972,2.26 \mathrm{ft}^{3} / \mathrm{s}$.

05407050 Fennimore Fork near Blue River, Wis.

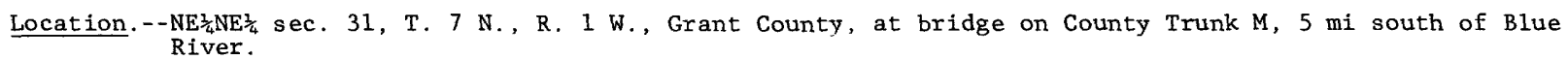

Drainage area. $--77.2 \mathrm{mi}^{2}$. Tributary to.--Blue River.

Type of site.--Low-flow partial-record station.

Minimum discharge measured. $--19.3 \mathrm{ft}^{3} / \mathrm{s}$, Sept. 6, 1967.

Low-flow frequency. $-Q_{7,2}=19 \mathrm{ft}^{3} / \mathrm{s}, Q_{7,10}=14 \mathrm{ft}^{3} / \mathrm{s}$.

Basis of estimate.--Correlated with Platte River near Rockville using 10 discharge measurements made in the Accuracy. $--\mathrm{SE}_{7,10}=18$ percent.

05407054 Blue River near Boscobel, Wis.

Location.-- $\mathrm{SE}_{\frac{1}{4} \mathrm{SW}} \frac{1}{4} \mathrm{sec} .29, \mathrm{~T} .8 \mathrm{~N} ., \mathrm{R} .1 \mathrm{~W} .$, Grant County, at bridge on town road, $4.2 \mathrm{mi}$ southeast of Blue River.

Drainage area. $--161 \mathrm{mi}^{2}$. Tributary to. --Wisconsin River.

Type of site.--Miscellaneous site.

Discharge measurement.--July $27,1964,51.2 \mathrm{ft}^{3} / \mathrm{s}$.

05407061 Sand Branch near Muscoda, Wis.

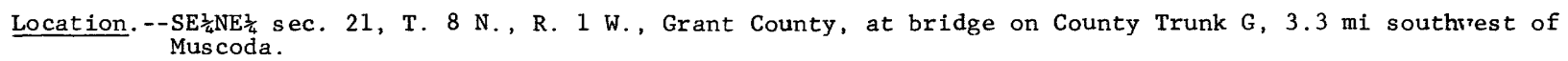
Drainage area. $--8.42 \mathrm{mi}^{2}$. Tributary to.--Blue River.

Type of site.--Miscellaneous site.

Discharge measurements.--Aug. 19, $1970,1.68 \mathrm{ft}^{3} / \mathrm{s} ;$ Oct. $17,1972,2.86 \mathrm{ft}^{3} / \mathrm{s} ; \mathrm{July} 23,1976,2.80 \mathrm{ft} / \mathrm{s}$.

05407062 Blue River tributary near Muscoda, Wis.

Location. - - $-\mathrm{SW}_{4} \mathrm{NE} \frac{1}{4}$ sec. 15, T. 8 N., R. 1 W., Grant County, at bridge on County Trunk G, 2.0 mi southrest of Drainage area. $--7.82 \mathrm{mi}^{2}$. Tributary to.--Blue River.

Type of site,--Miscellaneous site.

Discharge measurement.--0ct. $17,1972,0 \mathrm{ft}^{3} / \mathrm{s}$. 
05407063 Blue River near Blue River, Wis.

Location. - $-\mathrm{NW} \frac{1}{4} \mathrm{NW} \frac{2}{4} \mathrm{sec} .16$, T. $8 \mathrm{~N} .$, R. I W., Grant County, at bridge on town road, 3.2 mi east of Blue River. Drainage area. $--186 \mathrm{mi}^{2}$. Tributary to. --Wisconsin River.

Type of site.--Miscellaneous site.

Discharge measurements.--July $27,1964,56.4 \mathrm{ft}_{3}^{3} / \mathrm{s} ;$ July $30,1965,46.7 \mathrm{ft}^{3} / \mathrm{s} ;$ Oct. $17,1972,77.1 \mathrm{ft} / \mathrm{s}$; July 23, $1976,74.6 \mathrm{ft} / \mathrm{s}$.

Low-flow frequency. $-Q_{7,2}=58 \mathrm{ft}^{3} / \mathrm{s}, Q_{7,10}=47 \mathrm{ft}^{3} / \mathrm{s}$.

Basis of estimate.--Correlated with Black Earth Creek at Black Earth using 4 discharge measurements.

Accuracy. $--\mathrm{SE}_{7,10}=19$ percent (average value for basin).

05407064 Blue River at Blue River, Wis.

Location.-- $-\mathrm{NE} \frac{1}{4} \mathrm{NW}_{\frac{2}{4}} \mathrm{sec} .12, \mathrm{~T} .8 \mathrm{~N} .$, R. $2 \mathrm{~W}$, Grant County, at bridge on State Highway 133, at Blue River. Drainage area. $--193 \mathrm{mi}^{2}$. Tributary to. - -Wisconsin River.

Type of site.--Miscellaneous site.

Discharge measurement.--0ct. $17,1972,79.3 \mathrm{ft}^{3} / \mathrm{s}$.

05407070 Knapp Creek at Excelsior, Wis.

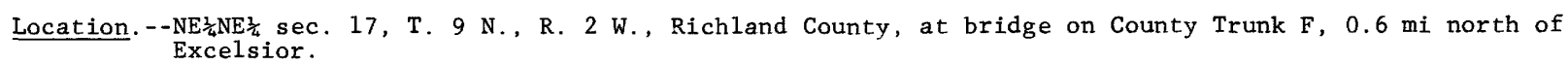
Drainage area. $--53.7 \mathrm{mi}^{2}$. Tributary to. - Wisconsin River.

Type of site.--Miscellaneous site.

Discharge measurements. --July 10, 1963, $20.9 \mathrm{ft}^{3} / \mathrm{s} ;$ July $21,1964,18.6 \mathrm{ft}^{3} / \mathrm{s}$.

05407071 Knapp Creek near Excelsior, Wis.

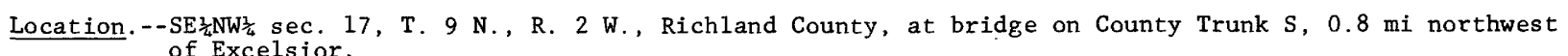
Drainage area. $--54.2 \mathrm{mi}^{2}$. Tributary to. - -Wisconsin River.

Type of site.--Miscellaneous site.

Discharge measurement. --Aug. 18, 1970, $20.9 \mathrm{ft}^{3} / \mathrm{s}$.

05407078 West Fork Knapp Creek near Excelsior, Wis.

Location. -- $\mathrm{SW}_{\frac{1}{4}} \mathrm{SEE}_{\frac{1}{4}} \mathrm{sec} .7$, T. 9 N., R. 2 W., Richland County, at bridge on County Trunk S, 1.5 mi northwest of Excelsior.

Drainage area. $-17.1 \mathrm{mi}^{2}$. Tributary to. --Knapp Creek.

Type of site.--Miscellaneous site.

Discharge measurement.--Aug. $18,1970,6.99 \mathrm{ft}^{3} / \mathrm{s}$.

05407080 Knapp Creek near Westport, Wis.

Location.-- $-\mathrm{SE}_{\frac{1}{4} \mathrm{SW} \frac{1}{4} \mathrm{sec} .}$ 32, T. 9 N., R. 2 W., Richland County, at bridge on State Highway $60,0.7$ mi west of Drainage area. $--85.1 \mathrm{mi}^{2}$. Tributary to. - Wisconsin River.

Type of site.--Low-flow partial-record station.

Minimum discharge measured. $--25.7 \mathrm{ft}^{3} / \mathrm{s}$, July $30,1965$.

Low-flow frequency. $--Q_{7,2}=28 \mathrm{ft}^{3} / \mathrm{s}, Q_{7,10}=23 \mathrm{ft}^{3} / \mathrm{s}$. Basis of estimate. - - Correlated with Kickapoo River at Steuben using 7 discharge measurements made in the

Accuracy. $--\mathrm{SE}_{7,10}=12$ percent. 
05407100 Richland Creek near Plugtown, Wis.

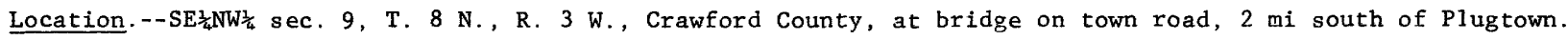
Drainage area. $--19.2 \mathrm{mi}^{2}$.

Tributary to. --Wisconsin River.

Type of site.--Low-flow partial-record station.

Minimum discharge measured. $--5.69 \mathrm{ft}^{3} / \mathrm{s}$, Sept. 6, 1967.

Low-flow frequency. $--Q_{7,2}=6.2 \mathrm{ft}^{3} / \mathrm{s}, Q_{7,10}=4.8 \mathrm{ft}^{3} / \mathrm{s}$.

Basis of estimate.--Correlated with Kickapoo River at Steuben using 19 discharge measurements made ir the period 1961-75.

Accuracy. $--\mathrm{SE}_{7,10}=12$ percent.

05407130 Saunders Creek at Boscobel, Wis.

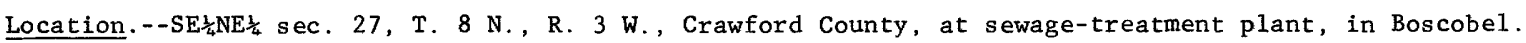

Drainage area. $--17.1 \mathrm{mi}^{2}$. Tributary to. --Wisconsin River.

Type of site. --Miscellaneous site.

Discharge measurements.--July $6,1972,4.88 \mathrm{ft}_{3}^{3} / \mathrm{s} ;$ June $27,1973,13.9 \mathrm{ft}^{3} / \mathrm{s} ;$ Aug. $23,1973,12.5 \mathrm{ft} 2 / \mathrm{s}$; Oct. $8,1975,6.91 \mathrm{ft}^{3} / \mathrm{s}$.

Low-flow frequency. $-Q_{7,2}=3.4 \mathrm{ft}^{3} / \mathrm{s}, Q_{7,10}=2.4 \mathrm{ft}^{3} / \mathrm{s}$.

Basis of estimate. --Correlated with Grant River at Burton using 4 discharge measurements.

Accuracy.--SE $7,10=19$ percent (average value for basin).

05407200 Crooked Creek near Boscobel, Wis. Location.-- $-\mathrm{SW}_{\frac{1}{4}} \mathrm{SW}_{\frac{1}{4}} \mathrm{sec} .2$, T. 7 N., R. 3 W., Grant County, at bridge on U.S. Highway $61,1.6 \mathrm{mi}$ south of

Drainage area. $--12.9 \mathrm{mi}^{2}$. Tributary to. - -Wisconsin River.

Type of site.--Low-flow partial-record stations.

Minimum discharge measured. $--5.07 \mathrm{ft}^{3} / \mathrm{s}$, Sept. 6, 1967.

Low-flow frequency. $--Q_{7,2}=6.0 \mathrm{ft}^{3} / \mathrm{s}, Q_{7,10}=4.9 \mathrm{ft}^{3} / \mathrm{s}$.

Basis of estimate.--Correlated with Grant River at Burton using 18 discharge measurements made in the period 1961-75.

Accuracy. $-\mathrm{SE}_{7,10}=8$ percent.

05407250 Big Green River near Wauzeka, Wis.

Location.-- $\mathrm{SE}_{\frac{1}{4}} \mathrm{SW}_{\frac{1}{4}} \mathrm{sec} .6$, T. $6 \mathrm{~N} .$, R. $3 \mathrm{~W}$., Grant County, at bridge on town road, 7.0 mi southeast of Wauzeka.

Drainage area. $--22.5 \mathrm{mi}^{2}$.

Tributary to. --Wisconsin River.

Type of site.--Miscellaneous site.

Discharge measurement. --Aug. $18,1970,5.97 \mathrm{ft}^{3} / \mathrm{s}$.

05407260 Big Green River near Wauzeka, Wis .

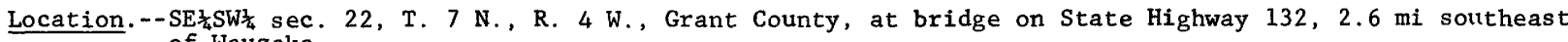
of Wauzeka.

Drainage area. $--48.4 \mathrm{mi}^{2}$.

Tributary to.--Wisconsin River.

Type of site.--Miscellaneous site.

Discharge measurement.--Aug. 18, 1970, $20.2 \mathrm{ft}^{3} / \mathrm{s}$. 
05407280 Little Green River near Wauzeka, Wis.

Location.--SW $\frac{1}{4} \mathrm{NW} \frac{1}{4} \mathrm{sec} .27$, T. 7 N., R. 4 W., Grant County, at bridge on County Trunk C, 2.8 mi southeast of Wauzeka.

Drainage area. $--16.5 \mathrm{mi}^{2}$. Tributary to. --Big Green River.

Type of site.--Miscellaneous site.

Discharge measurement.--Aug. 18, 1970,6.56 $\mathrm{ft}^{3} / \mathrm{s}$.

05407360 Kickapoo River at Wilton, Wis.

Location.--NW $\frac{1}{4} \mathrm{NW} \frac{1}{4}$ sec. 5, T. 15 N., R. 1 W., Monroe County, at bridge on State Highway 131, at Wilton.

Drainage area. $--34.9 \mathrm{mi}^{2}$.

Tributary to. - -Wisconsin River.

Type of site.--Miscellaneous site.

Discharge measurements.--Aug. $18,1970,7.88 \mathrm{ft}^{3} / \mathrm{s} ;$ July $6,1972,9.66 \mathrm{ft}^{3} / \mathrm{s} ;$ June $26,1973,25.8 \mathrm{ft} / \mathrm{s} ;$ Low-flow frequency. $-\mathrm{Q}_{7,2}=6.6 \mathrm{ft}^{3} / \mathrm{s}, \mathrm{Q}_{7,10}=4.8 \mathrm{ft}^{3} / \mathrm{s}$.

Basis of estimate.--Correlated with Kickapoo River at La Farge using 4 discharge measurements.

Accuracy. $--\mathrm{SE}_{7,10}=19$ percent (average value for basin).

05407380 Poe Creek near Wilton, Wis.

Location. - - $-\mathrm{SE}_{\frac{1}{4}} \mathrm{SE}_{\frac{1}{4}} \mathrm{sec} .7$, T. $15 \mathrm{~N} .$, R. $1 \mathrm{~W}$, Monroe County, at bridge on County Trunk Z, $1.9 \mathrm{mi}$ south of Wilton.

Drainage area. $--9.87 \mathrm{mi}^{2}$. Tributary to. --Kickapoo River.

Type of site.--Miscellaneous site.

Discharge measurement.--Aug. 18, 1970, $1.83 \mathrm{ft}^{3} / \mathrm{s}$.

05407400 Morris Creek tributary near Norwalk, Wis.

Location.--NE $\frac{1}{4} N W \frac{2}{4}$ sec. 21, T. 16 N., R. 2 W., Monroe County, at bridge on County Trunk T, 2 mi north of Norwalk.

Drainage area. $--4.59 \mathrm{mi}^{2}$.

Tributary to. --Morris Creek.

Type of site.--Low-flow partial-record station.

Minimum discharge measured. $--0.58 \mathrm{ft}^{3} / \mathrm{s}$, July $11,1963$.

Low-flow frequency. $-Q_{7,2}=0.78 \mathrm{ft}^{3} / \mathrm{s}, Q_{7,10}=0.49 \mathrm{ft}^{3} / \mathrm{s}$. Basis of estimate.--Correlated with La Crosse River near West Salem using 20 measurements made in ths period

Accuracy. $--\mathrm{SE}_{7,10}=12$ percent.

05407410 Morris Creek tributary at Norwalk, Wis.

Location.--SW $\frac{1}{4} \mathrm{SE}_{\frac{1}{4}}$ sec. 28, T. 16 N., R. 2 W., Monroe County, at bridge on County Trunk U, in Norwalk.

Drainage area. $--18.1 \mathrm{mi}^{2}$.

Tributary to. --Morris Creek.

Type of site.--Miscellaneous site.

Discharge measurement. --July $6,1972,4.98 \mathrm{ft}^{3} / \mathrm{s}$. 
Table 1.--Low-flow characteristics for sites in the lower Wisconsin River basin--Continued

05407411 Morris Creek tributary at Norwalk, Wis.

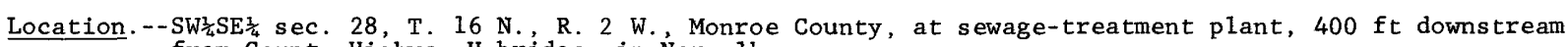
from County Highway U bridge, in Norwalk.

Drainage area. $--19.9 \mathrm{mi}^{2}$. Tributary to. --Morris Creek.

Type of site.--Miscellaneous site.

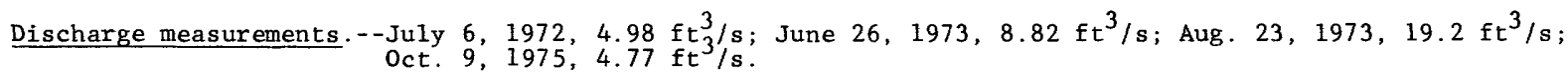

Low-flow frequency. $-Q_{7,2}=2.8 \mathrm{ft}^{3} / \mathrm{s}, Q_{7,10}=1.8 \mathrm{ft}^{3} / \mathrm{s}$.

Basis of estimate.--Correlated with Baraboo River at Baraboo using 4 discharge measurements.

Accuracy.- $-\mathrm{SE}_{7,10}=19$ percent (average value for basin).

05407470 Kickapoo River at Ontario, Wis .

Location.-- $-\mathrm{SW}_{\frac{1}{4}} \mathrm{NW} \frac{1}{4} \mathrm{sec} .2, \mathrm{~T} .14 \mathrm{~N}, \mathrm{R} .2 \mathrm{~W}$. , Vernon County, at bridge on State Highway $33,0.1 \mathrm{mi}$ upstream from sewage-treatment plant, in Ontario.

Drainage area. $--117 \mathrm{mi}^{2}$. Tributary to. --Wisconsin River.

Type of site.--Miscellaneous site.

Minimum discharge measured. $--29.2 \mathrm{ft}^{3} / \mathrm{s}$, Aug. 18, 1970.

Low-flow frequency. $-Q_{7,2}=28 \mathrm{ft}^{3} / \mathrm{s}, Q_{7,10}=21 \mathrm{ft}^{3} / \mathrm{s}$.

Basis of estimate.--Correlated with Kickapoo River at La Farge using 5 dischage measurements.

Accuracy. $--\mathrm{SE}_{7,10}=19$ percent (average value for basin).

05407490 Brush Creek at Ontario, Wis. .

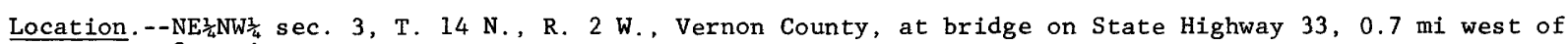
Ontario.

Drainage area. $--31.2 \mathrm{mi}^{2}$. Tributary to. --Kickapoo River.

Type of site.--Miscellaneous site.

Discharge measurement.--Aug. 18, 1970, $10.5 \mathrm{ft}^{3} / \mathrm{s}$.

05407495 Brush Creek at Ontario, Wis.

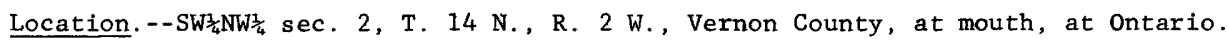

Drainage area. $--32.0 \mathrm{mi}^{2}$. Tributary to. --Kickapoo River.

Type of site.--Miscellaneous site.

Discharge measurements. --July $6,1972,9.73 \mathrm{ft}^{3} / \mathrm{s} ;$ Oct. $8,1975,13.1 \mathrm{ft}^{3} / \mathrm{s}$.

05407500 Kickapoo River at Ontario, Wis .

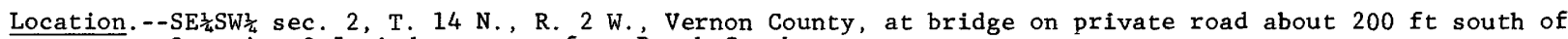
Ontario, 0.5 mi downstream from Brush Creek.

Drainage area. $--151 \mathrm{mi}^{2}$.

Tributary to.--Wisconsin River.

Type of site.--Gaging station.

Minimum discharge measured. $--32.4 \mathrm{ft}^{3} / \mathrm{s}$, Aug. 26, 1969.

Low-flow frequency. - $-Q_{7,2}=37 \mathrm{ft}^{3} / \mathrm{s}, Q_{7,10}=26 \mathrm{ft}^{3} / \mathrm{s}$.

Period of record.--1938-39, July 1973 to September 1975.

Basis of estimate. - - Correlated with Kickapoo River at La Farge using 12 mean daily discharges recorded during water year 1939 and 2 discharge measurements made in 1969.

Accuracy. $--\mathrm{SE}_{7,10}=19$ percent. 


\section{Billings Creek near Ontario, Wis.}

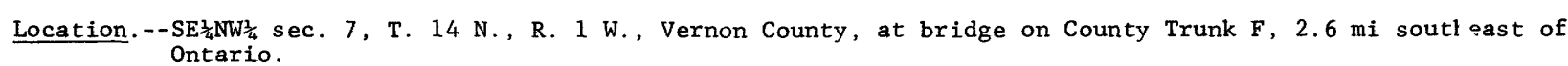
Drainage area. $--20.7 \mathrm{mi}^{2}$. Tributary to. --Kickapoo River.

Type of site.--Miscellaneous site.

Discharge measurements.--Jan. 3, 1962, $10.1 \mathrm{ft}^{3} / \mathrm{s} ;$ June 9, 1964, 6.78 ft $/ \mathrm{s} ; \mathrm{Aug} .18,1970,7.85 \mathrm{ft} / \mathrm{s}$. Low-flow frequency. $--Q_{7,2}=6.5 \mathrm{ft}^{3} / \mathrm{s}, Q_{7,10}=5.2 \mathrm{ft}^{3} / \mathrm{s}$.

Basis of estimate.--Correlated with Kickapoo River at La Farge using 3 discharge measurements.

Accuracy. $--\mathrm{SE}_{7,10}=19$ percent (average value for basin).

\section{Billings Creek near Ontario, Wis.}

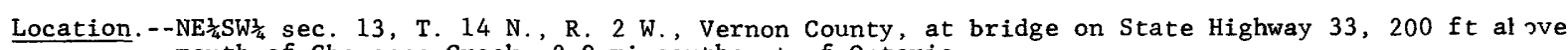
mouth of Cheyenne Creek, $2.9 \mathrm{mi}$ southeast of Ontario.

Drainage area. $--23.0 \mathrm{mi}^{2}$. Tributary to.--Kickapoo River.

Type of site.-Miscellaneous site.

Minimum discharge measured. $--8.15 \mathrm{ft}^{3} / \mathrm{s}$, Aug. 10, 1964.

Low-flow frequency. $-Q_{7,2}=8.0 \mathrm{ft}^{3} / \mathrm{s}, Q_{7,10}=5.9 \mathrm{ft}^{3} / \mathrm{s}$.

Basis of estimate.--Correlated with Kickapoo River at La Farge using 11 discharge measurements.

Accuracy. $--\mathrm{SE}_{7,10}=19$ percent.

05407800 Warner Creek near La Farge, Wis.

Location.-- $-\mathrm{SW}_{\frac{3}{4} \mathrm{NW} \frac{3}{4}}$ sec. 35, T. $14 \mathrm{~N} .$, R. 2 W., Vernon County, at bridge on County Trunk P, 5.7 mi northeast
of La Farge. Drainage area. $--24.9 \mathrm{mi}^{2}$. Tributary to.--Kickapoo River.

Type of site.--Miscellaneous site.

Discharge measurement. --Aug. $18,1970,10.8 \mathrm{ft}^{3} / \mathrm{s}$.

05407900 Weister Creek near La Farge, Wis.

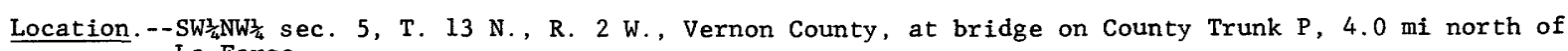
La Farge.

Drainage area. $-18.0 \mathrm{mi}^{2}$.

Tributary to.--Kickapoo River.

Type of site.--Miscellaneous site.

Discharge measurement.--Aug. $18,1970,6.95 \mathrm{ft}^{3} / \mathrm{s}$.

\section{Kickapoo River near La Farge, Wis.}

Location. - $-\mathrm{NE} \frac{1}{4} \mathrm{NE} \frac{1}{4} \mathrm{sec} .20, \mathrm{~T} .13 \mathrm{~N} .$, R. $2 \mathrm{~W}$, Vernon County, at bridge on State Highway 131 , $1.4 \mathrm{mi}$ north of La Farge.

Drainage area. $--262 \mathrm{mi}^{2}$.

Tributary to.--Wisconsin River.

Type of site.--Miscellaneous site.

Discharge measurement.--0ct. 6, $1971,129 \mathrm{ft}^{3} / \mathrm{s}$. 
05408000 Kickapoo River at La Farge, Wis.

Location. - -SE $\frac{1}{4} N W \frac{1}{4}$ sec. 29, T. 13 N., R. $2 \mathrm{~W}$, Vernon County, on left bank $10 \mathrm{ft}$ upstream from bridge on State Highway 82 , in La Farge, 0.3 mi upstream from otter Creek, 1 mi downstream from powerplant.

Drainage area. $--266 \mathrm{mi}^{2}$. Tributary to.--Wisconsin River.

Type of site.--Gaging station.

Period of record.--October 1938 through September 1975.

Average discharge. --37 years, $170 \mathrm{ft}^{3} / \mathrm{s}$.

Extremes.--Maximum discharge, $9,910 \mathrm{ft}^{3} / \mathrm{s} \mathrm{Feb.} \mathrm{9,} \mathrm{1966;} \mathrm{minimum} \mathrm{discharge,} 1.8 \mathrm{ft}^{3} / \mathrm{s} \mathrm{Mar} .24,1961$.

Period Magnitude and frequency of annual low flow

of con- Discharge, in cubic feet per second, for

secutive indicated recurrence interval, in years

days

\begin{tabular}{|r|rrrrrr|}
\hline & 2 & 5 & 10 & 20 & 50 & 100 \\
\cline { 2 - 6 } 14 & 77 & 67 & 63 & 60 & 57 & 56 \\
30 & 80 & 70 & 66 & 63 & 60 & 59 \\
60 & 86 & 75 & 71 & 68 & 65 & 63 \\
90 & 92 & 80 & 75 & 71 & 68 & 66 \\
& 100 & 86 & 79 & 74 & 69 & 66 \\
\hline
\end{tabular}

Accuracy. $--\mathrm{SE}_{7,2}=4$ percent, $\mathrm{SE}_{7,10}=4$ percent.

05408200 Bear Creek near La Farge, Wis.

Location. - $-\mathrm{SW}_{\frac{1}{4}} \mathrm{NE} \frac{1}{4} \mathrm{sec} .28, \mathrm{~T} .13 \mathrm{~N} ., \mathrm{R} .2 \mathrm{~W}$. , Vernon County, $0.6 \mathrm{mi}$ east of La Farge, 0.7 mi downstream from mouth of North Bear Creek.

Drainage area. $--23.4 \mathrm{mi}^{2}$.

Tributary to.--Kickapoo River.

Type of site.--Miscellaneous site.

Discharge measurement.--Aug. 18, $1970,7.92 \mathrm{ft}^{3} / \mathrm{s}$.

05408300 Camp Creek near Viola, Wis.

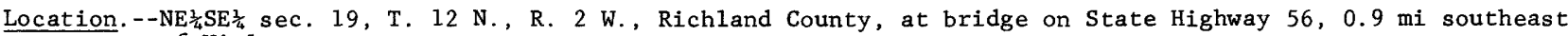
of Viola.

Drainage area. $--16.0 \mathrm{mi}^{2}$.

Tributary to. --Kickapoo River.

Type of site.--Miscellaneous site.

Discharge measurement.--Aug. $19,1970,9.27 \mathrm{ft}^{3} / \mathrm{s}$.

05408500 Knapp Creek near Bloomingdale, Wis.

Location. - -NE $\frac{1}{4} N W \frac{1}{4}$ sec. 30, T. 14 N., R. 3 W., Vernon County, on right bank, 0.4 mi upstream from confluence with West Fork Kickapoo River, $1.7 \mathrm{mi}$ north of Bloomingdale, $4 \mathrm{mi}$ east of Westby.

Drainage area. $--8.44 \mathrm{mi}^{2}$.

Tributary to.--West Fork Kickapoo River.

Type of site.--Gaging station.

Period of record.--October 1954 through September 1969.

Average discharge. --15 years, $4.96 \mathrm{ft}^{3} / \mathrm{s}$.

Extremes. --Maximum discharge, 3,710 $\mathrm{ft}^{3} / \mathrm{s}$ Aug. 26, 1959; minimum discharge, $0.4 \mathrm{ft} \mathrm{f}^{3} / \mathrm{s} \mathrm{Nov}$. $28,1966$.

Period Magnitude and frequency of annual low flow

of con- Discharge, in cubic feet per second, for

secutive Discharge, in cubic feet per second, for

days

\begin{tabular}{|c|c|c|c|c|}
\hline & 2 & 5 & 10 & 20 \\
\hline $\begin{array}{r}7 \\
14 \\
30 \\
60 \\
90\end{array}$ & $\begin{array}{l}1.3 \\
1.4 \\
1.8 \\
2.0 \\
2.2\end{array}$ & $\begin{array}{l}0.96 \\
1.1 \\
1.4 \\
1.5 \\
1.6\end{array}$ & $\begin{array}{l}0.81 \\
.88 \\
1.1 \\
1.2 \\
1.4\end{array}$ & $\begin{array}{r}0.70 \\
.75 \\
.95 \\
1.1 \\
1.2\end{array}$ \\
\hline
\end{tabular}

Duration table of daily flow

Discharge, in cubic feet per second, which was exceeded for indicated percent of time

$\begin{array}{lllllllll}\text { Percent } & 2 & 5 & 10 & 20 & 30 & 40 & 59\end{array}$

\begin{tabular}{lllllllll}
$\mathrm{ft}^{3} / \mathrm{s}$ & 34 & 12 & 6.4 & 3.8 & 3.0 & 2.6 & 2.3 \\
\hline
\end{tabular}

$\begin{array}{llllllll}\mathrm{ft}^{3} / \mathrm{s} & 2.1 & 1.9 & 1.7 & 1.5 & 1.3 & 1.1 & 0.5\end{array}$

Accuracy. $--\mathrm{SE}_{7,2}=8$ percent, $\mathrm{SE}_{7,10}=16$ percent. 
05408700 West Fork Kickapoo River near Viroqua, Wis.

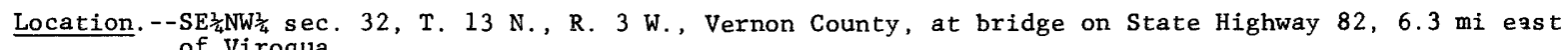
of Viroqua.

Drainage area. $--63.5 \mathrm{mi}^{2}$. Tributary to.--Kickapoo River.

Type of site.--Low-flow partial-record station.

Minimum discharge measured. $--17.8 \mathrm{ft}^{3} / \mathrm{s}$, Aug. 19, 1964.

Low-flow frequency. $--Q_{7,2}=15.3 \mathrm{ft}^{3} / \mathrm{s}, 0_{7,10}=7.6 \mathrm{ft}^{3} / \mathrm{s}$.

Basis of estimate.--Correlated with Knapp Creek near Bloomingdale using 10 discharge measurements made in the period 1963-75.

Accuracy. $-\mathrm{SE}_{7,10}=19$ percent.

05408800 Bishops Branch near Viroqua, Wis.

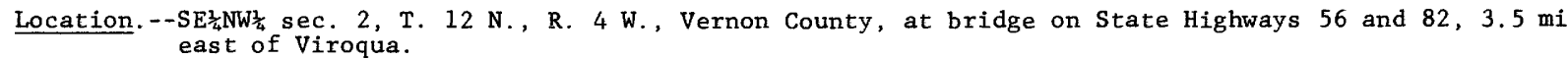

Drainage area. $--7.27 \mathrm{mi}^{2}$. Tributary to.--West Fork Kickapoo River.

Type of site.--Low-flow partial-record station.

Minimum discharge measured. $--2.42 \mathrm{ft}^{3} / \mathrm{s}$, Aug. 24, 1965.

Low-flow frequency. $-Q_{7,2}=2.6 \mathrm{ft}^{3} / \mathrm{s}, Q_{7,10}=2.1 \mathrm{ft}^{3} / \mathrm{s}$.

Basis of estimate. - Correlated with Knapp Creek near Bloomingdale using 24 discharge measurements made in the period 1961-70.

Accuracy. $-\mathrm{SE}_{7,10}=10$ percent.

05409000 West Fork Kickapoo River near Readstown, Wis.

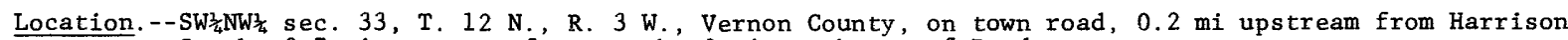
Creek, $0.7 \mathrm{mi}$ ups tream from mouth, 2 mi northeast of Readstown.

Drainage area. $--106 \mathrm{mi}^{2}$. Tributary to. --Kickapoo River.

Type of site.--Gaging station.

Period of record.--October 1938 through September 1939.

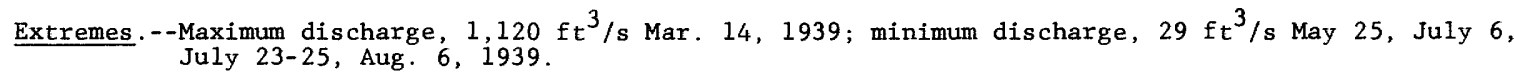

Low-flow frequency. $-Q_{7,2}=29 \mathrm{ft}^{3} / \mathrm{s}, Q_{7,10}=23 \mathrm{ft}^{3} / \mathrm{s}$.

Basis of estimate.--Correlated with Kickapoo River at La Farge using 8 discharge measurements and 4 mean daily Accuracy. $--\mathrm{SE}_{7,10}=13$ percent.

05409100 Harrison Creek near Readstown, Wis.

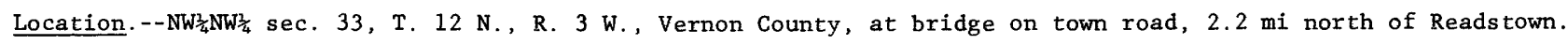
Drainage area. $--11.9 \mathrm{mi}^{2}$. Tributary to. - West Fork Kickapoo River.

Type of site.--Miscellaneous site.

Discharge measurement.--Aug. $19,1970,3.96 \mathrm{ft}^{3} / \mathrm{s}$.

05409300 Reads Creek near Readstown, Wis.

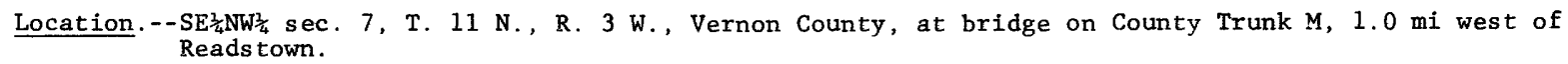
Drainage area. $--15.1 \mathrm{mi}^{2}$. Tributary to.--Kickapoo River.

Type of site.--Miscellaneous site.

Discharge measurement.--Aug. 19, 1970, $7.44 \mathrm{ft}^{3} / \mathrm{s}$. 


\section{Kickapoo River at Soldiers Grove, Wis.}

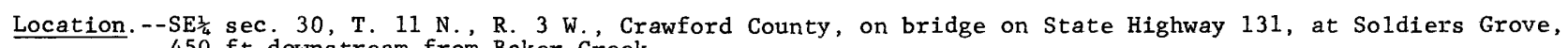
450 ft downstream from Baker Creek.

Drainage area. $--530 \mathrm{mi}^{2}$. Tributary to. - -Wisconsin River.

Type of site.--Gaging station.

Period of record.--October 1938 through September 1939.

Extremes.--Maximum discharge, 1,670 $\mathrm{ft}^{3} / \mathrm{s}$ Mar. 15, 1939; minimum discharge, $151 \mathrm{ft} / \mathrm{s}$ Jan. 22 , 1939.

Low-flow frequency. $-Q_{7,2}=160 \mathrm{ft}^{3} / \mathrm{s}, Q_{7,10}=135 \mathrm{ft}^{3} / \mathrm{s}$.

Basis of estimate.--Correlated with Kickapoo River at La Farge using 9 mean daily discharges during tl a period 1938-39.

Accuracy. $--\mathrm{SE}_{7,10}=12$ percent.

05409700 Tributary to Tainter Creek tributary near Soldiers Grove, Wis.

Location.--SW $\frac{1}{4} N W \frac{1}{4}$ sec. 32 , T. 11 N., R. 4 W., Crawford County, at bridge on County Trunk C, 5.7 mi west of Soldiers Grove.

Drainage area. $--1.11 \mathrm{mi}^{2}$.

Tributary to.--Tainter Creek tributary.

Type of site.--Miscellaneous site.

Discharge measurements.--Aug. $12,1969,0.43 \mathrm{ft}^{3} / \mathrm{s} ;$ Sept. $6,1972,0.62 \mathrm{ft}^{3} / \mathrm{s}$.

05409800 Tainter Creek near Gays Mills, Wis.

Location.--SW $\frac{1}{4} \mathrm{sec}$, 4, T. 10 N., R. 4 W., Crawford County, at bridge on County Trunk B, 3.5 mi north of Gays Mills.

Drainage area. $--38.3 \mathrm{mi}^{2}$. Tributary to. --Kickapoo River.

Type of site.--Low-flow partial-record station.

Minimum discharge measured. $--12.5 \mathrm{ft}^{3} / \mathrm{s}$, Sept. 6, 1967.

Low-flow frequency. $-\mathrm{Q}_{7,2}=12 \mathrm{ft}^{3} / \mathrm{s}, Q_{7,10}=10 \mathrm{ft}^{3} / \mathrm{s}$.

Basis of estimate. - Correlated with Kickapoo River at La Farge using 10 discharge measurements made in the period 1963-76.

Accuracy. $--\mathrm{SE}_{7,10}=17$ percent.

05409825 North Fork Nederlo Creek near Gays Mills, Wis.

Location.--SW $\frac{1}{4} \mathrm{SW}_{\frac{1}{4}} \mathrm{sec} .1, \mathrm{~T} .10 \mathrm{~N} ., \mathrm{R} .5 \mathrm{~W} ., 0.6 \mathrm{mi}$ upstream from private road bridge, $5.0 \mathrm{mi}$ northwest of Gays Mills.

Drainage area. $--1.88 \mathrm{mi}^{2}$.

Tributary to.--Tainter Creek.

Type of site.--Miscellaneous site.

Discharge measurement.--Aug. 6, 1969, $0.27 \mathrm{ft}^{3} / \mathrm{s}$. 
05409830 North Fork Nederlo Creek near Gays Mills, Wis.

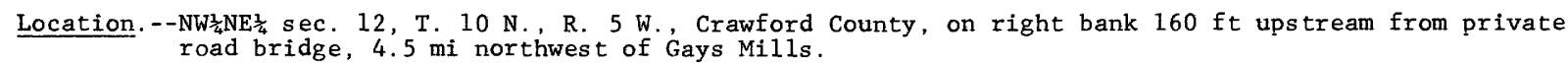

Drainage area. $--2.21 \mathrm{mi}^{2}$. Tributary to. --Tainter Creek.

Type of site.--Gaging station.

Period of record.--October 1967 through September 1975.

Average discharge. --8 years, $0.86 \mathrm{ft}^{3} / \mathrm{s}$.

Extremes.--Maximum discharge, $541 \mathrm{ft}^{3} / \mathrm{s}$ June 23, 1968; minimum discharge, $0.34 \mathrm{ft}^{3} / \mathrm{s} \mathrm{Feb.} 22,1971$.

Low-flow frequency. $-\mathrm{Q}_{7,2}=0.68 \mathrm{ft}^{3} / \mathrm{s}, \mathrm{Q}_{7,10}=0.56 \mathrm{ft}^{3} / \mathrm{s}$.

Basis of estimate.--Frequency analysis using 7-day low-flow value for 9 years of record (climatic years 1969-77).

\begin{tabular}{|lcccccc|}
\hline \multicolumn{7}{|c|}{ Duration table of daily flow } \\
Discharge, in cubic feet per second, which \\
was exceeded for indicated percent of time
\end{tabular}

Accuracy. $-\mathrm{SE}_{7,2}=3$ percent, $\mathrm{SE}_{7,10}=5$ percent.

05409842 North Fork Nederlo Creek near Gays Mills, Wis.

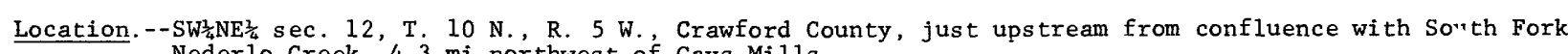
Nederlo Creek, $4.3 \mathrm{mi}$ northwest of Gays Mills.

Drainage area. $--2.31 \mathrm{mi}^{2}$. Tributary to. --Tainter Creek.

Type of site.--Miscellaneous site.

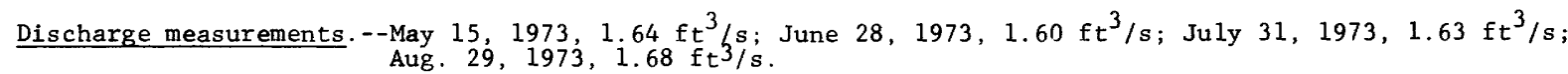

05409853 South Fork Nederlo Creek tributary near Gays Mills, Wis.

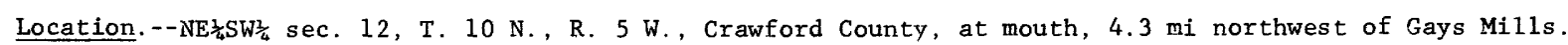
Drainage area. $--0.78 \mathrm{mi}^{2}$. Tributary to.--South Fork Nederlo Creek.

Type of site.--Miscellaneous site.

Discharge measurement.--Aug. 8, 12, 1969, $0.41 \mathrm{ft}^{3} / \mathrm{s}$.

05409855 South Fork Nederlo Creek near Gays Mills, Wis.

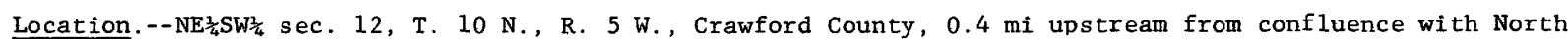
Fork Nederlo Creek, $4.4 \mathrm{mi}$ northwest of Gays Mills.

Drainage area. $--3.81 \mathrm{mi}^{2}$.

Tributary to. --Nederlo Creek.

Type of site. --Miscellaneous site.

Discharge measurement.--Aug. 12, 1969, $0.78 \mathrm{ft}^{3} / \mathrm{s}$. 
Table 1.--Low-flow characteristics for sites in the lower Wisconsin River basin--Continued

05409860 South Fork Nederlo Creek near Gays Mills, Wis.

Location.--SW $\mathrm{NEE}_{\frac{1}{4}}$ sec. 12, T. $10 \mathrm{~N}$., R. 5 W., Crawford County, $200 \mathrm{ft}$ upstream from confluence with Ncrth Fork Nederlo Creek, $4.3 \mathrm{mi}$ nor thwest of Gays Mills.

Drainage area. $--4.11 \mathrm{mi}^{2}$. Tributary to. --Neder1o Creek.

Type of site.--Gaging station.

Minimum discharge measured. $-0.76 \mathrm{ft}^{3} / \mathrm{s}$, Oct. $1-6,1967$.

Low-flow frequency. $-Q_{7,2}=0.88 \mathrm{ft}^{3} / \mathrm{s}, Q_{7,10}=0.56 \mathrm{ft}^{3} / \mathrm{s}$.

Basis of estimate. --Correlated with Nederlo Creek near Gays Mills using 12 daily discharge values made during the period 1967-76.

Accuracy.-- $-\mathrm{SE}_{7,10}=13$ percent.

Remarks.--Operated as a gaging station for a project for the period 1967-76 without publication of the records.

05409862 Nederlo Creek near Gays Mills, Wis.

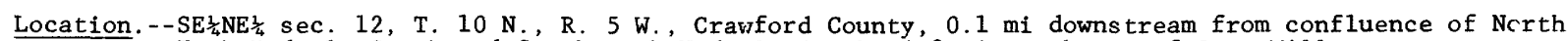
Fork Nederlo Creek and South Fork Nederlo Creek, 4.3 mi northwest of Gays Mills.

Drainage area. $--6.50 \mathrm{mi}^{2}$. Tributary to. --Tainter Creek.

Type of site.--Miscellaneous site. Discharge measurements.--Aug. $6,1969,2.01 \mathrm{ft}^{3} / \mathrm{s} ;$ Mar. $26,1973,2.76 \mathrm{ft}^{3} / \mathrm{s} ;$ May $15,1973,3.50 \mathrm{ft} / \mathrm{s}$;

Low-flow frequency. $-Q_{7,2}=1.9 \mathrm{ft}^{3} / \mathrm{s}, Q_{7,10}=1.3 \mathrm{ft}^{3} / \mathrm{s}$.

Basis of estimate.--Correlated with Nederlo Creek near Gays Mills using 4 discharge measurements.

Accuracy.--SE $7,10=19$ percent (average value for basin).

05409870 Nederlo Creek near Gays Mills, Wis.

Location.--SW $\frac{1}{4} \mathrm{NW} \frac{1}{4}$ sec. 7, T. $10 \mathrm{~N}$., R. 4 W. , Crawford County, at town road on left bank just upstream from bridge, $3.9 \mathrm{mi}$ northwest of Gays Mills.

Drainage area. $--6.70 \mathrm{mi}^{2}$. Tributary to.--Tainter Creek.

Type of site---Gaging station.

Minimum discharge measured. $--1.5 \mathrm{ft}^{3} / \mathrm{s}, \mathrm{Feb} .20-24,1968$.

Low-flow frequency. $-Q_{7,2}=2.0 \mathrm{ft}^{3} / \mathrm{s}, Q_{7,10}=1.5 \mathrm{ft}^{3} / \mathrm{s}$.

Basis of estimate.--Correlated with Nederlo Creek near Gays Mills using 13 daily discharge values.

Accuracy. $--\mathrm{SE}_{7,10}=16$ percent.

05409880 Nederlo Creek tributary near Gays Mills, Wis.

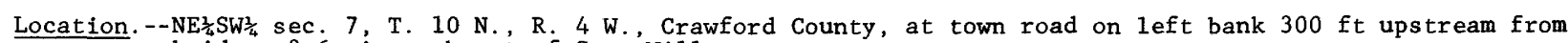
bridge, $3.6 \mathrm{mi}$ northwest of Gays Mills.

Drainage area. $--0.65 \mathrm{mi}^{2}$.

Tributary to. --Nederlo Creek.

Type of site.--Miscellaneous site.

Discharge measurement.--0ct. 17, 1967, $0.05 \mathrm{ft}^{3} / \mathrm{s}$.

05409882 Nederlo Creek tributary near Gays Mills, Wis.

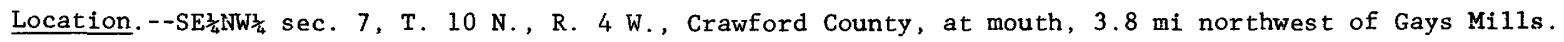

Drainage area. $--0.75 \mathrm{mi}^{2}$.

Tributary to. --Nederlo Creek.

Type of site.--Miscellaneous site.

Discharge measurement.--Aug. 6, 1969, $0.07 \mathrm{ft}^{3} / \mathrm{s}$. 
Table 1.--Low-flow characteristics for sites in the lower Wisconsin River basin--Continued

05409885 Nederlo Creek near Gays Mills, Wis.

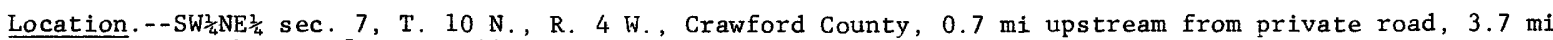
northwest of Gays Mi11s.

Drainage area. $--8.24 \mathrm{mi}^{2}$.

Tributary to.--Tainter Creek.

Type of site.--Miscellaneous site.

Discharge measurement.--Aug. 6, 1969, $2.54 \mathrm{ft}^{3} / \mathrm{s}$.

05409890 Nederlo Creek near Gays Mills, Wis.

Location. - $-\mathrm{NW} \frac{1}{4} \mathrm{NW} \frac{3}{4} \mathrm{sec} .8, \mathrm{~T} .10 \mathrm{~N}$, , R. $4 \mathrm{~W}$, Crawford County, on right bank just upstream from bridge on private road, $1.4 \mathrm{mi}$ above mouth, $3.5 \mathrm{mi}$ north of Gays Mills.

Drainage area. $--9.46 \mathrm{mi}^{2}$.

Tributary to.--Tainter Creek.

Type of site.--Gaging station.

Period of record.--October 1967 through September 1975.

Average discharge. -8 years, $4.86 \mathrm{ft}^{3} / \mathrm{s}$.

Extremes.--Maximum discharge, 2,600 $\mathrm{ft}^{3} / \mathrm{s}$ June 23, 1968; minimum discharge, $1.7 \mathrm{ft} / \mathrm{s}$ Feb. 16, 1968, result of freezeup.

Low-flow frequency. $-Q_{7,2}=3.6 \mathrm{ft}^{3} / \mathrm{s}, Q_{7,10}=2.4 \mathrm{ft}^{3} / \mathrm{s}$.

Basis of estimate.--Frequency analysis using 7-day low-flow values for 9 years (climatic years 1969-77).

\begin{tabular}{|lrrrrrrr|}
\hline \multicolumn{7}{|c|}{ Duration table of daily flow } \\
Discharge, in cubic feet per second, which \\
was exceeded for indicated percent of time \\
\hline $\begin{array}{l}\text { Percent } \\
\mathrm{ft}^{3} / \mathrm{s}\end{array}$ & 2 & 5 & 10 & 20 & 30 & 40 & 50 \\
\hline $\begin{array}{l}\text { Percent } \\
\mathrm{ft}^{3} / \mathrm{s}\end{array}$ & 11 & 6.8 & 6.0 & 5.4 & 4.8 & 4.4 & 4.2 \\
\hline
\end{tabular}

Accuracy. $--\mathrm{SE}_{7,2}=7$ percent, $\mathrm{SE}_{7,10}=10$ percent.

05409894 Nederlo Creek near Gays Mi11s, Wis.

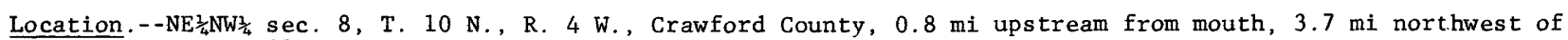
Gays Mills.

Drainage area. $--10.3 \mathrm{mi}^{2}$. Tributary to.--Tainter Creek.

Type of site.--Miscellaneous site.

Discharge measurement.--Aug. $8,1969,3.43 \mathrm{ft}^{3} / \mathrm{s}$.

05409900 Neder1o Creek at mouth near Gays Mills, Wis.

Location. - -NW $\frac{1}{4} \mathrm{SW}_{\frac{1}{4}} \mathrm{sec} .4, \mathrm{~T} .10 \mathrm{~N} ., \mathrm{R}, 4 \mathrm{~W}$. , Crawford County, at mouth, $3.7 \mathrm{mi}$ north of Gays Mills.

Drainage area. $--11.0 \mathrm{mi}^{2}$.

Tributary to.--Tainter Creek.

Type of site.--Miscellaneous site.

Discharge measurement.--Aug. $6,1969,4.59 \mathrm{ft}^{3} / \mathrm{s}$

05409999 Kickapoo River tributary at Gays Mills, Wis.

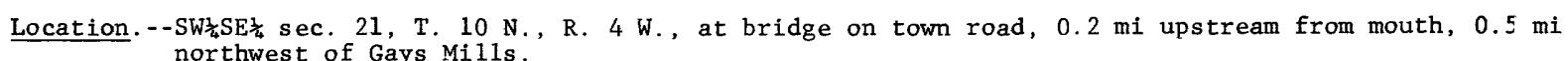

Drainage area. $--4.65 \mathrm{mi}^{2}$.

Tributary to. --Kickapoo River.

Type of site.--Miscellaneous site.

Discharge measurements.--Aug. $12,1969,2.42 \mathrm{ft}^{3} / \mathrm{s} ;$ Aug. $2,1972,2.68 \mathrm{ft}^{3} / \mathrm{s}$. 
05410000 Kickapoo River at Gays Mills, Wis .

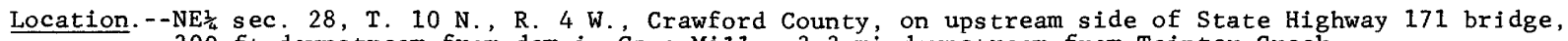
$300 \mathrm{ft}$ downstream from dam in Gays Mills, $3.3 \mathrm{mi}$ downstream from Tainter Creek.

Drainage area. $--617 \mathrm{mi}^{2}$.

Tributary to.--Wisconsin River.

Type of site.--Gaging station.

Period of record.--December 1913 to September 1934 . Monthly discharge only July to September 1934. April 1964 through September 1975.

Average discharge. - -31 years, $425 \mathrm{ft}^{3} / \mathrm{s}$.

Extremes.--Maximum discharge, $10,600 \mathrm{ft}^{3} / \mathrm{s}$ Feb. 10, 1966; minimum discharge, $48 \mathrm{ft}{ }^{3} / \mathrm{s} \mathrm{July} 27,1931$.

\begin{tabular}{|c|c|c|c|c|c|c|}
\hline \multirow[t]{2}{*}{$\begin{array}{l}\text { Period } \\
\text { of con- } \\
\text { secutive } \\
\text { days }\end{array}$} & \multicolumn{6}{|c|}{$\begin{array}{l}\text { Magnitude and frequency of annual low flow } \\
\text { Discharge, in cubic feet per second, for } \\
\text { indicated recurrence interval, in years }\end{array}$} \\
\hline & 2 & 5 & 10 & 20 & 50 & 100 \\
\hline $\begin{array}{r}7 \\
14 \\
30 \\
60 \\
90\end{array}$ & $\begin{array}{l}203 \\
216 \\
232 \\
250 \\
263\end{array}$ & $\begin{array}{l}168 \\
181 \\
196 \\
212 \\
226\end{array}$ & $\begin{array}{l}153 \\
164 \\
178 \\
193 \\
210\end{array}$ & $\begin{array}{l}142 \\
151 \\
164 \\
177 \\
197\end{array}$ & $\begin{array}{l}130 \\
137 \\
149 \\
161 \\
184\end{array}$ & $\begin{array}{l}122 \\
128 \\
139 \\
151 \\
176\end{array}$ \\
\hline
\end{tabular}

\begin{tabular}{|c|c|c|c|c|c|c|c|}
\hline \multicolumn{8}{|c|}{$\begin{array}{l}\text { Duration table of daily flow } \\
\text { Discharge, in cubic feet per second, whict } \\
\text { was exceeded for indicated percent of time }\end{array}$} \\
\hline \multirow{2}{*}{$\begin{array}{l}\text { Percent } \\
\mathrm{ft}^{3} / \mathrm{s}\end{array}$} & 2 & 5 & 10 & 20 & 30 & 40 & 50 \\
\hline & 1,700 & 980 & 630 & 470 & 400 & 350 & 320 \\
\hline \multirow{2}{*}{$\begin{array}{l}\text { Percent } \\
\mathrm{ft}^{3} / \mathrm{s}\end{array}$} & 60 & 70 & 80 & 90 & 95 & 98 & 99.9 \\
\hline & 290 & 270 & 250 & 220 & 200 & 170 & 100 \\
\hline
\end{tabular}

Accuracy. $-\mathrm{SE}_{7,2}=4$ percent, $\mathrm{SE}_{7,10}=5$ percent.

05410500 Kickapoo River at Steuben, Wis.

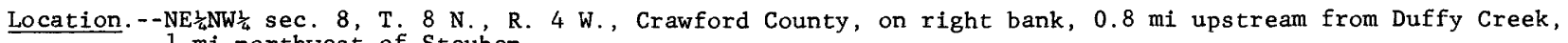
$1 \mathrm{mi}$ northwest of Steuben.

Drainage area. $--690 \mathrm{mi}^{2}$.

Tributary to.--Wisconsin River.

Type of site.--Gaging station.

Period of record.--May 1933 through September 1975.

Average discharge. -42 years, $464 \mathrm{ft}^{3} / \mathrm{s}$.

Extremes.--Maximum discharge, $10,800 \mathrm{ft}^{3} / \mathrm{s} \mathrm{Mar.} \mathrm{28,} \mathrm{1961;} \mathrm{minimum} \mathrm{discharge,} 161 \mathrm{ft} / \mathrm{s}$ Aug. 9,1936 .

\begin{tabular}{|l|rrrrrr|}
\hline $\begin{array}{l}\text { Period } \\
\text { of con- } \\
\text { secutive } \\
\text { days }\end{array}$ & $\begin{array}{l}\text { Magnitude and frequency of annual low flow } \\
\text { Discharge, in cubic feet per second, for } \\
\text { indicated recurrence interval, in years } \\
\text { ind }\end{array}$ \\
\hline & 2 & 5 & 10 & 20 & 50 & 100 \\
\cline { 2 - 7 } 7 & 248 & 207 & 190 & 177 & 164 & 156 \\
14 & 256 & 214 & 196 & 182 & 167 & 158 \\
30 & 272 & 227 & 206 & 189 & 172 & 161 \\
60 & 289 & 242 & 219 & 201 & 181 & 169 \\
90 & 306 & 253 & 228 & 208 & 187 & 173 \\
\hline
\end{tabular}

\begin{tabular}{|lrrrrrr|}
\hline \multicolumn{7}{|c|}{ Duration table of daily flow } \\
$\begin{array}{l}\text { Discharge, in cubic feet per second, which } \\
\text { was exceeded for indicated percent of time }\end{array}$ \\
$\begin{array}{l}\text { Percent } \\
\mathrm{ft}^{3} / \mathrm{s}\end{array}$ & 1,600 & 1,000 & 700 & 520 & 440 & 400 \\
\hline $\begin{array}{l}\text { Percent } \\
\mathrm{ft}^{3} / \mathrm{s}\end{array}$ & 50 & 60 & 70 & 80 & 90 & 95 \\
\hline $\begin{array}{l}\text { Percent } \\
\mathrm{ft}\end{array}{ }^{3 / \mathrm{s}}$ & 960 & 330 & 300 & 280 & 250 & 220 \\
\hline
\end{tabular}

Accuracy. $--\mathrm{SE}_{7,2}=4$ percent, $\mathrm{SE}_{7,10}=5$ percent.

05410600 Pine Creek near Steuben, Wis.

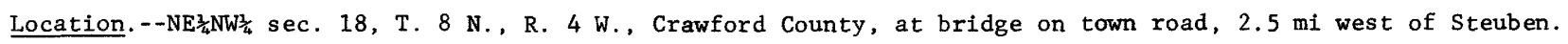
Drainage area. $--26.6 \mathrm{mi}^{2}$. Tributary to.--Kickapoo River.

Type of site.--Low-flow partial-record station.

Minimum discharge measured. $--5.63 \mathrm{ft}^{3} / \mathrm{s}$, Sept. 7, 1967.

Low-flow frequency. $--Q_{7,2}=6.4 \mathrm{ft}^{3} / \mathrm{s}, Q_{7,10}=5.1 \mathrm{ft}^{3} / \mathrm{s}$.

Basis of estimate.--Correlated with Grant River at Burton using 10 discharge measurements made in the period $1963-76$.

Accuracy. $--\mathrm{SE}_{7,10}=18$ percent. 
05410650 Plum Creek near Wauzeka, Wis.

Location. - - SW $_{4}^{\frac{1}{4}} \mathrm{NE} \frac{1}{4} \mathrm{sec} .36$, T. $8 \mathrm{~N} .$, R. $5 \mathrm{~W}$. , Crawford County, at bridge on town road, 3.4 mi northwest of
Wauzeka. Drainage area. $--16.4 \mathrm{mi}^{2}$. Tributary to.--Kickapoo River.

Type of site.--Miscellaneous site.

Discharge measurement.--Aug. 18, $1970,6.39 \mathrm{ft}^{3} / \mathrm{s}$.

05410750 Little Kickapoo Creek near Wauzeka, Wis.

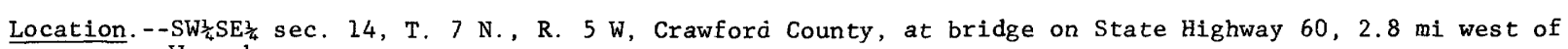
Wauzeka.

Drainage area. $--9.94 \mathrm{mi}^{2}$. Tributary to. - Wisconsin River.

Type of site.--Miscellaneous site.

Discharge measurement.--Aug. $18,1970,1.46 \mathrm{ft}^{3} / \mathrm{s}$.

05410850 Millville Creek near Wauzeka, Wis.

Location.--NW $\frac{1}{4} N_{1} \frac{1}{4}$ sec. 2, T. 6 N., R. 5 W., Grant County, at bridge on town road, 4.7 mi southwest of Wauzeka. Drainage area. $--16.3 \mathrm{mi}^{2}$. Tributary to.--Wisconsin River.

Type of site.--Miscellaneous site.

Discharge measurement.--Aug. 18, 1970,6.68 $\mathrm{ft}^{3} / \mathrm{s}$.

05410910 Dutch Hollow Creek near Wauzeka, Wis.

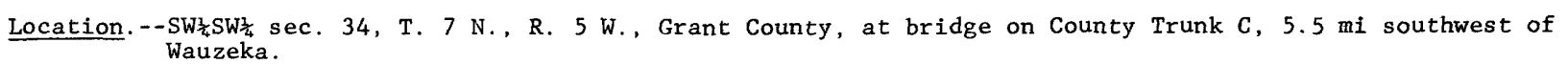
Drainage area. $--4.57 \mathrm{mi}^{2}$. Tributary to. - -Wisconsin River.

Type of site.-Miscellaneous site.

Discharge measurement.--Aug. $18,1970,0.38 \mathrm{ft}^{3} / \mathrm{s}$.

05411000 Gran Grae Creek near Wauzeka, Wis.

Location.--SW $\frac{1}{4} N E \frac{1}{4}$ sec. 32, T. 7 N., R. 5 W., Crawford County, at bridge on State Highway 60 , 6.0 mi southwest of Wauzeka.

Drainage area. $--17.1 \mathrm{mi}^{2}$. Tributary to. --Wiscons in River.

Type of site.--Low-flow partial-record station.

Minimum discharge measured. $--3.94 \mathrm{ft}^{3} / \mathrm{s}$, Sept. 7, 1967.

Low-flow frequency. $-Q_{7,2}=4.6 \mathrm{ft}^{3} / \mathrm{s}, Q_{7,10}=3.9 \mathrm{ft}^{3} / \mathrm{s}$. Basis of estimate.-- Correlated with Grant River at Burton using 11 discharge measurements made in the period

Accuracy. $--\mathrm{SE}_{7,10}=16$ percent.

05411200 Wisconsin River tributary near Bridgeport, Wis.

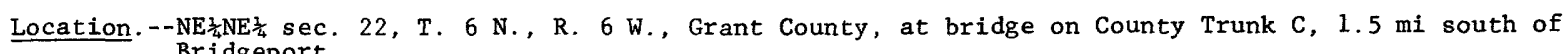
Bridgeport.

Drainage area. $--5.21 \mathrm{mi}^{2}$.

Tributary to. --Wisconsin River.

Type of site.--Miscellaneous site.

Discharge measurement.--Aug. 18, 1970, $0 \mathrm{ft}^{3} / \mathrm{s}$. 


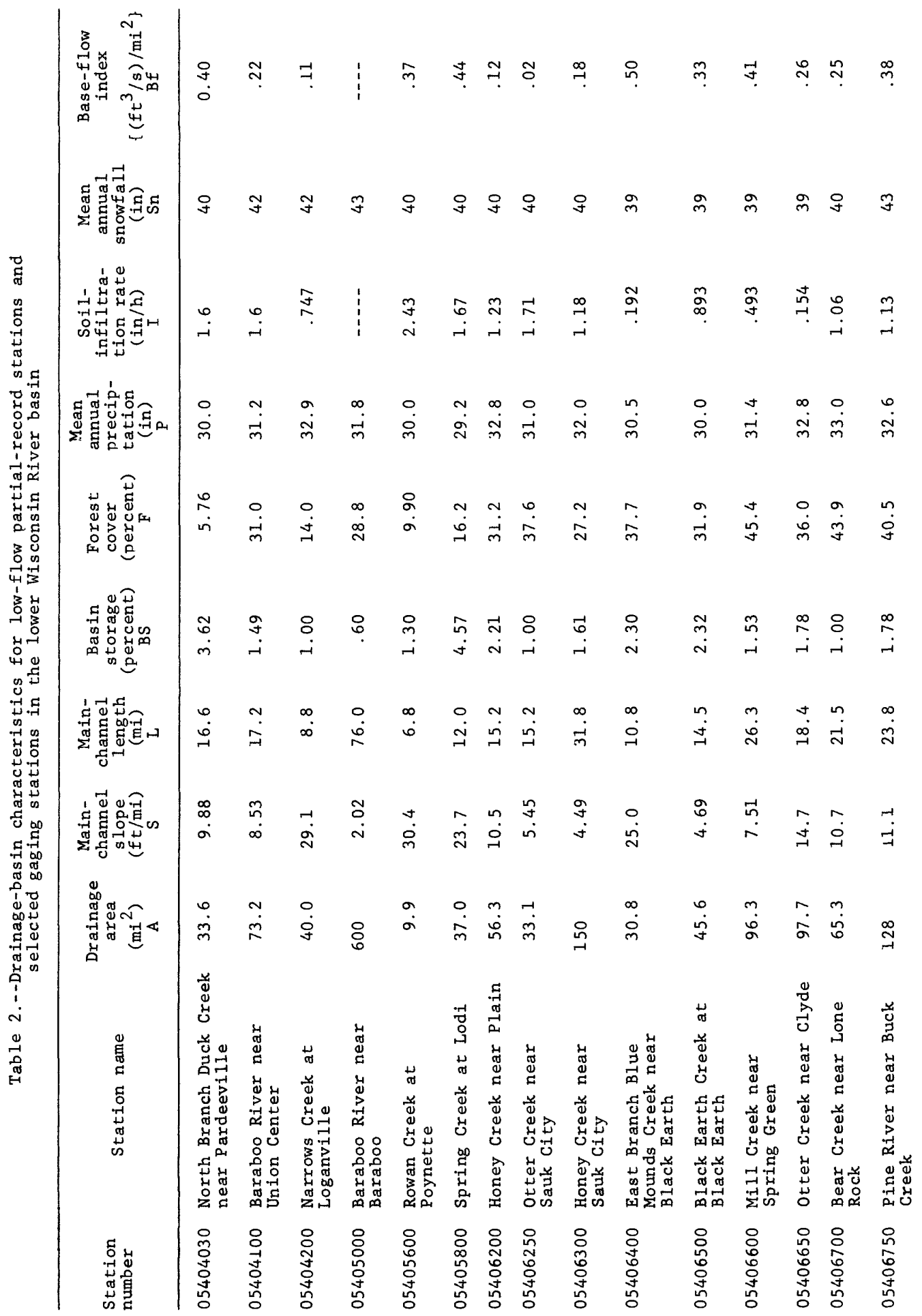




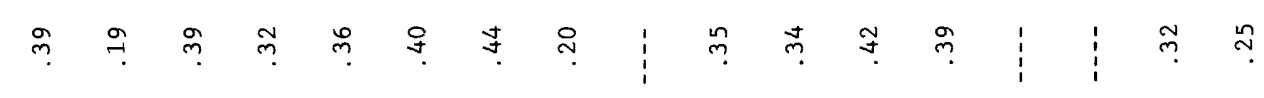

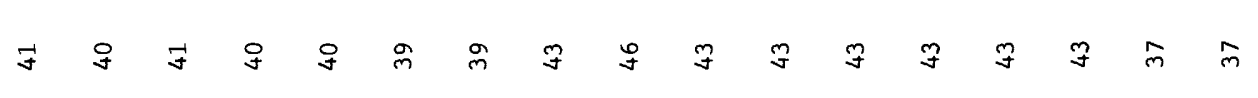

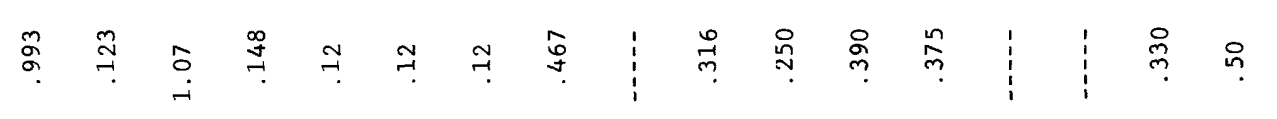

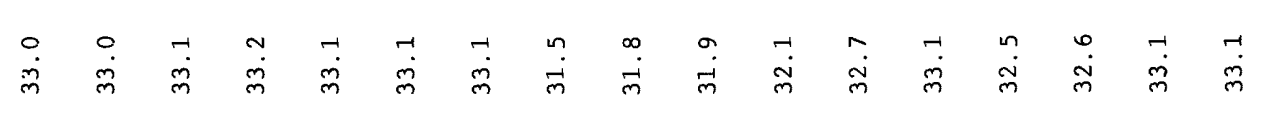

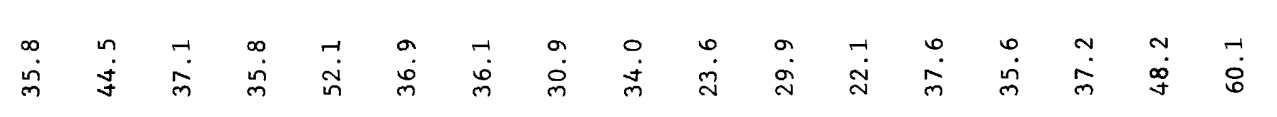

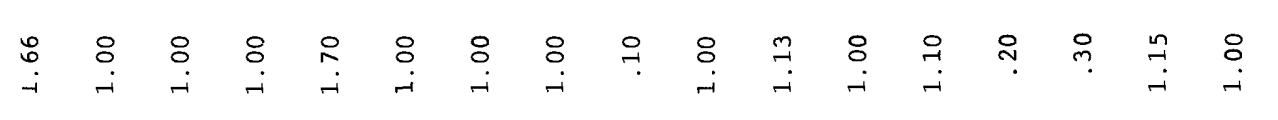

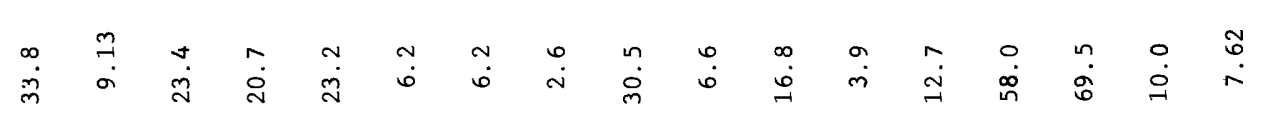

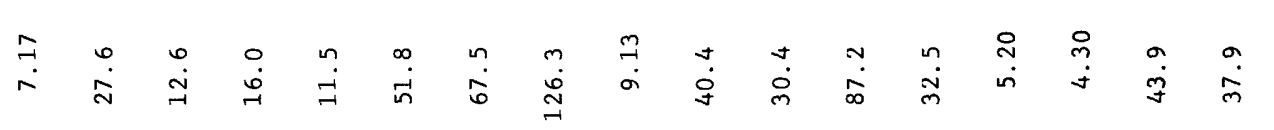

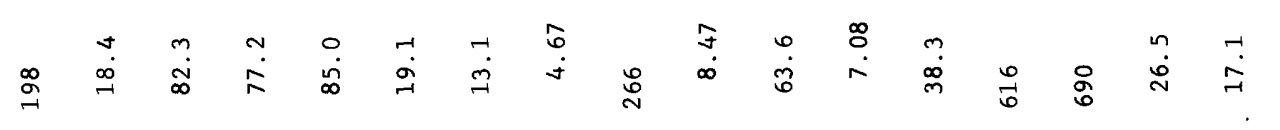

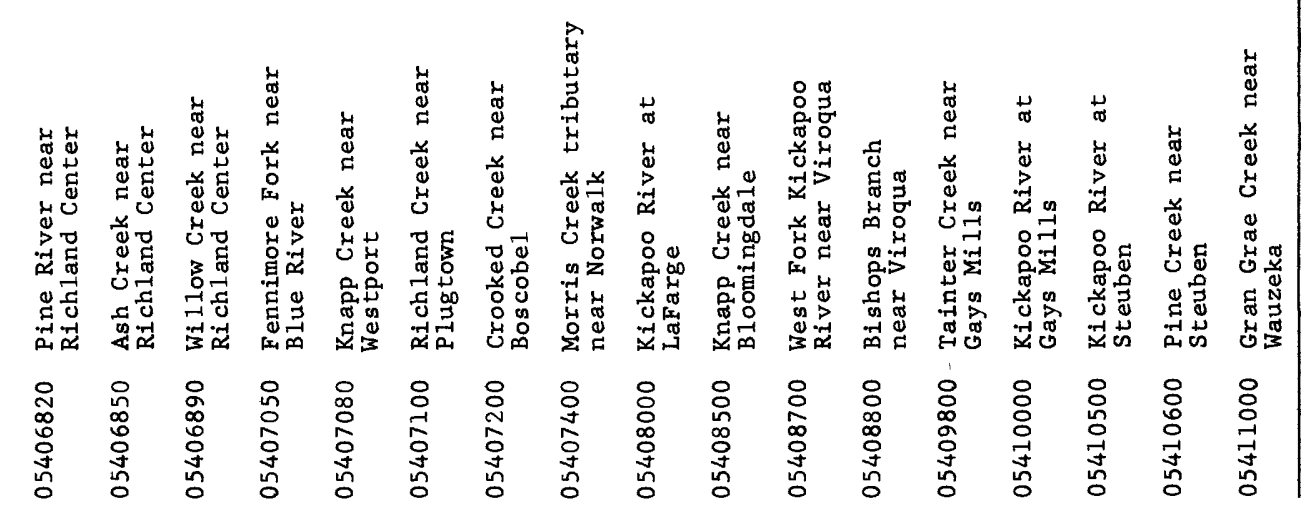




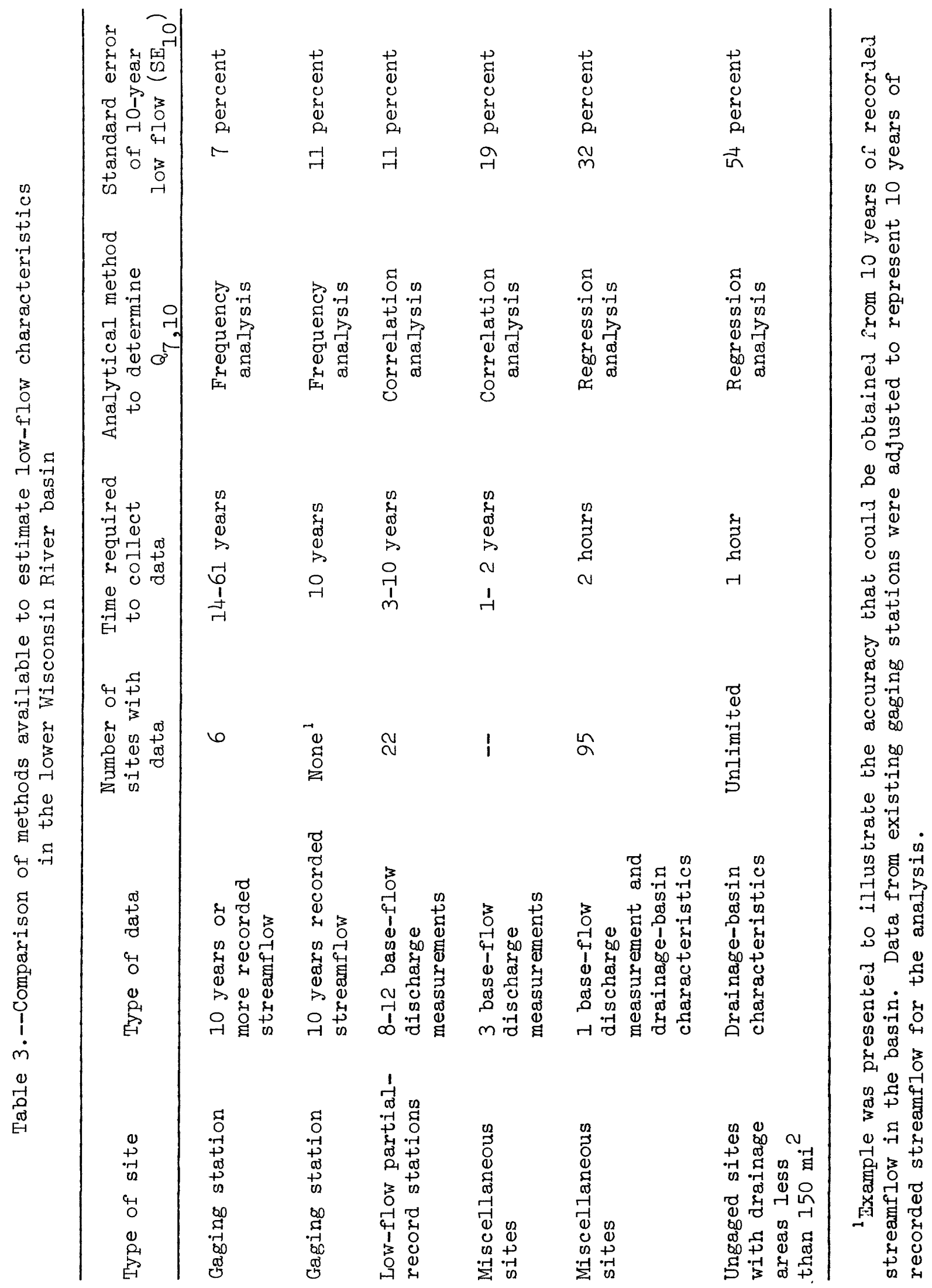

